\title{
O Índice de Conley Discreto e o Par de Matrizes de Conexão
}

\author{
Pedro Carlos Elias Ribeiro Junior
}

Orientadora: Maria do Carmo Carbinatto

Dissertação apresentada ao Instituto de Ciências Matemáticas e de Computação, da Universidade de São Paulo, como parte dos requisitos para obtenção do título de "Mestre em Ciências - Área: Matemática".

\section{USP - São Carlos}

\section{Agosto de 1999}

\footnotetext{
${ }^{1}$ Este trabalho teve suporte financeiro do CNPq e da FAPESP.
} 


\section{AGRADECIMENTOS}

Agradeço à todas as pessoas que me apoiaram durante todo este período da minha vida acadêmica, principalmente à minha família que sempre foi muito compreensiva nas horas de maiores tensões c à minha querida orientadora Maria do Carmo Carbinatto que sempre esteve ao meu lado buscando realizar o melhor trabalho possível e causou em mim 11 am amadurecimento acadêmico notável.

Agradeço também aos mens orientadores de graduação, Prof. Dr. Herminio Cassago Jr. e Profa. Dra. Sandra Maria Semenssato de Godoy, os quais me introduziram ao caminho da pesquisa acadêmica e fizeram com que eu descobrisse a beleza da Matemática.

Agradeço aos órgãos $\mathrm{CNPq}_{\text {e }} \mathrm{FAPESP}$ pelo apoio financeiro, sem o qual eu não poderia dedicar-me integralmente aos estudos.

Gostaria de agradecer a todos os professores do Departamento de Matemática que me proporcionaram grandes conhecimentos durante os cursos de Graduação e Mestrado e aos funcionários, pela infia estrutura encontrada. 


\begin{abstract}
The goal of this work is to present the connection matrix pairs for discrete Conley index.

The first part of this manuscript consists of introducing the basic concepts and results of the Conley index theory for continuous maps defined on a locally compact metric space.

We devote the second part of the work to discuss partially ordered Morse decompositions of isolated invariant sets for continnous maps and introduce the concept of connection matrix pairs. We prove the existence of such pairs for attractor-repeller decompositions of an isolated invariant set and finish with some results that illustrate how connection matrix pairs may imply the existence of connecting orbits between Morse sets.
\end{abstract}




\section{Resumo}

O objetivo deste trabalho é apresentar pares de matrizes de conexão para o índice de Conley discreto.

$\mathrm{Na}$ primcira parte do texto introduzimos os conceitos e resultados básicos da Teoria do Índice de Conley para aplicaçòes contínuas definidas em espaço métrico localmente compacto.

Dedicamos a segunda parte deste trabalho para discutir decomposições de Morse parcialmente ordenadas de conjuntos invariantes isolados para aplicações contínuas e introduzir o conceito de pares de matrizes de conexão. Provamos sua existência para decomposições atratoras-repulsoras de $u$ m conjunto invariante isolado e finalizamos com alguns resultados que ilustram como os pares de matrizes de conexão podem implicar a existência de órbitas de conexão entre conjuntos de Morse. 


\section{Sumário}

Introdução

1 O Índice de Conley Cohomológico para Aplicações Contínuas 3

1.1 Conjunto Invariante Isolado e Par-Índice . . . . . . . . . . . . . . 3

1.2 Existência de Par-índice . . . . . . . . . . . . . . 8

1.3 A Redução de Leray . . . . . . . . . . . . . . . . . . . . . . . . . . . 14

1.4 O Índice de Conley Cohomológico (Mrozek) . . . . . . . . . . . . 20

1.5 Propriedades do Índice . . . . . . . . . . . . . . . . . . . . . 21

1.6 Ponto Fixo e Ponto Periódico Hiperbólico . . . . . . . . . . . . . . . 24

2 Decomposição Atratora-repulsora $\quad 39$

2.1 Par Atrator-repulsor . . . . . . . . . . . . . . . . . . 39

2.2 Trio-índice para uma Decomposição Atratora-repulsora . . . . . . . . 45

3 Decomposição de Morse e Par de Matrizes de Conexão $\quad 59$

3.1 Decomposição de Morse e Filtração Índice . . . . . . . . . . . . . 59

3.2 Pares de Matrizes de Conexão . . . . . . . . . . . . . . . . 64

4 De volta à Decomposição Atratora-repulsora 72

4.1 Pares de Matrizes de Conexão para o Par Atrator-Repulsor . . . . . 72 
4.2 Detectando Óbitas de Conexão . . . . . . . . . . . . . . . . 75

Referências Bibliográficas 


\section{Lista de Figuras}

1.1 O par-índice $(N, L) \ldots \ldots \ldots \ldots \ldots \ldots$

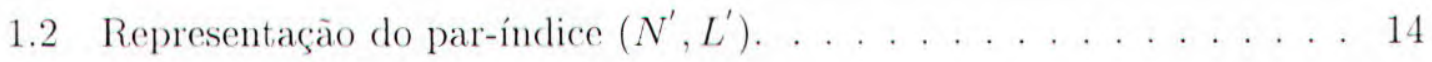

1.3 A Ferradura de Smale. . . . . . . . . . . . . . . . . . . . 34

1.4 Um par-índice para a Ferradura de Smale. . . . . . . . . . . 35

1.5 A $G$-Ferradura. . . . . . . . . . . . . . . . . . . 36

1.6 Um par-índice para a $G$-Ferradura. . . . . . . . . . . . 37

2.1 Um par atrator-repulsor. . . . . . . . . . . . . . . 56 56

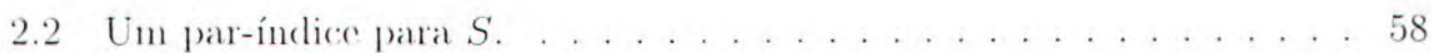




\section{Introdução}

A teoria do Índice de Conley para fluxos contínuos foi introduzida por Charles C. Conley e em [2], pode ser encontrado os fundamentos das idéias desenvolvidas por ele. O índice de Conley para sistemas dinâmicos contínuos associa, para cada conjunto invariante isolado, o tipo homotópico de un espaço pontuado. Muitas vezes, um conjunto invariante isolarlo pode ser dccomposto em conjuntos menores, chamados conjuntos de Morse, e uma ordcm parcial podc scr imposta sobre estes conjuntos. Em [5], Franzosa estenden a teoria rlo Índice de Conley para decomposições de Morse parcialmente ordenadas de um conjunto invariante isolado e apresentou o conceito de matrizes de conexão. Unia das inotivaçōcs para tal trabalho foi o desejo de obter informações a respeito a cstrutura de conjuntos definidos por uma decomposição a partir do conliecinento de índlicess de coujuntos menores dessa decomposição. Muitas aplicaçōes dos objetos definidos por Franzosa podlom scr encontrados na litcratura, porćm não as mencionarcuros aqui.

Paralclannente ì tcoria para sistcmas dinânicos contínuos, existem os sistemas dinânincos cliscretos. Naturualnentuc, surge a questão da generalização da teoria de indicc para este caso. A descrição da teoria do ínclice para o caso discreto representa a primeira parte deste trabalho. Por causa da ausência de certas propriedades que os sistemas dinâmicos contínuos possuen, o índice cliscreto apresenta uma definição mais complicarla. De fato, o índice toma a forma de um módulo graduado e um isomorfisıno distinguido. Apresentaçăo dos elementos preliminares da teoria que faremos ć baseada cm Mrozek [6].

'Temelo siclo colociaclo os aspectios baisices cla teroria, iniciannos o ponto central deste traballıo que é a construção do conccito de par de inatrizes de conexão aprensentado por Riclieson en [10]. Sua clefinição represcuta a versão discreta das matrizes de conexão definidas por Fran\%osa. A definição apressentarla toma a forma de par de matrizes; uma tendo a forma de um opcrador cobordo e outra um automorfismo 
de cocadeia. Como o índice discreto é mais complicado que o contínuo podemos esperar cune o conceito de matrizes de conexão sejan un pouco mais claborado.

Todo este traballıo cstá divido em quatro capítulos. O primeiro é dedicado em apresentar o Índice de Conley Cohomológico Discreto definido por Mrozek [6]. Para tanto clefinimos objetos tais como os conjuntos invariantes isolados e associados a cles, os pares-ínclices. Una procupação natural ć quanto a existência de um par-indlice associado a um coujunto invariante isolado c apresentamos uma prova da cxistência de par-índicc; fintalmente, temos a construção do Índice de Conley Cohonológico. Destacamos tambśn algumas propricdades que o índice apresenta. Terminamos o capítulo apresentando o cálculo do índicc para alguns exemplos.

No capítulo 2, apresentamos o conccito de decomposição atratora-repulsora para un conjunto invariante isolado. Ela representa um caso particular da decomposição de Morse, conccito que é definiclo no Capítulo 3. Também, no Capítulo 3 definimos o par de matrizes de conexão para uma decomposição de Morse de um conjunto invariante isolado. No último capítulo, provanos a existência de par de matrizes de concxão para uma decomposição atratora-rcpulsora c encerramos com resultados que mostrarn como matrizes de concxão podem implicar a existência de órbitas de concxão em sistemas dinânicos discretos. 


\section{Capítulo 1}

\section{O Índice de Conley Cohomológico para Aplicações Contínuas}

Neste capítulo apresentamos a Teoria do Índice de Conlcy para sistemas dinâmicos discrctos. Na Scção 1.1 encontrà-se a descrição das definições básicas que serão utilizaclas cin todo o traballho. Un clos conceitos importantes no qual a teoria cstá bascada é o de par-índice. Uma denonstração da cxistência de par-índice é o tópico da Seção 1.2. A construção do Ínclice de Conley Cohomológico para aplicações contínuas é feito nas Sç̧ões 1.3 c 1.4. As propriedades básicas são listadas na Seção 1.5. Finalmente, na Seção 1.6, fazcmos o cálculo do índice para alguns exemplos.

\subsection{Conjunto Invariante Isolado e Par-Índice}

Nesta seção apresentarcmos as definições básicas da teoria do Índice de Conley. A notação é bascada cm [1]. Enı todo texto $\lambda$ representará um espaço métrico localmente compacto e $f: X \rightarrow, X$ un aplicação contínua. Também, serão usadas as notações: $\mathbb{N}, \mathbb{Z}, \mathbb{Z}^{+}$para representar o conjunto dos números naturais, dos inteiros e dos inteiros positivos, respectivamcutc. As notações $\operatorname{cl}(A)$ e $\operatorname{Int}(A)$ são reservadas para o fecho e o interior de un conjuuto $A$.

Os subconjuntos de interesse no estudo de sistemas dinâmicos são os conjuntos invariantes. Sua defunição ć dacla à seguir.

Definiçāo 1.1. Dizemos que $S \subset X$ Xé um conjunto invariante se $f(S)=S$. 
Definição 1.2. Seja $N \subset X$. Definimos três subconjuntos de $N$ :

I. $\operatorname{Inv}^{-}(N, f):=\left\{x \in N \mid \exists\left\{x_{n}\right\}_{-\infty}^{n=0} \subset N\right.$ tal que $x_{n+1}=f\left(x_{n}\right)$ c $\left.x_{0}=x\right\}$,

2. $\operatorname{Inv}^{+}(N, f):=\left\{x \in N \mid \exists\left\{x_{n}\right\}_{n=0}^{\infty} \subset N\right.$ tal quc $x_{n+1}=f\left(x_{n}\right)$ c $\left.x_{0}=x\right\}$,

3. $\operatorname{Inv}(N, f):=\left\{x \in N \mid \exists\left\{x_{n}\right\}_{-\infty}^{\infty} \subset N\right.$ tal que $x_{n+1}=f\left(x_{n}\right)$ e $\left.x_{0}=x\right\}$.

Ilustramos as definições acima com um exemplo simples.

Exemplo 1.3. Seja $f: \mathbb{R}^{2} \rightarrow \mathbb{R}^{2}$ uma aplicação linear dada por $f(x, y)=\left(2 x, \frac{1}{2} y\right)$. Considere $N=[-2,2] \times[-2,2]$. A aplicação $f$ ć um homeomorfismo e $f^{-1}(x, y)=$ $\left(\frac{1}{2} x, 2 y\right)=\left(2^{-1} x,\left(\frac{1}{2}\right)^{-1} y\right)$. Mais ainda, se $n \in \mathbb{Z}$, cntão $f^{n}(x, y)=\left(2^{n} x, \frac{1}{2^{n}} y\right)$. Note que $\left\|f^{n}(x, y)\right\|=\sqrt{2^{2 n} x+2^{-2 n} y}$. Com isso, é fácil ver que se $x \neq 0$,

$$
\left\|f^{n}(x, y)\right\| \rightarrow \infty, \text { quando } n \rightarrow \infty
$$

Se $y \neq 0$,

$$
\left\|f^{-n}(x, y)\right\| \rightarrow \infty, \text { quando } n \rightarrow \infty
$$

Vamos calcular $\operatorname{Inv}(N, f), \operatorname{Inv}^{-}(N, f) \mathrm{c} \mathrm{Inv}^{+}(N, f)$.

Se $(z, 0) \in[-2,2] \times\{0\}$, então a sequência $z_{n}=f^{-n}(z, 0)$ é tal que $\left\{z_{n}\right\}_{-\infty}^{n=0}$ está inteiramente conticla em $N$. Por $(1.1)$, concluimos que $\operatorname{Inv}^{-}(N, f)=[-2,2] \times\{0\}$. Analogamente, $\operatorname{Inv}^{+}(N, f)=\{0\} \times[-2,2]$. Por (1.1) e (1.2), para todo $\left(z_{1}, z_{2}\right) \neq$ $(0,0)$, temos que, ou $f^{-n}\left(z_{1}, z_{2}\right) \rightarrow \infty$, on $f^{n}\left(z_{1}, z_{2}\right) \rightarrow \infty$, quando $n \rightarrow \infty$. Logo, $\operatorname{Inv}(N, f)=\{(0,0)\}$.

Os próximos três resultados apresentam propriedades dos conjuntos definidos acima.

Proposição 1.4. O conjunuto $\operatorname{Inv}(N, f)$ é imıvariante.

DemonstraÇÃo. Tome $x \in \operatorname{Inv}(N, f)$. Logo, existe sequência $\left\{x_{n}\right\}_{-\infty}^{\infty} \subset N$ tal que $f\left(x_{n}\right)=x_{n+1}$ c $x_{0}=x$. Considerc a sequência $\left\{y_{n}\right\}_{-\infty}^{\infty} \subset N$ dada por $y_{n}=x_{n+1}$. Porticunto,

$$
f\left(y_{n}\right)=f\left(x_{n+1}\right)=x_{n+2}=y_{n+1} \text { e } y_{0}=x_{1}=f(x)
$$


Logo, $f(x) \in \operatorname{Inv}(N, f)$. Oı seja, $f(\operatorname{Inv}(N, f)) \subset \operatorname{Inv}(N, f)$.

Agora considere $y \in \operatorname{Inv}(N, f)$. Logo, existe sequência $\left\{y_{n}\right\}_{-\infty}^{\infty} \subset N$ tal que $f\left(y_{n}\right)=y_{n+1}$ e $y_{0}=y$. Portauto, $y=f\left(y_{-1}\right)$. Resta mostrar que $y_{-1} \in \operatorname{Inv}(N, f)$. Para isto basta considerar a sequência $\left\{z_{n}\right\}_{-\infty}^{\infty} \subset N$ dada por $z_{n}=y_{n-1}$. Portanto, $f\left(z_{n}\right)=f\left(y_{n-1}\right)=y_{n}=z_{n+1}$ e $x_{0}=y_{-1}$. Logo, $y_{-1} \in \operatorname{Inv}(N, f)$. O que implica que $y \in f(\operatorname{Inv}(N, f))$. E' concluinıos a clemonstração.

Proposição 1.5. O conjunto $\operatorname{Inv}(N, f)$ é maximal, isto é, se $Y \subset N$ é um canjunto invariante, então $Y \subset \operatorname{Inv}(N, f)$.

DemonstraçÃo. Seja $x \in Y$. Como $Y$ é un conjunto invariante temos que $f^{n}(x) \in Y$, prara toclo $n \in \mathbb{N}$. Fm purticunlar, $f^{n}(x) \in N$, para torlo $n \in \mathbb{N}$. So $n \in \mathbb{N}$, rlefinn $x_{n}:=f^{n}(x)$. Assim,

$$
f\left(x_{n}\right)=f\left(f^{n}(x)\right)=f^{n+1}(x)=x_{n+1} .
$$

Alćm disso, existe $\vec{x} \in Y \subset N$ tal que $f(\vec{x})=x$. Defina $x_{-1}:=\vec{x}$. Então, existe $x_{-1} \in N$ tal que $f\left(x_{-1}\right)=x$. Um simples argumento de indução nos permitc construir $x_{-n} \in N$, com $n=-1,-2, \ldots$ tal que $f\left(x_{n}\right)=x_{n+1}$. A sequência $\left\{x_{n}\right\}_{-\infty}^{\infty} \subset N$ ć tal que $f\left(x_{n}\right)=x_{n+1}$ c $x_{0}=x$. Portanto, $x \in \operatorname{Inv}(N, f)$.

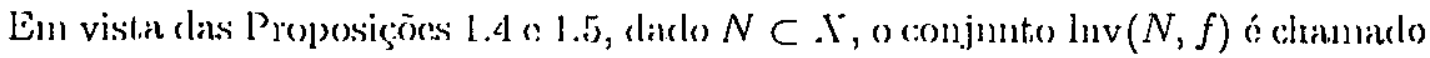
de conjunto invariante maximal.

Observação 1.6. 1. Os conjuntos $\operatorname{Inv}(N, f)=\operatorname{Inv}^{-}(N, f) \cap \operatorname{Inv}^{+}(N, f)$ também são invariantes.

2. Usaremos a notiação mais simplificada $\operatorname{Inv}(N)$ ao invés de $\operatorname{Inv}(N, f)$ quando isto não causar confusão com relação a que aplicação $f$ estamos considerando.

Proposição 1.7. Se $N \subset X^{-}$é compacto, então as conjuntos $\operatorname{Inv}^{-}(N), \operatorname{Inv}^{+}(N) e$ $\operatorname{Inv}(N)$ são subconjuntas fechados de $N$. Em particular, são compactos.

DemonstraÇão. Primeiramente mostraremos que $\operatorname{Inv}(N)$ é um conjunto fechado. Considcre $x \in \operatorname{cl}(\operatorname{Inv}(N))$. Logo, existc uma sequência $\left\{y_{n}\right\}_{n=0}^{\infty} \subset \operatorname{Inv}(N)$ tal que $y_{n} \rightarrow x$, quando $n \rightarrow \infty$. Fixe $m \in \mathbb{Z}^{+}$. Como $f^{m}$ é uma aplicação contínua, temos 
que $f^{m}\left(y_{n}\right) \rightarrow f^{m}(x)$, quando $n \rightarrow \infty$. Como $y_{n} \in \operatorname{Inv}(N)$, então $f^{m}\left(y_{n}\right) \in N$. Portanto, $f^{m}(x) \in N$. Defina $x_{m}:=f^{m}(x)$.

Mostraremos que existe $x_{m}$, com $m=-1,-2, \ldots$ tal que $f\left(x_{m}\right)=x_{m+1}$. Para cada $n \in \mathbb{N}$, temos que $y_{n} \in \operatorname{Inv}(N)$. Logo, existe uma sequência $\left\{z_{-1}^{n}\right\}_{n=-\infty}^{\infty} \subset N$ tal que $f\left(z_{-1}^{n}\right)=y_{n}$. A sequência $\left\{z_{-1}^{n}\right\}_{n=-\infty}^{\infty}$ está contida $\mathrm{cm} N$. Logo, existe uma subsequência $\left\{z_{-1}^{n_{k}}\right\}_{k=0}^{\infty}$ convergente. Seja $\bar{z}$ o limite desta subsequência. A continuidade da aplicação $f$ e a unicidade de limites implicam que $f(\bar{z})=x$. Defina $x_{-1}:=\ddot{z}$. Utilizando indução produzimos uma sequência $\left\{x_{n}\right\}_{-\infty}^{n=0}$ com as propriedades desejaclas.

Assim, $\left\{x_{n}\right\}_{-\infty}^{\infty}$ é uma sequêucia en $N$ tal que $f\left(x_{n}\right)=x_{n+1}$ e $x_{0}=x$. Portanto, $x \in \operatorname{Inv}(N)$. Sendo $\operatorname{Inv}(N)$ um subconjunto fechado de $N$, temos que $\operatorname{Inv}(N)$ é um conjuuto compacto.

Aualogamente, provamos as mesmas propriedades para $\operatorname{Inv}^{-}(N) \operatorname{e~Inv}^{+}(N)$.

A seguir apresentaremos o primciro ingredientc importante na teoria do Índice dic Conley.

Definição 1.8. Dizemos que $S \subset X$ é um conjunto invariante isolado se existe $N \subset$ $X$ compacto tal que $S=\operatorname{Inv}(N) \subset \operatorname{Int}(N)$. O conjunto $N$ é chamado vizinhança isolante de $S$.

Usando a Proposição 1.7 concluinıs gue torlo conjuntoto invariante isolado é um conjunto compacto.

A cada conjunto invariante isolado é associado um par de conjuntos compactos. Esse par é o segundo elenconto findamental da teoria do Índice de Conley e sua definição é dada a seguir.

Definição 1.9. Seja $S$ um conjunto invariante isolado. Um par de subconjuntos compactos $(N, L)$ é um par-índice para $S$ com relação a $f$ se satisfaz às seguintes condições:

1. $S=\operatorname{Inv}(\operatorname{cl}(N \backslash L)) \subset \operatorname{Int}(N \backslash L)$,

2. $f(L) \cap N \subset L$, 
3. $f(N \backslash L) \subset N$.

Observação 1.10. 1. A condição 2 de par-índice é equivalente a dizer que $L$ é positivamente invariante com rolação a $N$.

2. Se $(N, L)$ é um par-índice para o conjunto invariante isolado $S$ tal que

$$
\operatorname{cl}(N \backslash L) \cap \operatorname{cl}(f(L) \backslash N)=\emptyset
$$

cutão ć chamado de par-índice regular.

Exemplo 1.11. Tome $\int$ c $N$ cono no Excmplo 1.3. Assim, temos que $S=$ $\operatorname{Inv}(N)=\{(0,0)\} \subset \operatorname{Int}(N)$. Logo, $N$ é vizinhança isolante para $\{(0,0)\}$. Considere o conjunto $L=([-2,-1] \times[-2,2]) \cup([1,2] \times[-2,2])$. A representação geométrica do par $(N, L)$ ć dada na Figura 1.1.

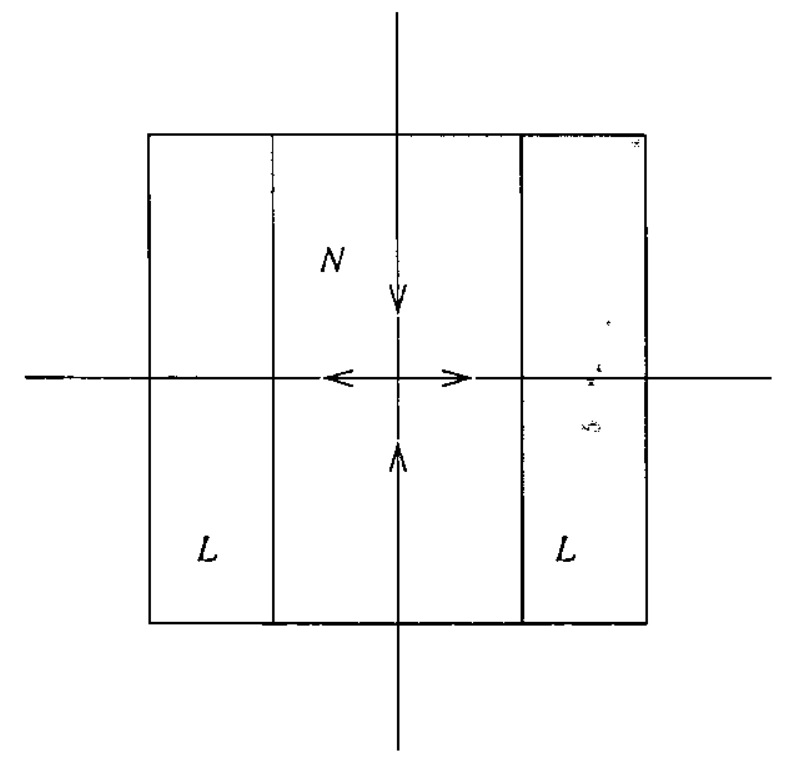

Figura 1.1: O par-índice $(N, L)$.

Vaulos mostiar que $(N, L)$ ć un par-índice para $S$. Note que $\operatorname{cl}(N \backslash L)=$ $[-1,1] \times[-2,2]$. Desta mancira, tomos $\{(0,0)\}=\operatorname{Inv}(\operatorname{cl}(N \backslash L)) \subset \operatorname{Int}(N)$.

Para mostrar que $L$ ć positivannente invariante com relação a $N$ devemos calcular $f(L) \cap N$. Tenos que, $f(L) \cap N=(\{-2\} \times[-1,1]) \cup(\{2\} \times[-1,1]) \subset N$.

Finalunente, $N \backslash L=(-1,1) \times[-2,2]$. Logo, $f(N \backslash L)=(-2,2) \times[-1,1]$. E est.c está contido cu $N$. Portianto, o par $(N, L)$ ć um par-ínclice para $\{(0,0)\}$. 
Mais ainda, verifica-sc que $\operatorname{cl}(N \backslash L) \cap \operatorname{cl}(f(L) \backslash N)=\emptyset$. Portanto, o par $(N, L)$ ć um par-índice regular para $\{(0,0)\}$.

A proposição abaixo apresenta umı caracterização alternativa para a propriedade 3 da definição de par-índice.

Proposição 1.12. Seja $(N, L)$ um porr de subconjuntos de $X$. Então $f(N \backslash L) \subset N$, sc, e somente se, para lodo $x \in N$ tal que $f(x) \notin N$, implica $x \in L$.

DemonstraçÃo. Seja $x \in N$ tal que $f(x) \notin N$ e suponlia que $x \notin L$. Logo, $x \in N \backslash L$. Portanto, $f(x) \in N$, o que é uma contradição.

Para finalizar a demonstração, scja $x \in N$ tal que $x \notin L$. Qucremos inostrar que $f(x) \in N$. Suponhar (une $f(x) \notin N$. Logo, $x$ deve ser un elemento de $L$, o que ć uma contradição.

Observaçāo 1.13. Em vista da proposição acima, o conjunto $L$ é comumente cliamado conjunto de saida.

\subsection{Existência de Par-índice}

Daclo um conjunto invarianta isolaclo $S$ a perguntia que se fà ć cono construir um par-ínclice para $S$. O objetivo desta seção é apresentar o teorema da existência de par-ínclice provado por Franks c Richıcson [4]. Iniciamos esta seção definindo o conjunto saida imediato.

Definição 1.14. Seja $N$ vizinhanç̧ isolante para o conjunto invariante isolado $S$. $O$ conjunto $N^{-}:=N \backslash f^{-1}(\operatorname{Int}(N))$ é chamado de conjunto saída imediato.

Observação 1.15. Note que $N^{-}=\{x \in N \mid f(x) \notin \operatorname{Int}(N)\}$.

Definição 1.16. Sejam $S$ um conjunto invariante isolado e $N$ sua vizinhança isolante. Se

$$
N \cap f(N) \cap f^{-1}(N) \subset \operatorname{Int}(N),
$$

então $N$ é chamado bloco isolante.

Nem tocla vi\%inlaunca isolantes s um bloco isolante. De fatoo 
Exemplo 1.17. Considcre a aplicação linear $f: \mathbb{R}^{2} \rightarrow \mathbb{R}^{2}$ definida por $f(x, y)=$ $(y, 2 x)$. Scja $N=[-2,2] \times[-1,1]$. Note (luc $\operatorname{Iuv}(N)=\{(0,0)\}$. Logo, $N$ é uma viziılıaıça isolante para $\{(0,0)\}$. Teınos que $f^{-1}(z, w)=(w / 2, z)$ é a inversa da aplicação $f$. Note que o ponto $(0,1)$ está lla fronteira de $N$ e pertence a interseç̧ão $N \cap f(N) \cap f^{-1}(N)$. Portianto, $N \cap f(N) \cap f^{-1}(N) \not \subset \operatorname{Int}(N)$. Ou seja, $N$ não é bloco isolante.

A existência de bloco isolante implica ua existência de par-índice regular.

Proposiçāo 1.18. Sejam $S$ um conjunto invariante isolado e $N$ sua vizinhança isolante. Se $N$ for um bloco isolante, então $\left(N, N^{-}\right)$é par-indice regular para $S$.

DemonstraÇÃo. Deveınos mostrar que as condições 1, 2, c 3 da Definição 1.9 e a condição apresentada na Observação 1.10 estão satisfeitas.

Mostremos que $S=\operatorname{Inv}\left(\operatorname{cl}\left(N \backslash N^{-}\right)\right)$. Colıo $N \backslash N^{-} \subset N$, temos que $\operatorname{cl}\left(N \backslash N^{-}\right) \subset$ N. Logo,

$$
\operatorname{Iuv}\left(\operatorname{cl}\left(N \backslash N^{-}\right)\right) \subset \operatorname{Iuv}(N)=S
$$

Seja $x \in S$. Como $N$ é vizinhança isolantc para $S$, temos que $f(x) \in \operatorname{Int}(N)$. Logo, $x \notin N^{-}$. Portanto, $S \subset N \backslash N^{-}$. O que implica

$$
S \subset \operatorname{Inv}\left(\operatorname{cl}\left(N \backslash N^{-}\right)\right)
$$

Seja $x \in S$. Logo, existe $r_{1}>0$ tal que $B\left(x, r_{1}\right) \subset N$. Como $f(x) \in \operatorname{Int}(N)$, cxis te $\varepsilon_{0}>0$ tal que $B\left(f(x), \varepsilon_{0}\right) \subset N$. A continuidade de $f$ implica que existe $\delta>0$ tal que $f(B(x, \delta)) \subset B\left(f(x), \varepsilon_{0}\right)$. Defina

$$
r:=\min \left\{\delta, r_{l}\right\}
$$

Temos que

$$
B(x, r) \subset N
$$

Além disso, se

$$
y \in B(x, r)
$$

entĩo

$$
\int(y) \in \int(B(x, r)) \subset B\left(f(x), \varepsilon_{0}\right) .
$$


Porlianto, para toclo $y \in B(n, v)$, temos cue $f(y) \in \operatorname{Inh}(N)$. Logo, $y \notin N^{-}$. Assin,

$$
B(x, r) \subset N \backslash N^{-} .
$$

Com isso, a condição 1 da Definição 1.9 cstá verificada.

Passemos à prova da condição 2 para o par $\left(N, N^{-}\right)$. Seja $y \in f\left(N^{-}\right) \cap N$, e suponha que $y \notin N^{-}$. Logo, $y=f(x) \operatorname{com} x \in N^{-}$e $f(y) \in \operatorname{Int}(N)$. Como $N$ ć bloco isolante, temos que $y \in \operatorname{Int}(N)$. Portanto, $x \in f^{-1}(\operatorname{Int}(N))$, o que é uma contradiç̧ão. Ou seja, $f\left(N^{-}\right) \cap N \subset N^{-}$. A condição 3 da Definção 1.9 também é facilmente demonstrada c deixamos os cletalhes para o leitor.

Para encerrar a demonstração, mostremos que

$$
\left.\operatorname{cl}\left(N \backslash N^{-}\right) \cap \operatorname{cl}\left(f\left(N^{-}\right) \backslash N\right)\right)=\emptyset .
$$

Suponlia que existc $\left.y \in \mathrm{cl}\left(N \backslash N^{-}\right) \cap \mathrm{cl}\left(f\left(N^{-}\right) \backslash N\right)\right)$. Vamos mostrar que isto implica que $y \in N \cap f^{-1}(N) \cap f(N)$. De fato, como $y \in \operatorname{cl}\left(N \backslash N^{-}\right)$, existe uma sequência $\left\{y_{n}\right\}_{n=0}^{\infty} \subset N \backslash N^{-}$tal que $y_{n} \rightarrow y$, (llando $n \rightarrow \infty$. Como $N$ é compacto, temos que $y \in N$.

Como $y_{n} \in N \backslash N^{-}$, temos que $f\left(y_{n}\right) \in \operatorname{Int}(N)$. A continuidade de $f$ e a compacilarle de $N$ implicam que $f(y) \in N$. Por (1.3), temos que $y \in \operatorname{cl}\left(f\left(N^{-}\right) \backslash N\right)$. Portanto, existe una sequência $\left\{z_{n}\right\}_{n=0}^{\infty} \subset f\left(N^{-}\right) \backslash N$ tal que $z_{n} \rightarrow y$, quando $n \rightarrow \infty$. Além disso, para cada $n \in \mathbb{N}$, existe $w_{n} \in N^{-}$tal que $z_{n}=f\left(w_{n}\right)$. Sem perda de generalidacle, podemos supor quc $\left\{w_{n}\right\}$ ć uma sequencia convergente em $N$. Seja $w$ sen limte. A continuidade da aplicação $f$ implica que $f(w)=y$. Portanto, $y \in f(N)$.

Como $N$ ć bloco isolante, seguc quıc $y \in \operatorname{Int}(N)$. Por outro lado, como a sequência $\left\{z_{n}\right\}_{n=0}^{\infty}$ definida acina converge para $y$, cxiste $n_{0} \in \mathbb{N}$ tal que $z_{n} \in \operatorname{Int}(N)$, para todo $n \geq n_{0}$. Porém, $z_{n} \in f\left(N^{-}\right) \backslash N$. Estce fato gera uma contradição. Assim, a intcrsecção dos conjuntos cm (1.3) deve ser vazia.

En vista da proposição acima, torna-sc importantc saber como determinar ou até mesmo como constrir blocos isolantes. A sequência de definições e resultados a seguir inıplicará na cxistência de blocos isolantes.

Definição 1.19. Sejam $S$ um conjunto invariante isolado e $N$ sua vizinhança iso- 
lante. Para cada $\varepsilon>0$, o conjunto

$$
\begin{aligned}
C_{\varepsilon}(N, S):= & \left\{x \in N \mid \exists\left\{x_{k_{k}}\right\}_{-k}^{k} \subset N, \text { com } x_{-k}, x_{k} \in S, x_{0}=x\right. \\
& \text { c clist } \left.\left(f\left(x_{n}\right), x_{n+1}\right)<\varepsilon \text { para todo } n=-k, \ldots, k-1\right\}
\end{aligned}
$$

é chamado vizinhança $\varepsilon$-cacleia de $S$ em relação a $N$.

Definição 1.20. Sejam $S$ um conjunto invariante isalado e $N$ sua vizinhança isolante. Para cada $m \in \mathbb{N}$, definimos

$$
\begin{aligned}
& \operatorname{Inv}^{m}(N):=\left\{x \in N \mid \exists\left\{x_{n}\right\}_{n=m}^{-m} \subset N \text { com } f\left(x_{n}\right)=x_{n+1}\right. \\
& \left.\quad \text { para } n=-m,-m+1, \ldots, m-1 \text { e } x_{0}=x\right\} .
\end{aligned}
$$

O lema enunciado a seguir nos ajudará na demonstração de existência de $\varepsilon$ carleias.

Lema 1.21. Sejam $S$ um conjunto invariante isolodo e $N$ sua vizinhança isolante. Para toda $V$ vizinhança de $S \mathrm{~cm} N$, existe $m_{0} \in \mathbb{N}$ tal que $\operatorname{Inv}^{m_{0}} \subset V$.

Demonstração. Suponha que para todo $m \in \mathbb{N}$ tenhamos $\operatorname{Inv}^{m}(N) \not \subset V$. Assim, para cada $m \in \mathbb{N}$, cxistc $x_{m} \in \operatorname{Inv}^{m}(N)$ tal que $x_{m} \notin V$. Como $V$ é vizinhança de $S$ em $N$, tenıos que $x_{m} \in N$. Tonıando uma subsequência, se necessário, podemos supor que $x_{m} \rightarrow x$, quando $n \rightarrow \infty, \operatorname{com} x \in N$.

Note que para todo $k \leq m$, temos que $f^{k}\left(x_{m}\right) \in N$. Devido a continuidade da aplicação $f$ e a compacidade de $N$, podemos concluir que $f^{k}(x) \in N$, para todo $k \leq m$. Defina $x_{k}:=f^{k}(x)$.

Como $x_{m} \in \operatorname{Inv}^{m}(N)$, temos que cxiste uma sequência $\left\{z_{j}^{m}\right\}_{j=-m}^{m} \subset N$ tal que $f\left(z_{j}^{m}\right)=z_{j+1}^{m}$, para $j=-m,-m+1, \ldots, m-10 z_{0}^{m}=x_{m}$. Considerando a sequência $\left\{z_{-1}^{m}\right\}_{m=0}^{\infty} \subset N$, tomando unna subsequência, se necessário, podemos supor que $z_{-1}^{m} \rightarrow x_{-1}$, com $x_{-1} \in N$. A continuidade cla aplicação $f$ e unicidade de limites nos garante que $f\left(x_{-1}\right)=x$. Procedenclo de mancira auláloga, cricontramos uma sequência $\left\{w_{n}\right\}_{-\infty}^{\infty} \subset N$ tal (que $f\left(w_{n}\right)=w_{n+1}$ e $w_{0}=x$. Portanto, $x \in S \subset V$, o que é uma contradição.

A proposição a seguir ć o resultarlo que nos garante a existência de $\varepsilon$-cadeias. 
Proposição 1.22. Seja $S$ um conjunto invwriantc isolado e $N$ sua vizinhança isolankle. Para qualquer vizimhanca $V$ de $S$ em $N$, existe $\varepsilon_{0}>0$ tal que $C_{\varepsilon_{o}}(N, S) \subset V$.

Demonstração. Tome $V \subset N$ uma vizinhança tal que $S \subset V$. Pelo lema anterior, cxistc $m_{0} \in \mathbb{N}$ tal que $\operatorname{Inv}^{m_{0}}(N) \subset V$.

Suponha que para todo $\varepsilon>0$, existe $x(\varepsilon) \in C_{\varepsilon}(N, S)$ tal que $x \notin V$. Logo, para cada $\varepsilon>0$, existe uma sequêucia

$$
\begin{gathered}
\left\{x_{n}(\varepsilon)\right\}_{n=-m(\varepsilon)}^{m(\varepsilon)} \subset N, \text { com } x_{0}(\varepsilon)=x(\varepsilon), x_{-m(\varepsilon)}, x_{m(\varepsilon)} \in S \\
\text { c } \operatorname{dist}\left(f\left(x_{n}(\varepsilon)\right), x_{n+1}(\varepsilon)\right)<\varepsilon \text {, para } n=-m(\varepsilon),-m(\varepsilon)+1, \ldots, m(\varepsilon)-1 .
\end{gathered}
$$

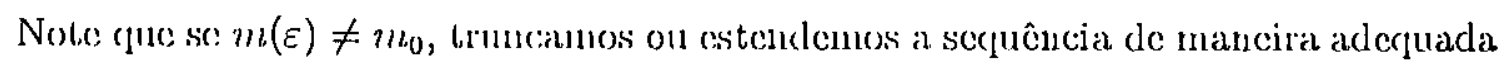
de modo que a sequência $\left\{x_{n}(\varepsilon)\right\}_{n=-m_{0}}^{m_{0}} \subset N$ satisfaça às condiçōes: $x_{0}(\varepsilon)=x(\varepsilon)$, $x_{-m_{0}}(\varepsilon), x_{m_{0}}(\varepsilon) \in S$ e $\operatorname{dist}\left(f\left(x_{n}(\varepsilon)\right), x_{n+1}(\varepsilon)\right)<\varepsilon, \operatorname{com} n=-m_{0},-m_{0}+1, \ldots, m_{0}-$ 1 .

Escolha uma sequência $\left\{\varepsilon_{i}\right\}_{i=1}^{\infty}$ tal que $\varepsilon_{i}>0$ o $\varepsilon_{i} \rightarrow 0$, quando $i \rightarrow \infty$. Como para cada $n=-m_{0}, \ldots, m_{0}$ e para cada $i \in \mathbb{N}$ temos que $x_{n}\left(\varepsilon_{i}\right) \in N$, a compacidade dc $N$ nos garante que porlcmos assumir que a sequência $\left\{x_{n}\left(\varepsilon_{i}\right)\right\}_{i=0}^{\infty}$ converge para um elemento de $N$. Seja $x_{n}$ o linite desta sequência. Facilmente conclui-se que $f\left(x_{n}\right)=x_{n+1}$, para todo $n=-m_{0}, \ldots, m_{0}$. Portanto, $x \in \operatorname{Inv}^{m_{0}}(N) \subset V$. O que é uına contradição.

Finalmente temos a existência de par-índice regular para um conjunto invariante isolado.

Proposição 1.23. Toda vizinhança isolante de um conjunto invariante isolado contérn um bloco isolante. Em particular, se $N$ é uma vizinlıança isolante para $S$, então, para qualquer $\varepsilon>0$ suficicntemente pequeno, $\operatorname{cl}\left(C_{\varepsilon}(N, S)\right)$ é um bloco isolante.

Demonstração. Seja $V$ vizinhanç̧a de $S$ em $N$. Tome $\varepsilon_{0}>0$ como na Proposição 1.22. Logo, $C_{\varepsilon_{0}}(N, S) \subset V$. Defina $B:=\operatorname{cl}\left(C_{\varepsilon_{0}}(N, S)\right)$. Suponha que $B$ não seja bloco isolante, ou scja, cxistc $y \in B \cap f(B) \cap f^{-1}(B)$ tal quc $y \notin B$. Como $y \in f(B)$, existe $x \in B$ tal que $y=f(x)$. Portanto, $y \in f^{-1}(B)$ e $f^{2}(x) \in B$.

Coino $f$ é uma aplicação contínua em $X$, existe $\delta>0$ tal que se $\operatorname{dist}(x, y)<\delta$, cntão $\operatorname{clist}(f(x), f(y))<\varepsilon_{0}$. Como $x \in B$, cxistc $\bar{x} \in C_{\varepsilon_{0}}(N, S)$ tal que $\operatorname{dist}(x, \bar{x})<\delta$. 
Além disso, existc una sequência $\left\{x_{n}\right\}_{n=-k}^{k} \subset N$ tal que $x_{0}=\bar{x}, x_{-k}, x_{k} \in S \mathrm{e}$ $\operatorname{dist}\left(f\left(x_{n}\right), f\left(x_{n+1}\right)\right)<\varepsilon_{0}$, para $n=-k,-k+1, \ldots, k-1$.

Tambón temos cue $f^{2}(x) \in B$. Cono anterionnente, cxistc $\bar{y} \in C_{\varepsilon_{0}}(N, S)$ tal que $\operatorname{dist}\left(f^{2}(x), \bar{y}\right)<\varepsilon_{0}$ e existe uma sequência $\left\{y_{n}\right\}_{n=-m}^{m} \subset N$ tal que $y_{0}=\bar{y}, y_{-m}$, $y_{n} \in S$ e $\operatorname{dist}\left(f\left(y_{n}\right), f\left(y_{n+1}\right)\right)<\varepsilon_{0}$, para $n=-m,-m+1, \ldots, m-1$. Considere a sequência

$$
x_{-k}, x_{-k+1}, \ldots, x_{-1}, x_{0}, f(x), y_{0}, \ldots, y_{m}
$$

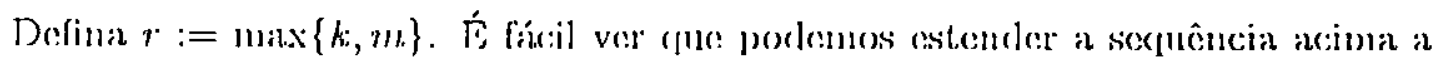
fim de obter uma sequência $\left\{z_{n}\right\}_{n=-r-1}^{r+1} \subset N$, ondc

$$
z_{-1}=x_{0}, z_{-2}=x_{-1}, \ldots, z_{-r-1}=x_{-r}, z_{0}=f(x), z_{1}=y_{0}, \ldots, z_{r+1}=y_{r}
$$

Note que $\operatorname{dist}\left(f\left(z_{n}\right), z_{n+1}\right)<\varepsilon_{0}$, para $n=-r-1, \ldots, r$. Ainda, $z_{-r-1}, z_{r} \in S \mathrm{e}$ $z_{0}=f(x)$. Logo, $y=f(x) \in C_{\varepsilon_{0}}(N, S)$. O đule ć una contradição.

Darlo $11 \mathrm{~m}$ conjunto invariante isolado $S$ estiamos interessados cm definir um invariante para $S$ baseado em um par-índice associado a $S$. Par o caso de fluxos contínuos o tipo homotópico do par-índice consistc em um invariante (Conley [2]). Para o caso de sistemas dinâmicos discretos, o tipo homotópico do par-índice (e portanto, a teoria de homologia ou cohomologia) não representa um invariante. $O$ exemplo abaixo ilustra este fato.

Exemplo 1.24. Considere a aplicação $f: \mathbb{R}^{2} \rightarrow \mathbb{R}^{2}$ tal que $f(x, y)=\left(2 x, \frac{1}{2} y\right)$. Mostramos no Exemplo 1.11 que $(N, L)$ é par-índice para $S=\{(0,0)\}$. Na Figura 1.1 temos uma representação geométrica do par $(N, L)$. Agora considere o par $N^{\prime}=([-2,2] \times[-2,2]) \cup([-1,1] \times[3,4])$ e $L^{\prime}=L$. (Ver Figura 1.2). Então o par $\left(N^{\prime}, L^{\prime}\right)$ taunbéun ó parr-íudice para $S$.

Mas o tipo homotópico do espaço topológico pontuado $\left(N \backslash L_{\imath}[L]\right)$ é diferente do tipo homotópico de $\left(N^{\prime} \backslash L^{\prime},\left[L^{\prime}\right]\right)$. E, ć claro que este fato também acarreta que os grupos do colomologia relativa $H^{*}(N, L) \propto H^{*}\left(N^{\prime}, L^{\prime}\right)$ são clistintos. De fato,

$$
H^{\prime \prime}(N, L)= \begin{cases}\mathbb{Z}, & \text { sı } q=1, \\ 0, & \text { caso contrário. }\end{cases}
$$

Porcin no caso do par $\left(N^{\prime}, L^{\prime}\right)$ temos cule:

$$
H^{\prime \prime}\left(N^{\prime}, L^{\prime}\right)= \begin{cases}\mathbb{Z}, & \text { se } q=0,1, \\ 0, & \text { caso contrário, }\end{cases}
$$




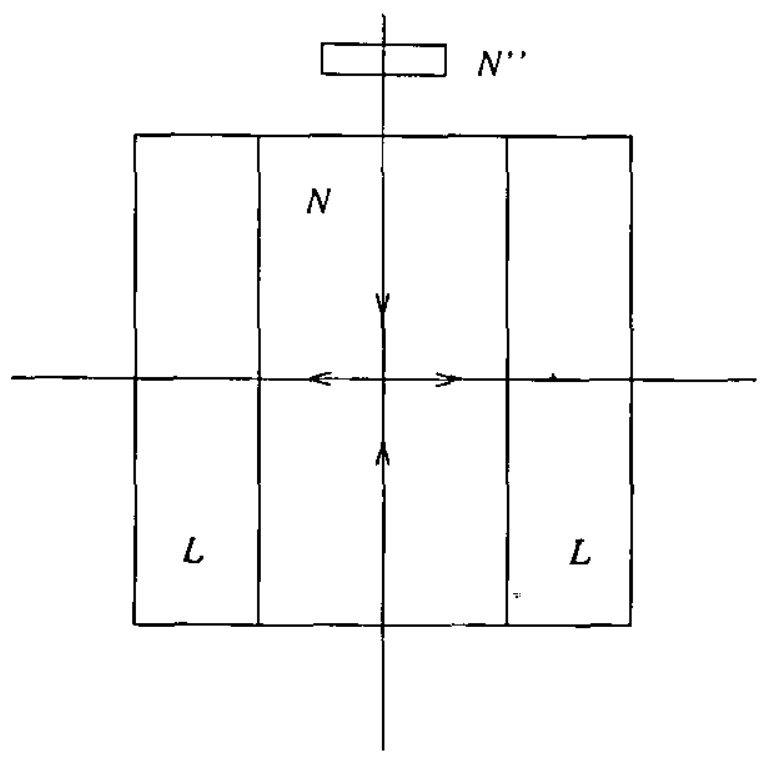

Figura 1.2: Representação do par-índice $\left(N^{\prime}, L^{\prime}\right)$.

ou seja, $H^{*}(N \backslash L,[L])$ ou $H^{*}(N, L)$ não define um invariante para $S$.

A construção do Índice de Conlcy que apresentaremos na seção 1.4 é devido a Mrozek [6]. Na próxima scção descreveremos a Redução de Leray que foi a ferramenta utilizada por Mrozek para sua construção do invariante.

\subsection{A Redução de Leray}

Ncstia seção descrevemos a Redução do Leray e destiacamos algumas propriedades que são importantes na construção do Índico do Conlcy Colıomológico e na demonstração das propriedades desse invariante.

Inicianos definindo a categoria dos Endomorfismos, que será denotada por $\mathcal{E}$. Um objeto desta categoria ć um par $(F, f)$, onde $F$ é um módulo sobre um anel e $f: F \rightarrow F$ é um endomorfismo.

Dados dois objetos $(F, f)$ e $(E, e)$ em $\mathcal{E}$, um morfismo entre $(F, f)$ e $(E, e)$ é uma aplicação $\varphi: F \rightarrow E$ tal que $\varphi \circ f=e \circ \varphi$.

Definição 1.25. Sejann $F$ um módulo solnre um anel e $f: F \rightarrow F$ um endomorfis- 
mo. O núclco generalizado de $f$ é dado por

$$
\operatorname{gkcr}(f):=\left\{x \in F \mid f^{n}(x)=0 \text {, para algum } n \in \mathbb{N}\right\}
$$

É fácil ver que

Lema 1.26. Sejain $F$ um módulo, $f: F \rightarrow F$ um endomorfismo e $n \in \mathbb{N}$. Se $x \in \operatorname{ker}\left(h^{n}\right)$, então parn torla $m \geq n$, temos que $x \in \operatorname{ker}\left(f^{m}\right)$.

Demonstração. Scja $x \in \operatorname{kcr}\left(f^{n}\right)$. Logo, $f^{n}(x)=0$. Coino $m$ é um inteiro positivo maior ou igual a $n$, cxiste $p \in \mathbb{N}$ tal que $m=n+p$. Assim

$$
f^{\prime \prime}(x)=f^{\prime \prime}\left(f^{n}(x)\right)=f^{p}(0)=0
$$

Iogo, $: x \in \operatorname{kor}\left(f^{m}\right)$

Como $f(\operatorname{gker}(f)) \subset \operatorname{gker}(f)$, faz sentido definir a aplicação induzida $f^{\prime}$

$$
f^{\prime}:(F / \operatorname{gkcr}(f)) \rightarrow(F / \operatorname{gker}(f))
$$

dacla por $f^{\prime}([x])=[f(x)]$. Utilizaremos a notação $L M(F, f)$ para denotar o par $\left(f / \operatorname{gker}(f), f^{\prime}\right)$. Note que $L M(F, f)$ é um objeto de $\mathcal{E}$.

Observaçāo 1.27. Observc que $f^{\prime}$ ć um mollomorfismo.

Sc $\varphi$ ć um morfismo cntre $(E, e)$ c $(F, f)$, verifica-se facilmente que $\varphi$ (gker $(e)) \subset$ gkcr $(f)$. Assim faz sentido definir a aplicação $\varphi^{\prime}:(E / \operatorname{gker}(e)) \rightarrow(F / \operatorname{gker}(f))$ dada por $\varphi^{\prime}([x])=[\varphi(x)]$. A aplicação $\varphi^{\prime}$ faz o sliagrama a seguir comutar.

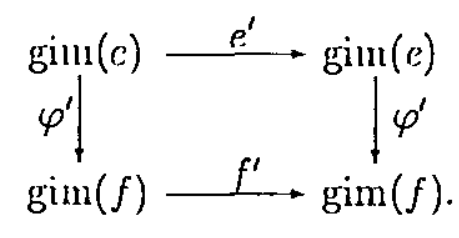

Denotaremos $\varphi^{\prime}$ por $L M(\varphi)$. Observe que $L M(\varphi)$ é um morfismo entre $L M(F, f)$ e $L M(E, e)$. Também, $L M(\varphi)$ é um monomorfismo. 
Definição 1.28. Sejam $F$ um módulo sobre um anel e $f: F \rightarrow F$ um monomorfismo. O conjunto

$$
\operatorname{gim}(f):=\left\{x \in F \mid \exists\left\{x_{n}\right\}_{n=0}^{\infty} \subset F \text { lall que } f^{n}\left(x_{n}\right)=x \text { pura todo } n \in \mathbb{N}\right\},
$$

é cliamado inagem generalizadla de f.

Conno $f(\operatorname{gin}(f)) \subset \operatorname{gim}(f)$, faz scutido considerar a aplicação

$$
f^{\prime \prime}: \operatorname{gin}(f) \rightarrow \operatorname{gin}(f)
$$

dacla por $f^{\prime \prime}(x)=f(x)$. Notemos que $f^{\prime \prime}$ é um monomorfismo e que também será um epinorfismo, sendo então um isomorfismo. Vamos denotar o par $\left(\operatorname{gim}(f), f^{\prime \prime}\right)$ por $L I(F, f)$. Observe que, novamente, esta construção produz um objeto em $\mathcal{E}$.

Suponlia que $\varphi:(E, e) \rightarrow(F, f)$ seja um morfismo entre $(E, e)$ e $(F, f)$, com $e$ e $f$ monomorfismos. Podemos assim definir a aplicação $\varphi^{\prime \prime}: \operatorname{gim}(e) \rightarrow \operatorname{gim}(f)$, dada por $\varphi^{\prime \prime}(x)=\varphi(x)$. Além disso, o diagrama a seguir é comutativo:

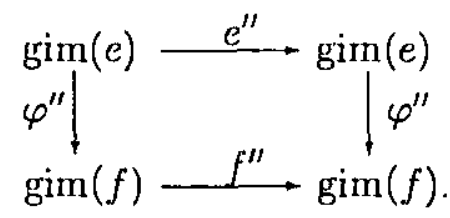

Portanto, $\varphi^{\prime \prime}$ é um morfismo entre $L I(F, f)$ c $L I(E, e)$. Iremos denotá-lo por $L I(\varphi)$.

Daulo $(F, f)$ unu objelo su $\varepsilon$, o par $\mathcal{L}(F, f):=L I(L M(F, f))$ ć chamado a Redução de Leray de $(F, f)$. Se $\varphi$ ć unn morfismo entre $(F, f)$ e $(E, e)$, denotamos por $\mathcal{L}(\varphi)$ o morfismo $\operatorname{LI}(\operatorname{LM}(\varphi))$.

Esta é a ferramenta que usaremos para a construção do Índice de Conley Cohomológico Discreto. Encerrarenıos a scção demonstrando algumas propriedades da Redução de Leray.

Proposição 1.29. Sejann $E$ e $F$ mótlulos solve unn ancl e $: E \rightarrow E$ ef $f F \rightarrow F$

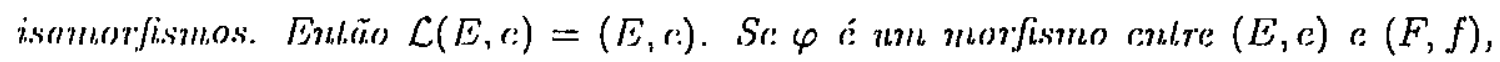
então $\mathcal{L}(\varphi)=\varphi$. 
Demonstração. Como $c$ e $f$ são isomorfismos, temos que

$$
\operatorname{gker}(e)=E, \operatorname{gkcr}(f)=F, \operatorname{gim}(e)=E \text { e } \operatorname{gim}(f)=F .
$$

Logo,

$$
\operatorname{gim}(E / \operatorname{gker}(e))=E \text { e } \operatorname{gim}(F / \operatorname{gker}(f))=F .
$$

Assim, ao aplicarmos a Reclução de Leray, obtcmos cue $c^{\prime \prime}(x)=e(x)$ e $f^{\prime \prime}(x)=$ $f(x)$. Logo, a aplicaçã̃o $\varphi$ "` $\varphi$. Assim $\mathcal{L}(\varphi)=\varphi$.

A scguir vamos demonstrar cono a Redução de Leray se comporta com relação a.o produto cartesiano.

Definiçāo 1.30. Sejum $\left(E, e^{\prime}\right) e(F, f)$ dois objetos em $\mathcal{E}$. Definimos o produto cartesiano $E \times F$, como o natural e o endomorfismo $(e \times f): E \times F \rightarrow E \times F$, dada por $(e \times f)(x, y)=(e(x), f(y))$.

Observaçāo 1.31. Sejam e $: E \rightarrow E$ e $f: f \rightarrow F$ dois endomorfismos de módulos, entĩo $(e \times f)^{n}=\left(e^{n}, f^{n}\right)$.

A fim de facilitar a demonstração da Proposição 1.34 enunciamos alguns lemas auxiliares.

Lema 1.32. Sejam $(E, e)$ e $(F, f)$ dois objetos en $\mathcal{E}$. Então

1. $\operatorname{gker}(e \times f)=\operatorname{gker}(e) \times \operatorname{gkcr}(f)$,

2. $E \times F / \operatorname{gkcr}(e \times f)=E / \operatorname{gkcr}(e) \times f / \operatorname{gkcr}(f)$,

3. $(c \times f)=e^{\prime} \times f^{\prime} e$

1. $\operatorname{LM}(E \times F, c \times f)=\operatorname{LM}(E, c) \times \operatorname{LN}(F, f)$.

Demonstração. Scja $z=(x, y)$, com $x \in E$ e $y \in F$, tal que $z \in \operatorname{gker}(e \times f)$. Logo, cxiste, $n_{0} \in \mathbb{N}$ tal clue $(e \times f)^{n_{0}}(z)=0$. Ou scja, $\left(e^{n_{0}}(x), f^{n_{0}}(y)\right)=(0,0)$. Ternos que $x \in \operatorname{gker}(e) \mathrm{c} y \in \operatorname{gkcr}(f)$. Portanto, $z=(x, y) \in \operatorname{gker}(e) \times \operatorname{gker}(f)$.

Agora tome $(x, y) \in \operatorname{gker}(e) \times \operatorname{gker}(f)$. Logo, existem $n_{1}$ e $n_{2}$, tais que $e^{n_{1}}(x)=0$ e $f^{n_{2}}(y)=0$. Usando o Lcina 1.26, verifica-sc facilmente quc existe $n_{0} \in \mathbb{N}$ tal que $\left(e^{n_{0}}(x), f^{n_{0}}(y)\right)=(e, f)^{n_{0}}(x, y)=(0,0)$. Ou seja, $(x, y) \in \operatorname{gker}(e \times f)$. 
Para a verificação do item 2, considere $[z]=[(x, y)]$, com $x \in E$ e $y \in F$ tal que $[z] \in(E \times F) / \operatorname{gkcr}(e \times f)$. Sc $w \in[z]$, então $w=(x, y)+\bar{w}$, com $\bar{w} \in \operatorname{gker}(e \times f)$.

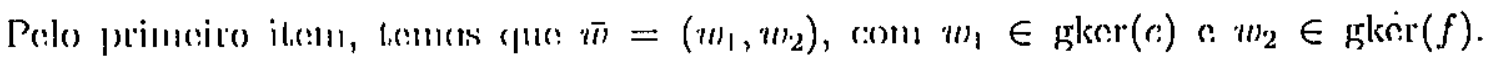
Assim

$$
u=(x, y)+\left(w_{1}, w_{2}\right) \in[x] \times[y] \in E / \operatorname{gker}(e) \times F / \operatorname{gkcr}(f)
$$

De ınodo análogo prova-sc a outra inclusão. Para provar 3 , notemos que os itcıs antcriores nos garantenı que a aplicaçāo $(e \times f)^{\prime}: E / \operatorname{gker}(e) \times F / \operatorname{gker}(f) \rightarrow$ $E / \operatorname{gkcr}(e) \times F / \operatorname{gker}(f)$, é dada por $(e \times f)^{\prime}(x, y)=[e(x), f(y)]$. Ou seja,

$$
(c \times f)^{\prime}=e^{\prime} \times f^{\prime} .
$$

A denınstração do ńltinıo itenı do lema é uma questão da aplicação dos itcns anteriores.

Lema 1.33. Sejam $(E, e)$ e $(F, f)$ dois objetos em $\mathcal{E}$. Então

1. $\operatorname{gim}(e \times f)=\operatorname{gim}(e) \times \operatorname{gim}(f)$

"). $(r \times f)^{\prime \prime}=r^{\prime \prime} \times f^{\prime \prime}$ :

3. $L I(E \times F, e \times f)=L I(E, e) \times L I(F, f)$.

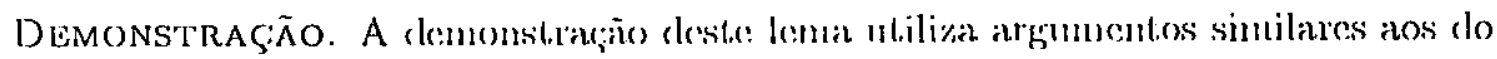
Lemtil 1.32 .

Proposição 1.34. Sejum $E$ e $F$ móllulos sobre um anel, e $: E \rightarrow E$ ef $: F \rightarrow F$ endomorfismos. Então,

$$
\mathcal{L}((E, e) \times(F, f))=\mathcal{L}(E, e) \times \mathcal{L}(F, f)
$$

Demonstração. Aplicando o Lemla 1.32 e em seguida o Lema 1.33, obtemos o resultado desejado.

Proposiçāo 1.35. Seja $\mathbb{O}$ um objeto ern $\mathcal{E}$, onde $E=\{0\}$ e o endomorfismo é o nulo. Entõo $\mathcal{L}(\mathbb{O})=\mathbb{O}$.

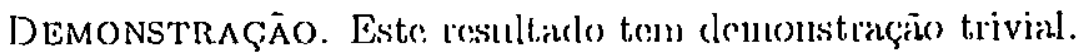


Definição 1.36. Scjum $(E, c)$ a $(F, f)$ doiss objollas cm $\mathcal{E}, \varphi$ um morfismo entre $(E, e) e(F, f)$ e $\psi$ um morfisnı entre $(F, f)$ e $(E, e)$. Diremos que os objetos $(E, e)$ $e(F, f)$ são ligados pelos morfismos $\varphi$ e $\psi$ se o diagrama

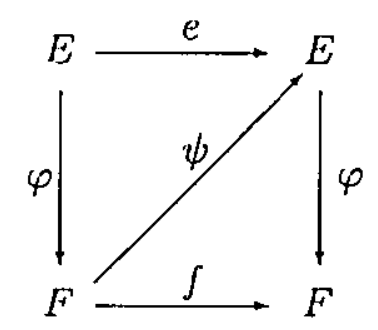

é comutativo.

Daddos $(E, e)$ e $(F, f)$ dois ol,jetos em $\mathcal{E}$ liganlos pelos morfismos $\varphi$ e $\psi$, temos que o diagrama

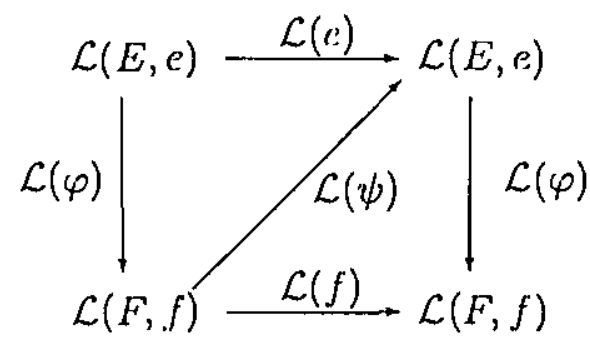

também é comutativo.

Proposição 1.37. $\operatorname{Sejam}(E, e) e(F, f)$ dois objelos em $\mathcal{E}$, ligados pelos morfismos $\varphi$ e $\psi$. Então $\mathcal{L}(\varphi)$ e $\mathcal{L}(\psi)$ são isonorfismos. Em particular, $\mathcal{L}(E, e)$ e $\mathcal{L}(F, f)$ são isomorfos.

A demonstração desta proposição poder ser encontrada em Mrozek [6]. Iremos finalizar com o resultado que diz que a Redução de Leray é exata. Mais precisamente.

Proposição 1.38. Seja $\left(E_{1}, c_{1}\right) \rightarrow\left(E_{2}, e_{2}\right) \rightarrow\left(E_{3}, e_{3}\right)$ uma sequência exata. Então $\mathcal{L}\left(E_{1}, e_{1}\right) \rightarrow \mathcal{L}\left(E_{2}, e_{2}\right) \rightarrow \mathcal{L}\left(E_{3}, e_{3}\right)$ é uma sequência exata. 


\subsection{O Índice de Conley Cohomológico (Mrozek)}

Finalmente chegamos a clescrição cla construção clo Índice de Conley Cohomológico apresentado por Mrozck. A exposição a seguir ó bascacla em [1].

Recordemos que $S$ representa mul conjunto invariante isolado e $(N, L)$ um parindice para $S$.

Lema 1.39. Se $(N, L)$ é un par-índice para um conjunto invariante isolado $S$, então

1. $(N, L) \subset(N \cup f(L), L \cup f(L))$.

2. $A$ inclusão

$$
i:(N, L) \hookrightarrow(N \cup f(L), L \cup f(L))
$$

induz um isomorfismo na colomalogia de Alcaander-Spainer.

DemonstraÇÃo. A inclusĩo enı (1) ć facilmentc provada. A afirmativa em (2) decorre da propricdade da excisão for te da teoria de colomologia. Ver [6], Teorema 5 do capílulo 6.5 para mais detallics.

Agora considere a aplicação dacla por

$$
f_{N I_{\alpha}}^{\#}=f^{\#} \circ\left(i_{N I_{1}}^{\#}\right)^{-1}: C^{*}(N, L) \rightarrow C^{*}(N, L)
$$

onde

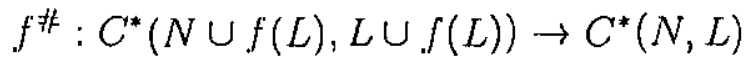

ć a induzida colonológica da f no nívol de cocadcia. Passando à cohomologia obtemos a aplicação

$$
f_{N L}^{*}: H^{*}(N, L) \rightarrow I I^{*}(N, L),
$$

çue ó cliamada aplicação inudice.

Assim tomos o soguinde par $\left(H^{*}(N, L), f_{N L}^{*}\right)$ á 1 mo oljeto on $\mathcal{E}$ c cstamos on condiçõos de aplicar a Redução do Lerny neste par.

O próximo resultado é nun pouto importantce da tcoria, pois inostra que a Redução (le Leray aplicada ao par $\left(H^{*}(N, L), f_{N L}^{*}\right)$ produ\% un objeto em $\mathcal{E}$ que independe da escollar do par-indice. 
Teorema 1.40. Sejam $(N, L)$ e $\left(N^{\prime}, L^{\prime}\right)$ marcs-inulices para um conjunto invariante isolado $S$. Entĩo $\mathcal{L}\left(H^{*}(N, L), f_{N l}^{*}\right)$ e $\mathcal{L}\left(H^{*}\left(N^{\prime}, L^{\prime}\right), f_{N^{\prime} l^{\prime}}^{*}\right)$ são oljetos isomorfos em $\mathcal{E}$.

Observação 1.41. Não apprcsentaremos a prova do tcorcma anterior. Ela faz uso do Proposição 1.37 e os detalhics pordem ser cucontrados em Mrozck [6].

Definição 1.42. Sejam $S$ un conjunto inuyariante isolado com relação a aplicação continuna $f: X \rightarrow X$ e $(N, L)$ un por-indice para $S$. O par

$$
\operatorname{Con}^{*}(S):=\mathcal{L}\left(H^{*}(N, L), \int_{N L}^{*}\right)
$$

é o chamado índice de Conley collamológico discreta para $S$.

Observação 1.43. Portanto o íuclice de Conley cohomológico para $S$ consiste de unu móclulo graduaclo e um isomorfismu distinguiclo. Na litcratura, este módulo é represcitiado por $C H^{*}(S, f)$ co isomortisuro por $\chi^{*}(S, f)$. Assim,

$$
\operatorname{Cou}^{*}(S)=\left(C H^{*}(S, f), \lambda^{*}(S, f)\right) \text {. }
$$

\subsection{Propriedades do Índice}

Três propriedades caracterizan o ínclice de Conley' $\mathrm{c}$ as descrevemos a seguir. Ao invés de apresentar suas demonstraçôses irenos ilustrar una aplicação das mesmas. Isso será fcito no Excmplo 1.47. Em Mrozek [6] encontrann-sc os detallics das provas dessins propriedades.

Proposição 1.44. (Propriedade de: Warcivski) $S e \operatorname{Con}^{*}(S) \neq \mathbb{O}$, então $S \neq \emptyset$.

Demonstração. Para a clemonstração basta nisar a Proposição 1.35 .

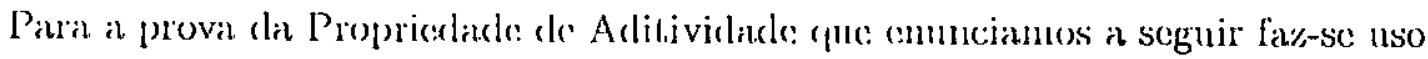
da Proposição 1.34.

Proposiçāo 1.45. (Propricelarle de Aditividadc) Suponha que $S_{1} \cup S_{2}$ seja um conjunto invariante isolado, onde $S_{1}$ e $S_{2}$ são conjuntas invariantes isolados disjunlos. Entäo $C H^{*}(S)$ é isonorfo a $C H^{*}\left(S_{1}\right) \times C H^{*}\left(S_{2}\right)$ e $\chi^{*}(S)$ é conjugada a. $\chi^{*}\left(S_{1}\right) \times \chi^{*}\left(S_{2}\right)$. 
En geral, cscrevemos ns conclusones da Proprieclade de Aclitiviclade de modo nuais resumido. A saber, $\operatorname{Con}^{*}(S)=\operatorname{Con}^{*}\left(S_{1}\right) \times \operatorname{Con}^{*}\left(S_{2}\right)$. Finalmonte, a terceira propriedacle clo índice:

Proposição 1.46. (Propricclacle (la Homotopia) Sejam $\Lambda \subset \mathbb{R}$ um inlervalo compaclo of $: \Lambda \times X \rightarrow X$ umn aplicação conlánua lal que para cada $\lambda \in \Lambda$

$$
\begin{aligned}
& f_{\lambda}: X \longrightarrow X \\
& x \longmapsto f(\lambda, x),
\end{aligned}
$$

scja uma aplicaçä́o conthinun e $N$ seja vizinhança isolante com relação o. $f_{\lambda}$, para

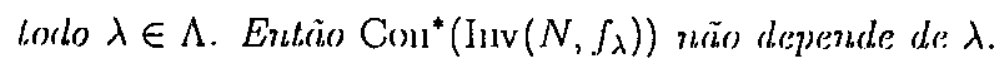

Encerramos a scçño conı nuna applicação das Propricdades cle Aditividade e Homotopia. Os detallıcs destc cxcmuplo são adaptações de um exemplo apresentado por Conley en [2].

Exemplo 1.47. Scja $f_{s}: \mathbb{R}^{2} \rightarrow \mathbb{R}^{2}$ uma faunília de aplicações contínuas dada por

$$
f_{s}\left(x_{1}, y_{1}\right)=\left(x_{1}, y_{1}\right)+s\left(y_{1}, x_{1}^{2}-1\right)
$$

oncle com $s \in[0, \varepsilon]$, coin $0<\varepsilon<1 / 2$. Note que se $s=0, f_{0}$ é a aplicação identidade. So $s \in(0, \varepsilon], f_{s}$ possui dois pontos fixos: $(-1,0)$ c $(1,0)$. Queremos saber se para $s \in(0, \varepsilon]$ lái alguma outua órbita invariaute para $f_{s}$. Para isso, faremos a seguinte mulança de variável

$$
x=s^{2} x_{1} \quad \circ \quad y=s^{3} y_{1}
$$

A nova cxpressão de $f_{s}$ tem a formal

$$
f_{s}(x, y)=(x, y)+\left(y, x^{2}-s^{4}\right)
$$

Alćm clisso, ć fácil ver cuuc as novas coordenadas dos pontos fixos de $f_{s}$ são

$$
\left(-s^{2}, 0\right) \circ\left(s^{2}, 0\right)
$$

Observe que fazcuclo $s=0$ na expressão en (1.4) não teremos a aplicaçāo identidade. Utilizando $f_{s}$ nas coordenadas $x$ c $y$, nosso olj.jctivo é mostrar que para todo $s \in[0, \varepsilon]$

$$
\operatorname{Inv}\left(N, f_{s}\right) \neq\left\{\left(-s^{2}, 0\right),\left(s^{2}, 0\right)\right\}
$$


onde $N=[-1 / 2,1 / 2] \times[-1 / 2,1 / 2]$. Primciranente, mostrenos que para $s \in[0, \varepsilon]$, $N$ ć unna viøinhança isolante para $f_{s}$. É fácil ver que para todo $x \in[-1 / 2,1 / 2] \mathrm{e}$ $y \in[-1 / 2,1 / 2]$, temos que $f(x, 1 / 2) \notin N$ e $f(1 / 2, y) \notin N$. Un cálculo um tanto tedioso, porém simples, permite conchluir que para cacla $(x, y)$ pertcucente à fronteira de $N$, cxiste $n_{0} \in \mathbb{N}$ tal que $f^{n_{0}}(x, y) \notin N$. Logo, se $s \in[0, \varepsilon]$, então $N$ é vizinlança isolante para $\operatorname{Inv}\left(N, f_{s}\right)$.

Parar calcular $\operatorname{Con}^{*}\left(\operatorname{luv}\left(N, f_{s}\right)\right)$ consturuintos muna nova faunília de aplicações cont.ímnas. Seja $y_{s}: \mathbb{R}^{2} \rightarrow \mathbb{R}^{2}$ (laulat por

$$
y_{s}(x, y)=(x, y)+\left(y, x^{2}+s^{4}\right)
$$

onde $s \in[0, \varepsilon]$. Através do un raciocínio reconrsivo verifica-se que $g_{s}^{n}=\left(\varphi^{n}, \psi^{n}\right)$ é unna aplicadgão cuja a segnnda coordenada ap)rescentar como forma geral

$$
\psi^{n}(u)=\sum_{j=1}^{n-1}\left(\varphi^{j}(u)\right)^{2}+\psi(u)+(n-1) s^{4} .
$$

Desta mancira, fica fácil ver que para todo $(x, y) \neq(0,0)$ c para todo $s \in(0, \varepsilon]$, temos que $\left\|y_{s}^{n}(x, y)\right\| \rightarrow \infty$, quando $u \rightarrow \infty$. O messno ć váliclo para $s=0$. Portanto, o conjunto compacto $N$ definido acima ó vizinhança isolante para $\operatorname{Inv}\left(N, g_{s}\right)$ e para todo $s \in[0, \varepsilon], \operatorname{Con}{ }^{*}\left(\operatorname{Inv}\left(N, g_{s}\right)\right)$ ć trivial. Como $f_{0}=g_{0}, \operatorname{temos}$ que $\operatorname{Con}\left(\operatorname{Inv}\left(N, f_{0}\right)\right)$ timulém é trivial. Definindo a aplicação contínua $F: \mathbb{R}^{2} \times[0, \varepsilon] \rightarrow \mathbb{R}^{2}$ dada por $F(s, u)=f_{s}(u)$, estaunos nas conclições da Propriedade de Honnotopia. Logo, para todo $s \in(0, \varepsilon], \operatorname{Con}\left(\operatorname{Inv}\left(N, f_{s}\right)\right)$ ć trivial.

Para finalizar, suponla que $\operatorname{Inv}\left(N, f_{s}\right)=\left\{\left(-s^{2}, 0\right),\left(s^{2}, 0\right)\right\}$. Pela Propriedade de Aditividade, temos que

$$
\operatorname{Con}\left(\operatorname{luv}\left(N, \int_{s}\right)\right)=\operatorname{Con}\left(\left\{\left(-s^{2},(0)\right\}\right) \times \operatorname{Con}\left(\left\{\left(s^{2}, 0\right)\right\}\right)\right.
$$

Porén é líkcil ver (que $\left(-s^{2}, 0\right)$ e $\left(s^{2}, 0\right)$ são pontos fixos hiperbólicos de $f_{s}$ (Definição 1.50). Logo, pelo Teorema 1.59, o laclo dircito de igualdade em (1.5) não ć trivial. Portanto, para carla $s \in(0, \varepsilon]$, cxiste no conjunto $N$ uma órbita limitada de $f_{s}$ distintia dos pontos fixos. 


\subsection{Ponto Fixo e Ponto Periódico Hiperbólico}

Nesta seção faremos o cálculo do índice de Conley para ponto fixo hiperbólico e ponto periódico hiperbólico de un difcomorfismo de classe $C^{1}$ definido $\mathrm{cm} \mathbb{R}^{n}$.

O cxemplo que seguc tein o intuito de preparar o lcitor para a demonstıaçãa do Tcorcuna 1.59, pois é um caso particular clo tcorema.

Exemplo 1.48. Seja $f$ a aplicação lincear definicla no Exemplo 1.3. Iremos calcular o índice de Conley do conjunto invariante isolado $S=\{(0,0)\}$. Para isto utilizarcmos o par-índice $(N, L)$ definiclo no Excmplo 1.11. Note que a matriz de $f$ na basc canônica de $\mathbb{R}^{2}$ é dada por

$$
\left(\begin{array}{cc}
2 & 0 \\
0 & 1 / 2
\end{array}\right)
$$

Denotarcinos por $E^{u}$ o subespaço de $\mathbb{R}^{2}$ gerado por $(1,0)$ c $E^{s}$ o subespaço de $\mathbb{R}^{2}$ grrado por $(0,1)$. Note cue $E^{s}$ c $E^{u}$ sĩo isomorfos a $\mathbb{R}$. Temos a decomposição $\mathbb{R}^{2}=E^{s} \oplus E^{u}$. Assin, $\left.f\right|_{E^{n}}$ tecon un autovalor com módulo maior do que 1. Já $\left.f\right|_{E^{s}}$ tem un alltovalor con módulo nenor do que 1. Por csta razão dizemos que $E^{u}$ é

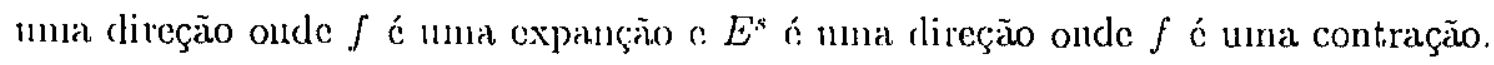
Salbelinos dute

$$
H^{q}(N, L)= \begin{cases}\mathbb{Q}, & \text { se } q=1 \\ 0, & \text { caso contrário. }\end{cases}
$$

Restat calcular a aplicação índicc. Note que precisanos nos preocupar somente com o caso $q=1$. Como $H^{1}(N, L) \cong \mathbb{Q}$, represcenticuremos por $\varphi$ o gerador de $H^{1}(N, L)$, onde $\varphi: H_{1}(N, L) \rightarrow \mathbb{Q}$ ć unıa aplicação linear. Sendo $H_{1}(N, L) \cong \mathbb{Q}$, tomamos $u$ como sen gerador. A teoria de cohomologia nos mostra que $f_{N L}^{*}(\varphi(u))=\varphi(u)$. Ou seja, $f_{N L}^{*}=$ Id. Logo,

$$
\operatorname{Col1}^{\prime}(\{(0,0)\})= \begin{cases}(\mathbb{Q}, 1 \mathrm{ld}), & \text { se } q=1 \\ 0, & \text { caso contrário }\end{cases}
$$

E o cxemplo estrí conchuido.

Iniciamos apresentando as definiçōes relativas no conceito de ponto fixo hiperbólico.

Definiçāo 1.49. Uma aplicaçŭo linear $A: \mathbb{R}^{n} \rightarrow \mathbb{R}^{n}$ é chamada de hiperbólica se, A é un isomorfismo e nenhlun anto-yalor de A tem nódulo igual a um. 
Definição 1.50. Se.ju $f: \mathbb{R}^{n} \rightarrow \mathbb{R}^{n}$ um difcomnorfismo de classse $C^{1}$. Um ponto $x \in \mathbb{R}^{n}$ é um ponto tixo hiporbólico se, $f(x)=x c c D f(x)$ é umu aplicaçăo linear hiperbólica.

Observação 1.51. O ponto 0 ć um ponto fixo hiperlbólico para uma aplicação lincar lıiperló́lica de $\mathbb{R}^{n}$ enı $\mathbb{R}^{n}$.

Definição 1.52. Scjum $f: \mathbb{R}^{n} \rightarrow \mathbb{R}^{n}$ um difeomorfismo de classe $C^{\prime}$ e $x$ u.m ponto furo hiperbólico de $f$. Denotaremos por $k$ o numero de auto-valores de $D f(x)$ com módulo menor que 1 , e denotaremos por l o número de auto-valores reais de $D f(x)$ menoress que -1. O par $(k, l)$ é chamado índice de Morse do ponto $x$.

Os Leınas $1.53,1.54$ c 1.56 scrão utilizados para o cálculo do Índice de Conley para um ponto fixo lipuperbólico de aplicações lineares hiperbólicas. Suas demonstraçōes podem ser encontradas en Palis c Melo [8].

Lema 1.53. Seja $A: \mathbb{R}^{n} \rightarrow \mathbb{R}^{n}$ umu aplicação linear hiperbólica. Então existe uma. decomposiçüu $\mathbb{R}^{n}=E^{s} \oplus E^{u}$ tal que $E^{s}$ e $E^{u}$ süo subespoços invariantes por $A$ e os autovalores de $A^{s}=\left.A\right|_{e^{s}}$ e $A^{\prime \prime}=\left.A\right|_{b^{\prime \prime}}$ são os autovolores de $A$ com módulo menor que um e moior que um, respectivarmenle.

Lema 1.54. Seja $A$ uma aplicação linecar hiperbólica. Então existe uma norma $\|\cdot\|$ em $\mathbb{R}^{n}$ tal que $\left\|A^{s}\right\|<1$ c $\left\|\left(A^{n}\right)^{-1}\right\|<1$.

Observação 1.55. O Lcolla 1.54 imlplica que $A^{\text {s }}$ ć uma contração c $A^{n}$ ć uma expialssio.

Lema 1.56. Se A é uına aplicaçüo linear hiperbólica $e \mathbb{R}^{n}=E^{s} \oplus E^{u}$ é a decomposicãa definida no Lema 1.53 , entăa $\left\{A^{n} x\right\}_{n=0}^{\infty}$ converge para a origem se $x \in E^{s}$ e $n \rightarrow \infty$ e $\left\{A^{-n} x\right\}_{n=0}^{\infty}$ converge para a origem se $x \in E^{u}$ e $n \rightarrow \infty$.

No próximo lenla, sgnt $(u)$ representa

$$
\operatorname{sgn}(u)= \begin{cases}1, & \text { se } u>0 \\ -1, & \text { se } u<0 .\end{cases}
$$

Lema 1.57. Seja $h: \mathbb{R} \times[0,1] \rightarrow \mathbb{R}$ a funuçăo definuida por

$$
h(u, l)=t u+(1-l) \operatorname{sgn}(u) \exp (\operatorname{sgn} n(|u|-1) \ln 2) .
$$

A funçăo h possui as seguinutes propriedlades: 
1. se $|u|>1$, ent.ão $|h(n, t)|>1$, para tedo $t \in[0,1] c$

2. se $|u|<1$, então $|h(u, t)|<1$, yara todo $t \in[0,1]$.

Demonstração. Para demonstrarmos 1 , scja $u \in \mathbb{R}$ tal que $u>1$. Logo, $\operatorname{sgn}(u)=$ 1 c sgn $(|u|-1)=1$. Assim a expressão para $h(u, t)$ se torna

$$
h(u, t)=t u+2(1-t)
$$

Consideremos o caso $u>2$. Então

$$
h(u, t)=(u,-2) t+2>2, \text { para todo } t \in[0,1] .
$$

Se $1<u<2$, entiño

$$
h(u, t)=(u-2) t+2>n>1, \text { para toclo } t \in[0,1]
$$

Agora suponha que $u$ ć $u$ múnero real tal quc $u<-1$. Faremos o mesmo tipo de análise para cste caso. Note que $\operatorname{sgn}(u)=-1 \operatorname{csg} n(|u|-1)=1$. Logo, a expressão para $h(u, t)$ se torma.

$$
h(u, t)=t u-2(1-t)
$$

É fácil vor cluc

$$
h(u, t)=t u+2 t-2<-t+2 t-2=t-2 .
$$

Logo,

$$
h(u, t)<-1, \text { parim torlo } 0<t<1 \text {. }
$$

Para (2), utiliza-sc ını raciocínio alıálogo.

Apresentamos o cálculo do Índice de Conley para o ponto fixo cle unna aplicação lincar hiperbólica. A seguir $\mathbb{G}$ denotará 1 mu grupo abeliano.

Proposição 1.58. Seja $A: \mathbb{R}^{n} \rightarrow \mathbb{R}^{n}$ uma aplicaçấo linear hiperbólica. Então $\{0\}$ é urn conjunto invariante isolado $e$

$$
\operatorname{Con}^{q}(\{0\})= \begin{cases}\left(\mathbb{G},(-1)^{l} \mathrm{Id}\right), & \text { se } q \neq k \\ 0, & \text { se } q=k\end{cases}
$$

onde $(k, l)$ é o índice de Morse de 0. 
DemonstraçÃo. Segue do Lemia l.53 (jue $\mathbb{R}^{n}$ pooke ser decomposto on (lois sul)espaços invariantes $E^{u}$ c $E^{s}$. Note çue $\operatorname{dim} E^{u}=k$ c dinı $E^{s}=n-k$. Logo, $E^{u}$ é isomorlo a $\mathbb{R}^{k}$ e $E^{s}$ é isomorlo a $\mathbb{R}^{n-k}$. Para sinuplificar à notação da demonstração utilizarcmos $\mathbb{R}^{k}$ e $\mathbb{R}^{n-k}$ para representar $E^{u}$ e $E^{s}$, respectivamente.

Pelo Lenla 1.56, temos que $S=\{0\}$ é o único conjunto invariante para $A$. Além disso, $S$ é um conjunto iıvariante isolado.

Vannos provar o caso chi que $A$ scja diagonalizável. Assim tomamos uma base de $\mathbb{R}^{n}$ tal que a matriz de $A$ nesta base tenha a forma bloco diagonal, com blocos unidimensionais $\left[\lambda_{i}\right]$ correspondentes aos autovalores rcais, $\mathrm{c}$ blocos bidimensionais compondentes ans antovalores complexos, os quais tomanuos ua forma.

$$
\left[\begin{array}{cc}
r_{j} \cos \varphi_{j} & r_{j} \operatorname{sen} \varphi_{j} \\
-r_{j} \operatorname{sen} \varphi_{j} & r_{j} \cos \varphi_{j}
\end{array}\right]
$$

correspodente ao par de autovalores $r_{j} \operatorname{cxp}\left(i \varphi_{j}\right) \mathrm{e} r_{j} \exp \left(-i \varphi_{j}\right)$. Para cada $t \in[0,1]$, clefina a aplicação $A_{l}$, de mancira que $A_{t}$ tculia a mesma forma bloco diagonal de $A$, cujo os blocos correspondentes aos autovalores reais têm a forma

$$
\left[h\left(\lambda_{j}, t\right)\right]
$$

c os blocos bidimensionais correspondentes aos autovalores complexos têm a forma

$$
h\left(r_{j}, t\right)\left[\begin{array}{cc}
\cos t \varphi_{j} & \operatorname{sen} t \varphi_{j} \\
-\operatorname{sen} t \varphi_{j} & \cos t \varphi_{j}
\end{array}\right]
$$

ondc $h(u, t)$ é a função definida no Lema 1.57 , ou scja ${ }_{7}$

$$
h(u, t)=t u+(1-t) \cdot \operatorname{sgn}(u) \exp (\operatorname{sgn}(|u|-1) \ln 2) .
$$

Pelo Lema 1.57 conchumos que para cadla $0 \leq t \leq 1,\{0\}$ é um conjunto invariante iscolaclo. Mais aindla, ó um ponton fixo hiperbólica coun índice cle Morse $(k, l)$. Note que $A_{1}=A$. Se $t=0$, un cálculo simples mostra que a matriz da aplicação $A_{0}$ assume a forma bloco diagonal, com os valores $2,-2,1 / 2,-1 / 2$ na sua diagonal. Em parlicular, $A_{0}$ restritan a $\mathbb{R}^{k}$ possui a forma

$$
\left[\begin{array}{rrrrrrrr}
2 & 0 & \cdot & \cdot & & \cdot & \cdot & 0 \\
0 & 2 & & & & & & \\
\cdot & & \cdot & & & & & \\
\cdot & & 2 & & & & \\
\cdot & & & -2 & & & \\
\cdot & & & & \cdot & & \\
\cdot & & & & & -2 & 0 \\
\cdot & \cdot & \cdot & . & . & \cdot & 0 & -2
\end{array}\right],
$$


conn $k-l$ inteiros positivos e $l$ intciros negativos. Sejan

$$
\begin{gathered}
N:=\left\{(x, y) \in \mathbb{R}^{k} \times \mathbb{R}^{n-k} \mid\|x\| \leq 2 \mathrm{c}\|y\| \leq 2\right\} \mathrm{e} \\
L:=\{x \in N \mid 2 \leq\|x\| \leq 1\}
\end{gathered}
$$

A demonstraçĩo de çuc $(N, L)$ é um parríndice invariante $\{0\}$ segue os mesmos passos realizados 110 Excmplo 1.11. Resta calcular o índice de Conley para $S=\{0\}$. Para isto, sejarn

$$
B^{k}:=\left\{x \in \mathbb{R}^{k} \mid\|x\| \leq 1\right\} \text { e } S^{k-1}:=\left\{x \in \mathbb{R}^{k} \mid\|x\|=1\right\}
$$

c defina as aplicações

$$
d:\left(B^{k}, S^{k-1}\right) \rightarrow\left(B^{k}, S^{k-1}\right) \text { e } \alpha:\left(B^{k}, S^{k-1}\right) \rightarrow(N, L),
$$

cladas por

$$
\begin{aligned}
d\left(x_{1}, x_{1}, \ldots, x_{k}\right) & =\left(x_{1}, x_{2}, \ldots, x_{k-l},-x_{k-l+1}, \ldots,-x_{k}\right) \\
\alpha(x) & =(x, 0) .
\end{aligned}
$$

Note que a aplicação clacla pela composição $i_{N L} \circ \alpha \circ d$ é homotópica à aplicação dadia pela composição $\alpha \circ A_{N L}$, onde a homotopia ć dada por

$H\left(\left(x_{1}, \ldots, x_{k}\right), t\right)=\left(2(1-t) x_{1}, \ldots, 2(1-t) x_{k-l},-2(1-t) x_{k-(l+1)}, \ldots,-2(1-t) x_{k}\right)$.

Logo, o diagrana a seguir ć homotopicanınte comutativo.

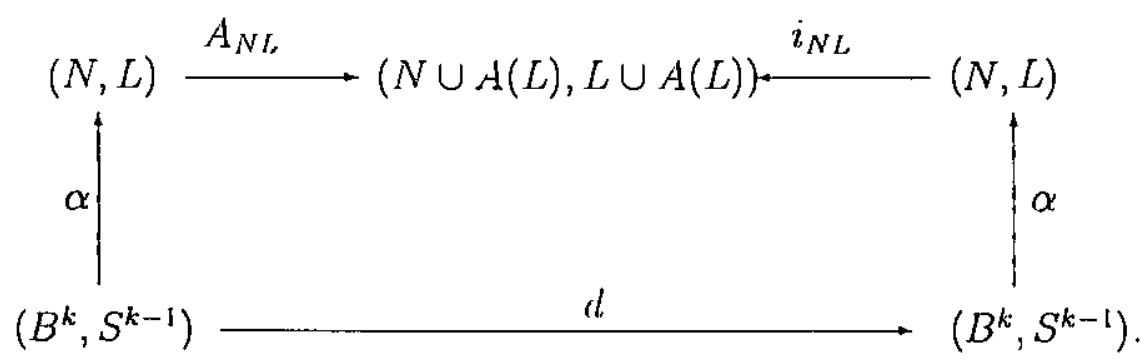

Consequentemente, o diaglama 


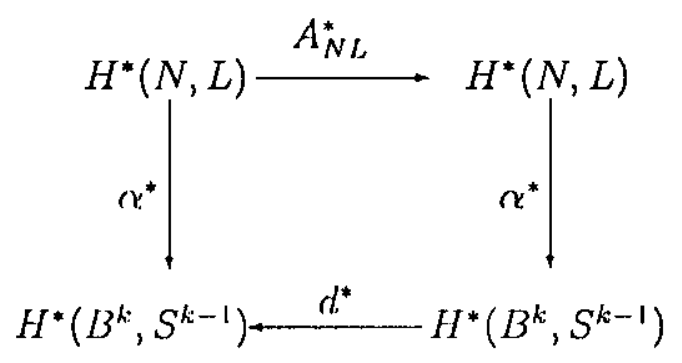

tambóm comutativo. Usando a proprieclade cla cxcisão forte da cohonologia de Alcxander-Spainer conclui-se que $\alpha^{*}$ ć $u m$ isomorfisıno. Assim

$$
\operatorname{Con}(\{0\})=\mathcal{L}\left(H_{N L}^{*}, A_{N L}^{*}\right)=\mathcal{L}\left(H\left(B^{k}, S^{k-1}\right), d^{*}\right)=\left(H^{*}\left(B^{k}, S^{k-1}\right), d^{*}\right),
$$

pois $d^{*}$ ć un isonorfismo. Ulilizando os Teorema 3.18 e $45.8 \mathrm{~cm}$ [7] temos que $\left(H^{*}\left(B^{k}, S^{k-1}\right), d^{*}\right)$ possui cxatamente os valores descjados. Agora basta aplicar a Propriclade de Homotopia c obtemos o resultado para o caso em que $A$ é diagonalizável.

Para o caso geral, tome cm $\mathbb{R}^{\prime \prime}$ uma base de modo que $A$ esteja na sua forma canônica de Jorclan real. Logo, csta matriz terá blocos da forma

$$
\left[\begin{array}{cccccc}
\lambda_{i} & & & & \\
1 & \lambda_{i} & & & \\
& & \cdot & & \\
& & & \cdot & \\
& & & \cdot & \\
& & & 1 & \lambda_{2}
\end{array}\right]
$$

correspondentes aos autovalores reais, e blocos da forma

$$
\left[\begin{array}{cccccc}
M_{i} & & & & \\
I & M_{i} & & & \\
& & \cdot & & \\
& & & \cdot & \\
& & & \cdot & \\
& & & I & M_{i}
\end{array}\right],
$$

onde

$$
M_{i}=\left[\begin{array}{ll}
r_{j} \cos \varphi_{j} & r_{j} \operatorname{sen} \varphi_{j} \\
-r_{j} \operatorname{sen} \varphi_{j} & r_{j} \cos \varphi_{j}
\end{array}\right] \text { e } I=\left[\begin{array}{ll}
1 & 0 \\
0 & 1
\end{array}\right]
$$

correpondentes aos autovalores complexos. Para cada $t \in[0,1]$, defina a aplicação linear $A_{l}$, de mancira que $A_{t}$ temlin a mesna forma hloco cliagonal de $A$, cujo os 
blocos correspondentes a.os autovalores reais têın a mesma ordem dos blocos reais de $A$, senclo que os núnueros $\lambda_{i}$ wĩuo trocaclos por $h\left(\lambda_{i}, t\right)$. Os blocos correspondentes aos antovalores complexos têu a mesima ordem dos blocos complexos de $A$, sendo (jue a matriz $M_{j}$ ć substituída por

$$
h\left(r_{j}, t\right)\left[\begin{array}{cc}
\cos t \varphi_{j} & \operatorname{sen} t \varphi_{j} \\
-\operatorname{sen} t \varphi_{j} & \cos t \varphi_{j}
\end{array}\right]
$$

c a nuatriz $I$, por $\left[\begin{array}{ll}t & 0 \\ 0 & t\end{array}\right]$.

Note que a matriz $A_{0}$ está nas condiçõcs do caso anterior. Como antcriormente, podemos aplicar a Propriedade de Homotopia c, portanto, obtemos o resultado descjado.

Finalmente apresentanıs o céllculo do Índice de Conley para um ponto fixo hiperbólico de um difeomorfismo de classe $C^{\mathbf{l}}$.

Teorema 1.59. Sejam $f: \mathbb{R}^{n} \rightarrow \mathbb{R}^{n}$ um difeomorfismo de classe $C^{1}$ e $x$ um ponto fixo hiperbólico de $f$. Enlío $S=\{x\}$ é un conjunto invariante isolado $e$

$$
\operatorname{Con}^{\prime \prime}(\{x\})= \begin{cases}\left(\mathbb{G},(-1)^{l} \mathrm{I} \mathrm{l}\right), & \text { se } q \neq k \\ 0, & \text { se } q=k\end{cases}
$$

onde $(k, l)$ é o indice de Marse de $x$.

Demonstração. Podemos supor sem perda de generalidade que $x=0$. Note que já sabemos calcular o Índice de Conley do ponto fixo hiperbólico de $A=D f(0)$. Assim, nosso objctivo é ntilizar a Propriedade clc Homotopia para concluir o resultado. Logo, necessitamos de um nucio de rclacionar $f$ com $D f(0)$. Essa relação é a essência do Toerema de Hartman-Grobınan (ver [8]).

Pelo Teorema de Hartinau-Grobnan, temos que, dado $\varepsilon>0$, existe $U_{\varepsilon}$, uma vizinlıança de 0 tal que para todo $x \in U_{\varepsilon}$,

$$
f(x)=A x+r(x), \operatorname{com}\|r\|<\varepsilon .
$$

Seja $\Lambda \in[0,1]$. Defina a família de aplicações

$$
f_{\lambda}(x):=A x+\lambda r(x)
$$


Note que $H(x, \lambda)=f_{\lambda}(x)$ definc una homotopia entre as aplicações $A$ e $f$. Pela Proposiçāo 1.58, sabcmos calcular $\operatorname{Con}^{*}(\{0\}, A)$. Para utilizar a Propriedade de Homotopia devemos encontrar um conjunto cornpacto $N \subset \mathbb{R}^{n}$ tal que $N$ é uma vizinhança isolantc para $f_{\lambda}$, para toclo $\lambda \in[0,1]$. Inicialmente, para cada $\lambda \in[0,1]$, vamos determinar una vizinhanģa isolanto $N_{\lambda}$.

Para cada $\lambda \in[0,1]$, o Terema rle Hartiman-Grobman implica que, dado $\varepsilon>0$ existc $\delta(\lambda)>0$ tal que

$$
f_{\lambda}(x)=A x+\lambda r(x) \text { com }\|v\|<\varepsilon \text { para todo } x \in B(0, \delta(\lambda))
$$

c ainda, cxiste um homeomorfismo $h_{\lambda}: B(0, \delta(\lambda)) \rightarrow B(0, \delta(\lambda))$ tal que

$$
A \circ h_{\lambda}=h_{\lambda} \circ f_{\lambda} \text {. }
$$

Tome $N_{\lambda}:=\operatorname{cl}(B(0, \delta(\lambda) / 2))$ c $0 \in N_{\lambda}$. Alćm disso, para cada $\lambda \in[0,1]$, temos (que $f_{\lambda}(0)=0$. On seja, 0 ś um ponto lixo para $f_{\lambda}$. Logo, $\{0\} \subset \operatorname{Inv}\left(N_{\lambda}, f_{\lambda}\right)$. Por ontro lado, scja $x \in \operatorname{Inv}\left(N_{\lambda}, f_{\lambda}\right)$. Logo, existe una secuência $\left\{x_{n}\right\}_{-\infty}^{\infty} \subset N_{\lambda}$ tal que $f_{\lambda}\left(x_{n}\right)=x_{n+1}$ e $x_{0}=x$. Assim, $f_{\lambda}^{n}(x) \in N_{\lambda}$, para todo $n \in \mathbb{Z}$.

Note também que $A\left(h_{\lambda}(0)\right)=h_{\lambda}\left(f_{\lambda}(0)\right)=h_{\lambda}(0)=0$. Mas como $A$ posssui um único ponto fixo, temos que $h_{\lambda}(0)=0$. Logo, $0 \in h_{\lambda}\left(N_{\lambda}\right)$. Sendo $h_{\lambda}$ um homeomorfismo temos que $h_{\lambda}\left(N_{\lambda}\right)$ ć um conjunto compacto. Un fato de fácil verificação ć que

$$
A^{n} \circ h_{\lambda}=h_{\lambda} \circ \int_{\lambda}^{n} \text { para todo } n \in \mathbb{Z} \text {. }
$$

Como $f_{\lambda}^{n}(x) \in N_{\lambda}$, para todo $n \in \mathbb{Z}$, temos que

$$
A^{n}\left(h_{\lambda}(x)\right) \in h_{\lambda}\left(N_{\lambda}\right) \text { para todo } n \in \mathbb{Z}
$$

isto é,

$$
h_{\lambda}(x) \in \operatorname{Inv}\left(h_{\lambda}\left(N_{\lambda}\right), A\right)=\{0\} .
$$

Como $h_{\lambda}$ ó homeomorfismo, temos quie $x:=0$. Logo, $\operatorname{Iuv}\left(N_{\lambda}, f_{\lambda}\right)=\{0\}$. Portanto,

$$
\operatorname{Inv}\left(N_{\lambda}, f_{\lambda}\right) \subset \operatorname{Int}\left(N_{\lambda}\right) \text { para todo } \lambda \in[0,1] \text {. }
$$

Un argumento de compaciclacle nos permite encontrar $\delta>0$ tal que $N_{\delta}$ ć vizinhança isolante para $\operatorname{Inv}\left(N_{\delta}, f_{\lambda}\right)$ c $\operatorname{Inv}\left(N_{\delta}, f_{\lambda}\right)=\{0\}$, para todo $\lambda \in[0,1]$. Como $f_{1}=f$ e $f_{0}=A$ segue da a Propriedacle de Homotopia quc

$$
\operatorname{Con}\left(\operatorname{Inv}\left(N_{\delta}, f\right)\right)=\operatorname{Con}\left(\operatorname{Inv}\left(N_{\delta}, A\right)\right)
$$


E a demonstração está concluída.

Passeınos a definição de ponto periódico hiperbólico.

Definição 1.60. Seja $f: \mathbb{R}^{n} \rightarrow \mathbb{R}^{n}$ um difeomorfismo de classe $C^{1}$. Um ponto $x$ é um ponto perióclico hiperbólico de periodo minimal d se $x$ for um ponto fixo hiperbólico para $f^{d}$ e se $i<d$, entä̃o $f^{i}(x)=f^{j}(x)$, para algum $j=1,2, \ldots, d-1$.

Teorema 1.61. Sejam $f: \mathbb{R}^{n} \rightarrow \mathbb{R}^{n}$ um difeomorfismo de classe $C^{1}$ e $x \in \mathbb{R}^{n}$ um ponto periódico de periodo minimal d de $f$. Suponha que $(k, l)$ seja o índice de Marse de $x$, com relação a $f^{i}$. Entăo $S=\left\{x, f(x), f^{2}(x), \ldots, f^{(l-1}(x)\right\}$ é um conjunto invariante isolado $e$

$$
\operatorname{Con}^{q}(S)= \begin{cases}\left(\mathbb{G}^{d}, D\right) & \text { se } q=k \\ 0, & \text { se } q \neq k\end{cases}
$$

onde $D: \mathbb{G}^{d} \rightarrow \mathbb{G}^{d}$ está definidla cm uma base $B=\left\{e_{1}, e_{2}, e_{3}, \ldots, e_{d l}\right\}$ de $\mathbb{G}^{d}$, por $D\left(e_{i}\right)=e_{i+1}$, para $i=1,2, \ldots, d-1$ e $D\left(e_{d l}\right)=(-1)^{\prime} e_{1}$.

Demonstração. Coino $x$; ć um ponto fixo hiperbólico segundo $f^{d}$, existe $N$ uma vizinhança isolante para o conjunto $\{x\}$ scgundo $f^{t}$. Ou seja, existe $N \subset X$ compacto tal que $\{x\}=\operatorname{Inv}\left(N, f^{d}\right)$. Denotcuos por $(N, L)$ um par-índice para o conjunto invariante isolado $\{x\}$. Para cacla $i=1,2, \ldots, d-1$, defina

$$
x^{i}:=f^{i}(x), N^{i}:=f^{i}(N) \text { \& } L^{i}:=f^{i}(L) .
$$

O conjunto $N$ é escolliclo de tal nodo (que podernos supor que $N^{i} \cap N^{j}=\emptyset$, se $i \neq j$ e $f\left(N^{i}\right) \cap N^{j}=\emptyset$, se $j \neq(i+1) \bmod d$.

Note que $f^{d}\left(x^{i}\right)=f^{d}\left(f^{i}(x)\right)=f^{i}(x)=x^{i}$, e mais $D f^{d}\left(x^{i}\right)=D f^{d}(x)$. Ou seja, $x^{i}$ é ponto fixo lipiperbólico sogundo $f^{\prime}$, com índice de Morse $(k, l)$, para cada $i=0,1,2 \ldots, d-1$.

Como, para cada $i=1,2, \ldots, d-1, f^{i} \dot{c}$ um homeomorfismo, temos que $N^{i}$ e $L^{i}$ são conjuntos conıpactos. Vamos mostrar que $\left(N^{i}, L^{i}\right)$ é par-índice para $\left\{x^{i}\right\}$ com relaçĩo a $f^{\prime \prime}$.

Temos que $\operatorname{Inv}\left(N^{i}, f^{\prime}\right)=\left\{x^{i}\right\}$. De fato, se $y \in \operatorname{Inv}\left(N^{i}, f^{d}\right)$, existc sequência $\left\{y_{n}\right\}_{-\infty}^{\infty} \subset N^{i}$ tal que $f^{d}\left(y_{n}\right)=y_{n+1}$ e $y_{0}=y$. Logo, $y_{n} \in f^{i}(N)$, para todo $n \in \mathbb{Z}$. Assin, para todo $n \in \mathbb{Z}$, t.cinos que $y_{n}=f^{i}\left(z_{n}\right)$, para algunn $z_{n} \in N$. Portanto, 
$z_{n}=f^{-i}\left(y_{n}\right) \in N$, para todo $n \in \mathbb{Z}$. E nuais, $f^{d}\left(z_{n}\right)=f^{d}\left(f^{-i}\left(y_{n}\right)\right)=f^{-i}\left(f^{d}\left(y_{n}\right)\right)=$ $f^{-i}\left(y_{n+1}\right)=z_{n+1}$ c $z_{0}=f^{-i}(y)$. Desta mancira, tcmos que $f^{-i}(y) \in \operatorname{Inv}\left(N, f^{d}\right)$. Como $\operatorname{Inv}\left(N, f^{d}\right)=\{x\}$, entào $y=f^{i}(x)$. Logo, $y=x^{i}$. Agora, note que a scquência $\left\{x_{n}^{i}\right\}_{-\infty}^{\infty}$ tal quc $x_{n}^{i}=x^{i}$, parlı́ toclo $n \in \mathbb{Z}$, cstá inteiramentc contida ein $N^{i}, \operatorname{com} f^{d}\left(x_{n}^{i}\right)=x^{i}=x_{n+1}^{i}$ e $x_{0}^{i}=x^{i}$. Portanto, $x^{i} \in \operatorname{Inv}\left(N^{i}, f^{d}\right)$. Então, $\operatorname{Inv}\left(N^{i}, f^{d}\right)=\left\{x^{i}\right\}$.

Como $\operatorname{cl}\left(N^{i} \backslash L^{i}\right) \subset N^{i}$, temos que $\operatorname{Inv}\left(\operatorname{cl}\left(N^{i} \backslash L^{i}\right), f^{d}\right) \subset \operatorname{Inv}\left(N^{i}, f^{d}\right)=\left\{x^{i}\right\}$. Comı 110 parágrafo anterior, mostra-sc que $\left\{x^{i}\right\} \subset \operatorname{Inv}\left(\operatorname{cl}\left(N^{i} \backslash L^{i}\right), f^{d}\right)$. Portanto, $\operatorname{Inv}\left(\operatorname{cl}\left(N^{i} \backslash L^{i}\right), f^{d}\right)=\left\{x^{i}\right\}$.

Como $x \in \operatorname{Int}(N \backslash L)$, cxistee $r>0$ tial quc $B(x, r) \subset N \backslash L$. Sendo $f^{i}$ um homeomorfismo, tcmos que $f^{i}(B(x, r))=B\left(f^{i}(x), r^{i}\right)$, para algum $r^{i}>0$. Assim, $B\left(f^{i}(x), r^{i}\right) \subset f^{i}(N \backslash L) \subset f^{i}(N) \backslash f^{i}(L)$. Logo, $y=f^{i}(x) \in \operatorname{Int}\left(N^{i} \backslash L^{i}\right)$. Isto conclui a verificação do primeiro itenu da Definição 1.9 .

Agora, como $f^{d}(L) \cap N \subset L$ e fi ć mu homconorfismo, seguc que, $f^{i}\left(f^{d}(L)\right) \cap$ $N) \subset f^{i}(L)$. Ou seja, $f^{d}\left(f^{i}(L)\right) \cap f^{i}(N)=f^{d}\left(L^{i}\right) \cap N^{i} \subset L^{i}$. O quc conclui o itcm dois da definição elc par-índice.

Para conferimos o itcm restante, bastia notar que $N^{i} \backslash L^{i} \subset f^{i}(N \backslash L)$. Logo, $f^{d}\left(N^{i} \backslash L^{i}\right) \subset f^{d}\left(f^{i}(N \backslash L)\right) \subset L^{i}$.

Desta maneira, seguc claranuente cule

$$
M:=\bigcup_{0 \leq i \leq d-1} N^{2}
$$

é vizinhança isolante para $\left\{x^{0}, x^{1}, \ldots, x^{d-1}\right\}$ com relação a $f$. Além disso, definindo

$$
Q:=\bigcup_{0 \leq i \leq d-1} L^{i}
$$

teınos que, $(M, Q)$ ć uın par-índicc para $\operatorname{Iıv}(M, f)$. Pcla construção do par $(M, Q)$, segue que

$$
H^{q}(M, Q)=H^{q}\left(N^{0}, L^{0}\right) \times H^{q}\left(N^{1}, L^{1}\right) \times \ldots \times H^{q}\left(N^{d-1}, L^{d-1}\right) .
$$

Logo,

$$
H^{q}(M, Q)= \begin{cases}\mathbb{G}^{d}, & \text { se } q=k \\ 0, & \text { se } q \neq k\end{cases}
$$


Para finalizar a demonstração, resta detcrminar a aplicação indicc $f_{M Q}^{*}$. Para isso escolha $\alpha^{d-1}$ um gerador de $H^{k}\left(N^{d-1}, L^{d-1}\right)$. Por recorrĉncia defina a sequência cle geradores de $H^{k}\left(N^{i}, L^{2}\right)$ dada por $\alpha^{i-1}=f_{N L}^{*}\left(\alpha^{i}\right)$, para $i=0,1, \ldots, d-1$. Seja $u^{i}:=\left(0,0, \ldots, \alpha^{i}, \ldots, 0\right) \in H_{N^{i} L^{\prime}}^{*}$. Então a secutência $\left\{u^{i}\right\}_{0 \leq i \leq d-1}$ forma uma basc parn $H^{\prime \prime}(M, Q)$. Verifica-sc facilmente que

$$
\int_{M Q}^{*}\left(u^{i}\right)=u^{i+1}, \text { para } i=1,2, \ldots, d-1 .
$$

Para $u^{0}=\left(\alpha^{0}, 0, \ldots, 0\right)$, temos que

$$
f_{M Q}^{*}\left(u^{0}\right)=\left(f_{M Q}^{d}\right)^{*}\left(0,0, \ldots,\left(f_{N I}^{d l}\right)^{*}\left(\alpha^{d-1}\right)\right)=\left(0,0, \ldots,(-1)^{l} \alpha^{d-1}\right)=(-1)^{l} u^{d-1}
$$

Logo, $f_{M Q}^{*}$ ć $u m$ isomiorfismo. Para concluir a prova defina $D:=f_{M Q}^{*}$.

Finalizimuos o canjútulo com o cílculo do índice para a Ferradıra de Sinale o a $G$-Fcrradura. Utilizanos $\mathbb{Q}$, conjunto dos múmeros racionais, para o cálculo dos grupos de colıomologia.

Exemplo 1.62. Scjaın $X^{-}=S^{2}=\mathbb{R}^{2} \cup\{\infty\}$ e $f: X \rightarrow X$ uma aplicação contínua que aplica dois retángulos $R_{0}$ c $R_{1}$ linearmente sobre $Q_{0}$ e $Q_{1}$ como mostra a Figura 1.3 .
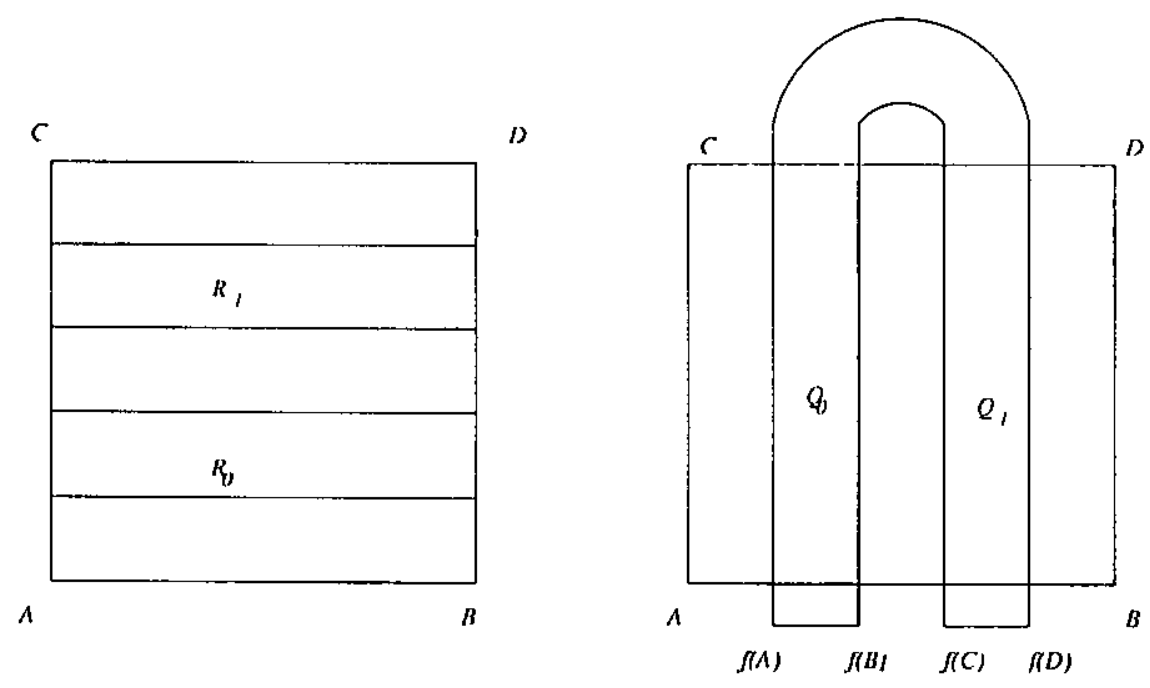

Figurn 1.3: A Fcrraclura cle Sinale.

Para maiores detallucs sobrc a construção da aplicação $f$ em $X$ ver Palis e Melo [8]. Esta aplicação ć chamada de Ferradıra de Snıale. Estamos intcressados em determinar o índice de Conley para $\operatorname{Inv}(N)$, onde $N$ ć o quadrado $A B C D$. A 
clescrição da dinâmica de $f$ em $N$ ć bcm conlıccida c pode ser cncontrada em [8]. Para o cálculo do índice cle Conlcy de $\operatorname{Inv}(N)$ precisamos de um par-índice. $\mathrm{Na}$ Figura 1.4 encontra-se uma represcntação geométrica cle um par-índice para $\operatorname{Inv}(N)$.

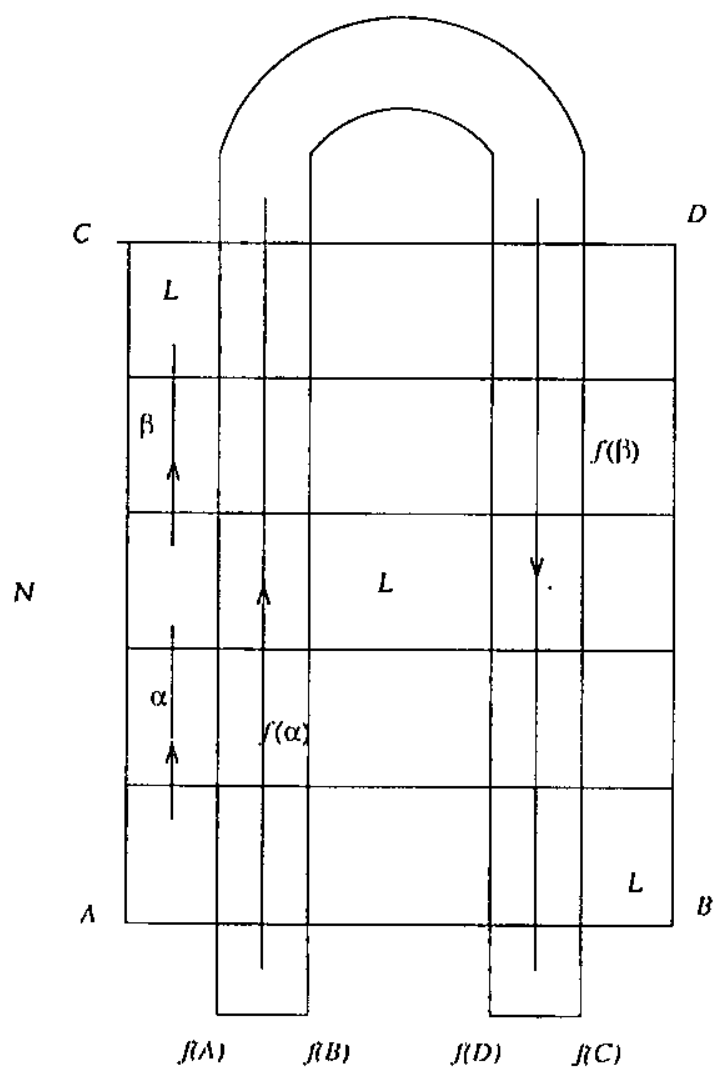

Figura 1.4: Unil par-indice para a Ferradura de Smale.

Para mostrau que $(N, L)$ ć cle fato un par-índicc para $\operatorname{Inv}(N, f)$, seja $x \in \operatorname{Inv}(N)$ c suponha que $x \in\left(\operatorname{cl}(N \backslash L)^{c}\right)$. Desta mancira são necessárias apenas duas iterações de $f$ para (que saia cle $N$, isto é, $f^{2}(x) \notin N$. O que ć uma contradição. Logo, $x \in \operatorname{cl}(N \backslash L)$. Repetindo os nucsmos argumentos para $x_{n}$ tal que $f\left(x_{n}\right)=x_{n+1}$, $x_{0}=x$ concluiunos o mesmo. Logo, a sequência $\left\{x_{n}\right\}$ está intciramente contida em $N$. Portanto, $x \in \operatorname{Inv}(\operatorname{cl}(N \backslash L))$. É claro que $\operatorname{Inv}(\operatorname{cl}(N \backslash L)) \subset \operatorname{Inv}(N)$. Resta mostrar que $\operatorname{Inv}(N) \subset \operatorname{Int}(N)$. Tonuc $x \in \operatorname{Inv}(N)$. Logo, existe $x_{-1} \in \operatorname{cl}(N \backslash L)$ tal que $f\left(x_{-1}\right)=x$. Portanto, $x \in N \cap f(N)$. Mas sc $x$ está ua fronteira de $N \cap f(N)$, cuttão $f(x) \notin N$, ou seja, $f^{2}\left(x_{-1}\right) \notin N$. As ontras propricdades de par-índice são de fácil vcrificação. 
Temos que

$$
l^{\prime \prime}(N, L)= \begin{cases}\mathbb{Q} \times \mathbb{Q}, & \text { so } q=1 \\ 0, & \text { (asso (ontrinios. }\end{cases}
$$

Novamente, para o crílculo do indice cle Conley, basta considerar $q=1$. Na Figura 1.4, a c $\beta$ denotam representintes dos gcradores de $H_{1}(N, L)$. Temos que a aplicação inchuzida pode ser apresentiala pela malrim

$$
\left(\begin{array}{ll}
1 & -1 \\
1 & -1
\end{array}\right)
$$

Um cálculo simples inostra que $\left(f_{N L}^{*}\left(H^{1}(N, L)\right)\right)^{2}$ ć a matriz nula. Portanto,

$$
\frac{H^{\prime}(N, L)}{\operatorname{gkcr}\left(f_{N L}^{*}\left(H^{1}(N, L)\right)\right)} \cong 0 .
$$

E assimı, a aplicação inıduzida no nível 1 tamıbém é nula. Ou seja, $\operatorname{Con}^{*}(\operatorname{Inv}(N))$ é trivial.

No próximo exemplo faremos ıma pequeıra modificação no exemplo anterior.

Exemplo 1.63. Seja $X^{r}$ como no Excmplo 1.62 c $f: X \rightarrow X$, uma aplicação contínua que aplica dois retângulos $R_{0}$ \& $R_{1}$ linearnente soble $Q_{0}$ c $Q_{1}$, como mostra a Figura 1.5. Esta aplicação é clamada $G$-Ferradura.
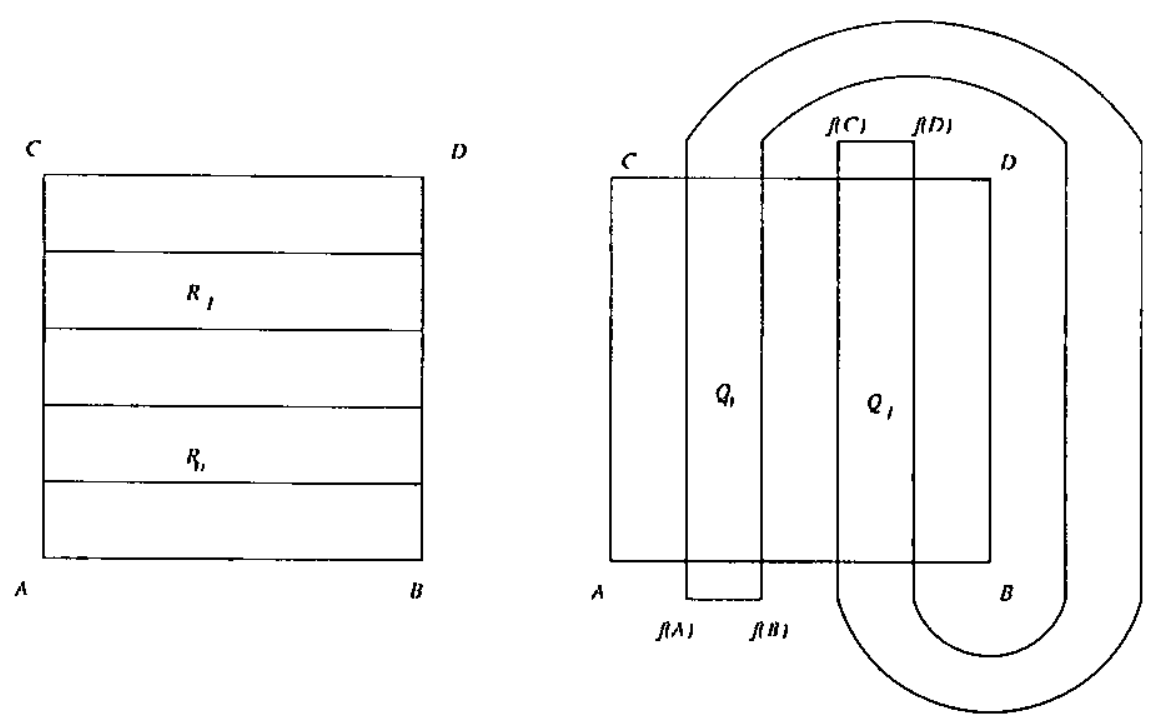

Figura 1.5: A G-Ferradura.

De maneira completauncntc alláloga io do Excmplo 1.62, mostra-sc que o par

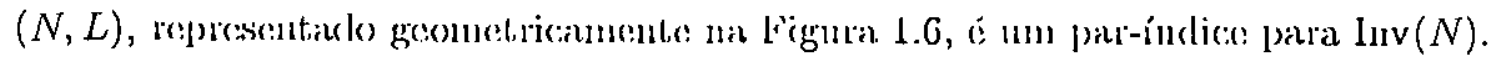




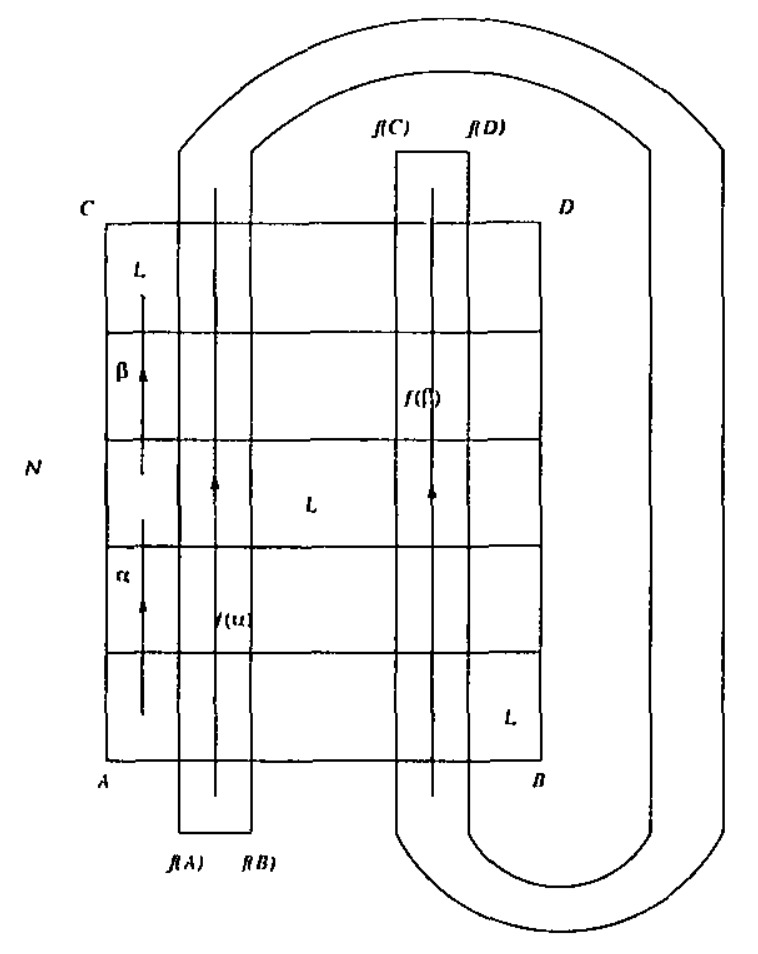

Figura 1.6: Um par-índicc para a $G$-Ferradura.

Como anteriormente,

$$
H^{\prime \prime}(N, L)= \begin{cases}\mathbb{Q} \times \mathbb{Q}, & \text { s: } q=1 \\ 0, & \text { caso contrário. }\end{cases}
$$

Tambćm aqui representarcmos a aplicação-índice na forma matricial. Para os niveis $q \neq 1$, temos que a aplicação índice ó nula. Já para o nível $q=1$, temos que a aplicaçāo índice é representada pola matriz

$$
\left(\begin{array}{ll}
1 & 1 \\
1 & 1
\end{array}\right)
$$

Na Figura 1.6 temos denotados $\alpha$ e $\beta$, representantes dos geradores de $H_{1}(N, L)$. Como estamos traballiando com cspaços vetoriais, os cálculos para o obtenção clo índice são cxtremamente simples. É fácil ver que $\left(f_{N L}^{1}\right)^{n}=2^{n-1} f_{N L}^{1}$. Logo, $\operatorname{gkcr}\left(f_{N L}^{1}\right)=\operatorname{ker}\left(f_{N L}^{1}\right)$. Portanto, $\operatorname{gker}\left(f_{N L}^{1}\right)$ ć gerado por $(1,-1)$. Tomando $\{(1,0),(-1,1)\}$ como uma basc para $H^{1}(N, L)$, temos que

$$
\frac{H^{1}(N, L)}{\operatorname{gker}\left(f_{N I}^{1}\right)} \cong[(1,0)] \text {. }
$$


Assim a aplicação $\left(f_{N L}^{1}\right)^{\prime}$ assume an forma $\left(f_{N L}^{1}\right)^{\prime}:[(1,0)] \rightarrow[(1,0)]$, dada por $\left(f_{N L}^{1}\right)^{\prime}((1,0))=\left[\left(f_{N L}^{1}\right)(1,0)\right]$. Mas $\left(f_{N L}^{\prime}\right)(1,0)=(1,1)=2(1,0)-1(-1,1)$. Logo, $\left[\left(f_{N L}^{\prime}\right)(1,0)\right]=2[(1,0)]$, isto $\dot{s},\left(f_{N L}^{\prime}\right)^{\prime}=2 \mathrm{Icl}$. Portiauto,

$$
\operatorname{Con}^{q}(\operatorname{Iuv}(N))= \begin{cases}(\mathbb{Q}, 2 \mathrm{I} \mathrm{l}), & \text { se } q=1, \\ 0, & \text { caso contrário. }\end{cases}
$$

Neste caso o índice não é trivial. 


\section{Capítulo 2}

\section{Decomposição Atratora-repulsora}

Neste capítulo apresentaremos a decomposição atratora-repulsora para um conjunto invariante isolado. Ela representa un caso particular da decomposição de Morse. Iniciamos introduzindo o conceito de conjuntos limites. O principal resultado deste capítulo está na Seção 3.2. A partir da existência de trio-índice para uma decomposiçäo atratora-repulsorá, ć construícla mua secpuencia exata longa (Teorema 2.13). Esta representa uma ferramenta para determinar órbitas de conexão entre os conjuntos limites (Corolário 2.15).

\subsection{Par Atrator-repulsor}

Nesta seção apresentaremos o conceito de conjunto limite e demonstraremos algumas propriedades desse conjunto. Finalizamos com o conceito de decomposição atratorarepulsora para un conjunto invariante isolado. As provas dos resultados abaixo constituem a versão discreta dos resultados provados por Rybakowski [12] para o caso de semifluxos contínuos definidos em espaços métricos.

Recordemos que $X$ denota um espaço métrico localmente compacto e $f: X \rightarrow X$ uma aplicação contínua.

Definição 2.1. Seja Y'um subconjunto de X'. O conjunto omega limite (w-limite) de $Y$ é definido como

$$
\omega\left(Y^{*}\right):=\bigcap_{n>0} \mathrm{cl}\left(\left\{f^{k}(x) \mid x \in Y^{*} \text { e } k \geq n\right\}\right) .
$$


A próxima proposiçào apresenta algumas propricdades básicas de conjunto omega limite.

Proposição 2.2. As seguintes afirmativass sào válidas:

1. Para todo $y \in X, y \in \omega\left(Y^{*}\right)$ se e somente se existem sequências $x_{k} \in Y e$ $n_{k} \in \mathbb{N}$ tais que $n_{k} \rightarrow \infty$ e $f^{n_{k}}\left(x_{k}\right) \rightarrow$ y quando $k \rightarrow \infty$.

2. O conjunto $\omega\left(Y^{*}\right)$ é fechado e $f\left(\omega\left(Y^{*}\right)\right) \subset \omega\left(Y^{*}\right)$.

3. Se Y'é um conjunto compacto e invartante, entào $\omega(Y) \neq \emptyset$ e $\omega\left(Y^{\circ}\right)$ é um conjunto invariante.

DemonstraÇÃo. Scja $y$ um elemento $\operatorname{cm} \omega\left(Y^{*}\right)$. Para cada $k \in \mathbb{N}$, temos que existe $y_{k} \in\left\{f^{j}(x) \mid x \in Y \quad\right.$ o $\left.j \geq k\right\}$ tal (que dist $\left(y_{k}, y\right)<\frac{1}{k}$. Portanto, existem $x_{k} \in Y$ e $n_{k} \geq k$ tais (que $y_{k}=f^{n_{k}}\left(x_{k}\right)$.

Por outro lado, suponha que $y \in X$ seja tal que existem sequências $x_{k} \in Y$ e $n_{k} \in \mathbb{N}$ tais que $n_{k} \rightarrow \infty$ e $f^{n_{k}}\left(x_{k}\right) \rightarrow y$ quando $k \rightarrow \infty$. Fixe $n \in \mathbb{N}$. Logo, existe $k_{0} \in \mathbb{N}$ (dependendo de $n$ ) tal que $n_{k} \geq n$ para $k \geq k_{0}$. Portanto,

$$
f^{n_{k}}\left(x_{k}\right) \in\left\{f^{j}(x) \mid x \in Y \text { e } j \geq n\right\},
$$

para todo $k \geq k_{0}$. Logo,

$$
y \in(l)\left(\left\{f^{j}(x) \mid x \in \mathcal{Y} \cdot j \geq n\right\}\right),
$$

para todo $n \in \mathbb{N}$. Com isso, a afirmativa 1 cestá provada.

Scja $y \in \mathrm{cl}\left(\omega\left(Y^{*}\right)\right)$. Darlo $\varepsilon>0$, existem $\bar{\eta} \in \omega\left(Y^{*}\right)$ c $k_{0} \in \mathbb{N}$ tais que para $k \geq k_{0}$ temos (1ue

$$
\operatorname{dist}(\bar{y}, y)<\frac{\varepsilon}{2} \quad \mathrm{c} \operatorname{dist}\left(f^{n_{k}}\left(x_{k}\right), \bar{y}\right)<\frac{\varepsilon}{2},
$$

onde $x_{k} \in U \cap S$ e $n_{k} \rightarrow \infty$. Portanto, $\operatorname{dist}\left(f^{n_{k}}\left(x_{k}\right), y\right)<\varepsilon$, para todo $k \geq k_{0}$. Logo, $y \in \omega\left(Y^{*}\right)$. A segunda parte da afirmativa 2 é innediata.

Sendo $Y$ invariante, temos que $f^{n}(y) \in Y$ para todo $y \in Y$ c $n \in \mathbb{N}$. A compacidade de $Y$ implica que toda sequência em $Y$ possui subsequencia convergente. Esses fatos, implicam que $\omega\left(Y^{*}\right)$ ć nào vazio. Para mostrar a invariância de $\omega(Y)$, 
bastia verificar cue $\omega\left(Y^{*}\right) \subset f\left(\omega\left(Y^{*}\right)\right)$. Scja $y \in \omega(Y)$. Portanto, existcm $x_{k} \in Y$ e $n_{k} \rightarrow \infty$ tiais que $f^{n_{k}}\left(x_{k}\right) \rightarrow y$. Como $n_{k} \rightarrow \infty$, cxistc $k_{0} \in \mathbb{N}$ tal que $n_{k}-1 \geq 0$ para todo $k \geq k_{0}$. Logo, $f^{n_{k-1}}\left(x_{k}\right) \in Y$ para todo $k \geq k_{0}$. Além disso, existe uma subscquência de $f^{n_{k-1}}\left(x_{k}\right)$ convergente. Denote por $x$ o limite dessa subsequência convergente. É fácil ver que $x \in \omega(Y)$ с $f(x)=y$.

No que segue $S$ denotará un conjunto compacto e invariante de $f$ e utilizaremos $\omega(x)$ para denotar o conjunto oncga limite de $\{x\}$.

Definição 2.3. Um conjunto $A \subset S$ é um atrator em $S$ se existe uma vizinhança $U$ de $A$ em $X$ tal que

$$
\omega(U \cap S)=A \text {. }
$$

$O$ conjunto $R:=\{x \in S \mid \omega(x) \cap A=\emptyset\}$ é chamado o repulsor dual de $A$ e o par $(A, R)$ é chamado deconıposição atratora-repulsora para o conjunto invariante $S$.

A proposição a scguir nos auxiliará na prova do principal tcorema desta seção.

Proposição 2.4. Seja $(A, R)$ um decomposição atratora-repulsora de S. Ent.ão as seguintes propriedades são válidas:

1. Sc $V$ é um conjunto aberto em $X$ com $A \subset V$, então existe um número natural $n_{0}=n_{0}(V)$ tal que $f^{n}(x) \in V$ para todo $x \in U \cap S$ e $n \geq n_{0}$.

2. Se $B$ é um conjunto fechado e disjunta de $A$, então para todo $\varepsilon>0$ existe um número natural $n_{0}=n_{0}(\varepsilon)$ tal que se $x \in S$ com $f^{n}(x) \in B$ para algum $n \geq n_{0}$, entĩo $\operatorname{dist}(x, R)<\varepsilon$.

DEMONSTRAÇÃo. Para a prova de 1 suponlıa que a afirmativa não seja verdadeira. Portanto, existe un aberto $V$ em $X$ com $A \subset V$ tal que para cada $k \in \mathbb{N}$, existem $x_{k} \in U \cap S$ e $n_{k} \geq k$ tais que $f^{n_{k}}\left(x_{k}\right) \notin V$.

Sem perda de generalidade, pockemos supor que $f^{n_{k}}\left(x_{k}\right) \rightarrow y$, quando $k \rightarrow \infty$, conl $y \in S \backslash V$. Portanto, $y \in \omega(U \cap S)=A$. Porém isso contradiz o fato que $y \notin V$.

Passemos à demonstração da afimativa 2. Novamentc, suponha que não seja verdadcira. Portanto, existem un conjunto fcchado $B$ c $\varepsilon_{0}>0 \mathrm{com}$ as seguintes propricdades: $B \cap A=\emptyset$ e para todo $k \in \mathbb{N}$, existe $x_{k} \in S$ com

$$
f^{n}\left(x_{k}\right) \in B \text {, para todo } n \geq k \text { c } \operatorname{dist}\left(x_{k}, R\right) \geq \varepsilon_{0} \text {. }
$$


Tomemos o conjunto aberto en $X$ \& $V=X \backslash B$. Portanto, $A \subset V$. Pela primeira partc desta proposição temos que cxiste un número natural $n_{0}:=n_{0}(V)$ tal que $f^{n}(x) \in V$ para todo $x \in U \cap S$ c $n \geq n_{0}$.

Sem perda de generalidade, podemos assumir que $x_{k} \rightarrow x$ c $x \in S$. A desigualdade em (2.1) inplica que

$$
\operatorname{dist}(x, R) \geq \varepsilon_{0}
$$

Portanto, $x \notin R$. Ou seja, $\omega(x) \cap A \neq \emptyset$. Logo, existe $j_{0} \in \mathbb{N}$ tal que $f^{j_{0}}(x) \in U \cap S$. Isso implica que existc $k_{0} \in \mathbb{N}$ tal que $f^{j_{0}}\left(x_{k}\right) \in U \cap S$ para todo $k \geq k_{0}$. Portanto,

$$
f^{n+j_{0}}\left(x_{k}\right) \in V \text { para torlo } n \geq n_{0} \text { c } k \geq k_{0} \text {. }
$$

Seja $\left\{n_{k}\right\}_{k=0}^{\infty}$ uma scquência tal que $n_{k} \rightarrow \infty$. Portianto, existe $\bar{k} \in \mathbb{N}, \bar{k} \geq k_{0}$ tal que para todo $k \geq \bar{k}$ tcmos que $n_{k} \geq n_{0}+j_{0}$ c $n_{k} \geq k$. Logo, sc $k \geq \bar{k}$, concluímos

$$
f^{n_{k}}\left(x_{k}\right)=f^{\left(n_{k}-j_{0}\right)+j_{0}}\left(x_{k}\right) \in V .
$$

Por outro lado, $f^{n_{k}}\left(x_{k}\right) \in B$. Porém isso ć uma contradição.

O teorcma a seguir possui uma demonstração simples e é baseada nas definições c resultados acima. Ele nos lá uma caracterização do comportamento dos elementos de $S$ em relação a uma decomposição atratora-1'spulsora.

Teorema 2.5. Seja $(A, R)$ um decomposição atratora-repulsora de S. Então

1. Os conjuntos $A$ e $R$ são disjunilos, compaclos e invariantes.

2. Se $y \in A$, enlão existe $y_{n} \in A$, com $n \in \mathbb{Z}$, lal quc $f\left(y_{n}\right)=y_{n+1}$ e $y_{0}=y$.

3. Sejam $y \in S$ e $y_{n} \in S$, conn $n \in \mathbb{Z}$, tais que $f\left(y_{n}\right)=y_{n+1}$ e $y_{0}=y$.

(a) Se $y \in R$, enlão $y_{n} \in R$ p para tado $n \in \mathbb{Z}$.

(b) Se $\omega(y) \cap R \neq \emptyset$, então $y_{n} \in R$ para todo $n \in \mathbb{Z}$.

(c) Se existe sequêthcia $\left\{n_{k}\right\}_{k=0}^{\infty}$ tal que $n_{k} \rightarrow \infty$ e $y_{-n_{k}} \rightarrow \bar{y} \in A$, então $y_{n} \in A$ para bodo $n \in \mathbb{Z}$.

(d) Se $y \notin A \cup R$, então $\omega(y) \subset A$ e para toda sequência $\left\{n_{k}\right\}_{k=0}^{\infty}$ tal que $n_{k} \rightarrow \infty e y_{-n_{k}} \rightarrow \bar{y}$ temos que $\bar{\eta} \in R$. 
Demonstração. Desde que $x \in A$ temos que $\omega(x) \subset A$. Portanto, $A \cap R=\emptyset$. A demostraçāo da invariâıcia de $A$ ć análoga à prova do item 3 da Proposição 2.2 . Como para todo $x \in X, \omega(x)=\omega(f(x))$, temos que $R$ ć também um conjunto invariantc. O itcm 2 da Proposiç̃̃o 2.2 implicar que $A \subset S$ é fechado. Sendo $S$ um conjunto compacto, temos que $A$ ć compacto.

Para encerrar a clemonstração da afimaliva 1, mostraremos que $R$ é um conjunto fecliado. Sejar $x_{k} \in R$ tal (ue $x_{k} \rightarrow x$ quaudo $k \rightarrow \infty$. Teuros que para todo $n \in \mathbb{N}$, $f^{n}(x) \notin U$. Caso contrário, existiriaan $n_{0}, k_{0} \in \mathbb{N}$ tais que $f^{n_{0}}\left(x_{k}\right) \in U \cup S$ para todo $k \geq k_{0}$. O que implicaria

$$
\omega\left(x_{k}\right)=\omega\left(f^{n_{0}}\left(x_{k}\right)\right) \subset \omega(U \cap S)=A,
$$

para todo $k \geq k_{0}$. Porćm isso contradiz a escolha da sequência $x_{k}$. Logo, para todo $n \in \mathbb{N}, f^{n}(x) \notin U$. Portanto, $x \in R$.

Suponha que $y \in A$. Se $n \geq 0$, defina $y_{n}:=f^{n}(y)$. Pela invariância de $A$, tcinos que $y_{n} \in A$, para todo $n \in \mathbb{N}$. Fixe $j \in \mathbb{N}$ e sejam $n_{k} \in \mathbb{N}$ e $y_{k} \in U \cap S$ sequências tais que $n_{k} \rightarrow \infty$ c $f^{n_{k}}\left(y_{k}\right) \rightarrow y$, quando $k \rightarrow \infty$. Logo, existe $k_{0}^{j} \in \mathbb{N}$ tal que $n_{k} \geq j$, para todo $k \geq k_{0}^{j}$. Como o conjunto $S$ é compacto, sem perda de gencralidade, podemos supor quc

$$
f^{n_{k}-j}\left(y_{k}\right) \rightarrow \bar{y}_{j} \in S
$$

quaudo $j \rightarrow \infty$. Logo, $\bar{y}_{j} \in A$ c $f^{j}\left(\bar{y}_{j}\right)=y$. Para cncerrar a demonstração, defina $y_{-j}:=\bar{y}_{j}$. Assim, concluínos a demoustração do segundo item do teorema.

Para a demonstração cla afirmativa 3 , scjain $y \in S$ e $y_{n} \in S$, com $n \in \mathbb{Z}$, tais que $f\left(y_{n}\right)=y, n$ с $y_{0}=y$.

Supoulia que $y \in R$. Scja $B$ uma vizinlıança fechacla de $R$ tal que $B \cap A=\emptyset$. Portanto, $f^{n}(x) \in B$, para todo $n \in \mathbb{N}$. Pela Proposição 2.4 temos que para todo $\varepsilon>0$ existe $n_{0} \in \mathbb{N}$ tal quc sc $x \in S$ e $f^{n}(x) \in B$ para algum $n \geq n_{0}$, então $\operatorname{dist}(x, R)<\varepsilon$. Fixemos $n \in \mathbb{Z}$. Portinto, cxiste $k \in \mathbb{N}$ tal que $k-n \geq n_{0}$. Com isso

$$
f^{k-n}\left(y_{n}\right)=f^{k}(y) \in B
$$

Logo, dist $\left(y_{n}, R\right)<\varepsilon$, para todo $\varepsilon>0$. Mas isso inplica que $y_{n} \in R$. Portanto, (a) estía provado. 
A demonstração de (b) é análoga a de (a). De fato, suponha $\omega(y) \cap R \neq \emptyset$. Portanto, existe $n_{k} \in \mathbb{N}$ sequêucial convergindo para infinito tal que $f^{n_{k}}(y) \rightarrow \bar{y} \mathrm{e}$ $\bar{y} \in R$.

Novamente, scja $B$ uma vizinlınugra fecliada cle $R$ tal que $B \cap A=\emptyset$. Portanto, existe $k_{0} \in N$ tal que $f^{n_{k}}(x) \in B$, para todo $n_{k} \geq k_{0}$. Pela Proposição 2.4 temos que para todo $\varepsilon>0$ existe $n_{0} \in \mathbb{N}$ tal que

$$
f^{\prime \prime}(x) \in B
$$

para todo $x \in S$ tal que dist $(x, R)<\varepsilon$ e $n \geq n_{0}$. Fixemos $n \in \mathbb{Z}$. Portanto, existe $n_{k}$ tal que $n_{k} \geq k_{0}$ c $n_{k}-n \geq n_{0}$. Com isso

$$
f^{n_{k}-n}\left(y_{n}\right)=f^{n_{k}}(y) \in B .
$$

Logo, $\operatorname{dist}\left(y_{n}, R\right)<\varepsilon$, para todo $\varepsilon>0$. Novanente, isso implica que $y_{n} \in R$.

Passemos à demonstraçãa de (c). Logo, suponhra que existe $n_{k} \in \mathbb{N}$ sequência convergindo para infinito tal que $y_{-n_{k}} \rightarrow \bar{y}$ e $\bar{y} \in A$. Portanto, existe $k_{0} \in \mathbb{N}$ tal que $y_{n_{k}} \in U \cap S$ para todo $n_{k} \geq k_{0}$.

Fixe $n \in \mathbb{Z}$. Qucremos mostrar cue $y_{n} \in A$. Seja $n_{k}$ tal que $n_{k}+n \geq 0$. Portanto, para todo $n_{k}$ temos que

$$
f^{n_{k}+n}\left(y_{-n_{k}}\right)=y_{n}
$$

Logo, $y_{n} \in \omega(U \cap S)=A$.

Finalmente, provemos (d). Suponlia $y \notin A \cup R$. Seja $x \in \omega(y)$. Logo, $n_{k} \in \mathbb{N}$ sequência convergindo para infinito tal que $y_{n_{k}} \rightarrow x$.

Afrrmamos que cxistc $n_{k_{0}} \in \mathbb{N}$ tal que $y_{n_{k_{0}}} \in U \cap S$. Caso contrário, $y_{-n} \in$ $S \backslash(S \cap U)$ para todo $n \in \mathbb{N}$. Tomando $B=S \backslash(S \cap U)$, a Proposição 2.4 implica quc para todo $\varepsilon>0$ cxiste un múmcro natural $n_{0}=n_{0}(\varepsilon)$ tal que dist $(x, R)<\varepsilon$ para todo $x \in S$ c $n \geq n_{0}$ tal que $f^{n}(x) \in B$. Logo

$$
\operatorname{dist}\left(f^{\prime \prime}\left(y_{n}\right), R\right)=\operatorname{dist}(y, R)<\varepsilon,
$$

para todo $\varepsilon>0$. Mas isso implica que $y \in R$, o que é uma contradição.

Como $\omega(U \cap S)=A$ c $A$ ć um conjunto invariante, temos que $y=f^{n_{k_{0}}}\left(y_{n_{k_{0}}}\right) \in A$. 
Para a segunda parte da afirnativa, scja $n_{k} \in \mathbb{N}$ sequência convergindo para infinito tal que $y_{-n_{k}} \rightarrow \bar{y}$. Qucremos mostrar (ne $\bar{y} \in R$. Considerc $B=\{y\}$. Pcla Proposição 2.4 tcmos que, para todo $\varepsilon>0$ existe um número natural $n_{0}=n_{0}(\varepsilon)$ tal que dist $(x, R)<\varepsilon$ para todo $x \in S$ o $n \geq n_{0}$ tal que $f^{n}(x) \in B$. Como $f^{n}\left(y_{n}\right)=y$, tcinos que $\operatorname{dist}\left(y_{n}, R\right)<\varepsilon$ para todo $n \geq n_{0}$. Logo, $\bar{y} \in R$.

O resultado anterior pode ser interpretado da seguinte forma. Se um conjunto compacto invariante possui ınıa decomposição atratora-repulsora, então uma das situações necessariamcute ocorte para cada ponto do conjunto invariante: ou ele está no atrator, ou cstá no repulsor, ou sua órbita nasce no repulsor e morre no atrator. Essa visão intuitiva motiva a scguinte definição:

Definição 2.6. Seja $(A, R)$ uma decomposição atratora-repulsora de um canjunto compacto e invariante $S$. O conjunto $S \backslash(A \cup R)$ é chamado o conjunto das órbitas de concxão de $S$. Esle conjunto será denolado yor $\mathrm{C}(A, R, S)$.

Uına conseçuência trivial da definição acima está rcgistrada a seguir.

Proposição 2.7. Seja $(A, R)$ umba decomposiçấo altralora-repulsora de um conjunto compacto e invariante $S$. Enlão $S=A \cup R \cup \mathrm{C}(A, R, S)$.

\subsection{Trio-índice para uma Decomposição Atrato- ra-repulsora}

Nesta seção vamos allalisar uma decomposição atratora-repulsora de um conjunto invariante isolado sob o ponto de vista da Teoria do Índice de Conley. Assim, o conjunto invariante scrá sempre isolado. O primciro resultado a ser demonstrado é a existência de trio-índice para uma decomposição atratora-repulsora. A partir da sequência exata longa construída na Proposição 2.13 concluímos o capítulo apresentando um resultado que ilustra como as informações sobre o índice podem garantir a cxistência de órbitas de conexão, isto é, o conjunto $\mathrm{C}(R, A ; S)$ ser não vazio. A cxposição desta seção é bascada cu Riclıeson [10].

Definição 2.8. Seja $(A, R)$ unna decomposição alratora-repulsora para o conjunto invariante isolado $S$. Um trio-íudicc para $(A, R)$ é um trio de conjuntos compactos 
$\left(N_{2}, N_{1}, N_{0}\right)$ tais que $\left(N_{2}, N_{0}\right),\left(N_{2}, N_{1}\right)$ e $\left(N_{1}, N_{0}\right)$ são pares-indices para $S, R$ e $A$, respectivamente.

Observação 2.9. Chamamos um trio-índice de trio-índice regular se os pares-índi$\operatorname{ccs}\left(N_{2}, N_{0}\right),\left(N_{2}, N_{1}\right)$ c $\left(N_{1}, N_{0}\right)$ são regulares.

O lcma apresentaclo a seguir nos anxiliará na prova da existência de trio-índice.

Lema 2.10. Seja $S$ um conjunlo invariante isolado e $(A, R)$ uma decomposição atratora-repulsora para $S$. Dado $(N, L)$ um par-indice regular para $S$, existem um conjunto compacto $U \subset N, \operatorname{com} A \subset \operatorname{Int}(U)$ e $\varepsilon_{0}>0$ tais que se $x \in$ $U$ e $\left\{x, f(x), f^{2}(x), \ldots, f^{\prime \prime}(x)\right\} \subset N$, enlãa $\operatorname{dist}\left(f^{i}(x), R\right)>\varepsilon_{0}$, para todo $i=$ $1,2, \ldots, n$.

DemonstraçĀo. Supoulia que para todo $U \subset N$ compacto, $\operatorname{com} A \subset \operatorname{Int}(U)$ e para todo $\varepsilon>0$, se $x$ é um clcmento de $U$ tal que $\left\{x, f(x), \ldots, f^{n}(x)\right\} \subset N$, então cxiste $i \in\{1,2, \ldots, n\}$ tal que $\operatorname{dist}\left(f^{i}(x), R\right) \leq \varepsilon$. Logo, cxiste uma sequência de conjuntos compactos $\left\{W_{k}\right\}_{k=0}^{\infty}$, com as seguintes propriedades:

$$
\begin{gathered}
W_{1} \subset U \cap S, \\
W_{k} \supset W_{k+1}, \\
A \subset \operatorname{Int}\left(W_{k}\right), \text { para toclo } k \in \mathbb{N} \mathrm{c}
\end{gathered}
$$$$
\text { sc } x \in W_{k} \text {, cutão dist. }(x, A)<\frac{1}{k} \text {. }
$$

Alénı disso, determinanıos $x_{k} \in W_{k}$ c $n_{k} \in \mathbb{N}$ tais quc

$$
f^{n_{k}}\left(x_{k}\right) \in N \text { c } \operatorname{dist}\left(f^{n_{k}}\left(x_{k_{k}}\right), R\right) \leq \frac{1}{k}
$$

Há dois casos a considerar. Primciro, suponla quc $\left\{n_{k}\right\}_{k=1}^{\infty}$ seja una sequência ilimitada, isto é, $n_{k} \rightarrow \infty$, quando $k \rightarrow \infty$. Notc quc $x_{k} \in U \cap S$, para todo $k \in \mathbb{N}$. Como $f^{n_{k}}\left(x_{k}\right) \in S$ e $S$ é unı conjunto compacto, existe uma subsequência $\left\{f^{n_{k_{j}}}\left(x_{k_{j}}\right)\right\}_{j=0}^{\infty}$ tal que $f^{n_{k_{j}}}\left(x_{k_{j}}\right) \rightarrow y$, quando $k_{j} \rightarrow \infty$. Além disso, $y \in S$. Mas temos que $\operatorname{dist}\left(f^{n_{k_{j}}}\left(x_{k_{j}}\right), R\right) \leq \frac{1}{k_{j}}$. O que implica que dist $(y, R)=0$. Como $R$ é um conjunto compacto, temos que $y \in R$. Por outro lado, $\left\{x_{k}\right\}_{k=1}^{\infty} \subset U \cap S$ é tal que dist $\left(f^{n_{k}}\left(x_{k}\right), y\right) \rightarrow 0$, quando $k \rightarrow \infty$. Logo, $y \in \omega(U \cap S)=A$. O que é uma coutradição. 
Resta considcrar o caso em que $\left\{n_{k}\right\}_{k=1}^{\infty}$ é uma sequência limitada. Portanto, cxistc $k_{0} \in \mathbb{N}$ tal que $n_{k}=n_{k_{0}}$, para todo $k \geq k_{0}$. Como, para todo $k \in \mathbb{N}, x_{k} \in S$ e $S$ é um conjunto compacto, então existe uma subsequência $\left\{x_{k_{j}}\right\}_{j=1}^{\infty}$ tal que $x_{k_{j}} \rightarrow x$, quando $k_{j} \rightarrow \infty$, e aincla mais $x \in S$. Recorde que a sequência $\left\{x_{k}\right\}_{k=1}^{\infty}$ foi cscolluida de tal forma que dist $\left(x_{k}, A\right)<\frac{1}{k}$. Concluinos que dist $(x, A)=0$. Portanto, $x \in A$. Por ontro lado, $\operatorname{dist}\left(f^{n_{0}}(x), R\right)=0$. Logo, $f^{n_{0}}(x) \in R$. O que nos leva, novamente, a uma contradição.

O resultado a seguir nos garante a cxistĉncia de trio-índice para um conjunto invariante isolado.

Proposição 2.11. Seja $S$ um, conjunto invariante isolado e $(A, R)$ sua decomposição atratora-repulsora. Então existc um trio-índice regulor para $(A, R)$.

Demonstração. Scudo $S$ unı conjunto invariante isolaclo, a Proposição 1.23 nos pcrmite concluir que, dada $V$, una vizinhança de $S$, cxistc $N \subset V$, bloco isolante tal que $\left(N, N^{-}\right)$é um par-indice regular para $S$. Defina

$$
N_{2}:=N \text { e } N_{0}:=N^{-}
$$

Dcvemos encontrar um conjunto compacto, $N_{1}$ tal que $\left(N_{2}, N_{1}, N_{0}\right)$ satisfaz as condições da Definição 2.8 .

Pelo lema anterior, cxisten $U \subset X$ compacto e $\varepsilon_{0}>0$, tais que $U \subset N_{2}$, $A \subset \operatorname{Int}(U)$ e se $x \in\left\{x, f(x), f^{2}(x), \ldots, f^{n}(x)\right\}$, então $\operatorname{dist}\left(f^{i}(x), R\right)>\varepsilon_{0}$, para todo $i=1,2, \ldots, n$. Considerc o conjunto

$$
Z:=\left\{y \in N_{2} \mid y=f^{n}(x) \text { para algum } x \in U \text { tal que }\left\{x, f(x), \ldots, f^{n}(x)\right\} \subset N_{2}\right\} \text {. }
$$

Defina

$$
N_{1}:=N_{0} \cup \operatorname{cl}(Z)
$$

Mostrenos que $\left(N_{2}, N_{1}, N_{0}\right)$ ć un trio-índice regular para $(A, R)$.

(i) O par $\left(N_{2}, N_{1}\right)$ é par-índice regular para $R$.

Seja $x \in R$. Logo, $x \in S$, ou scja, cxiste uma sequência $\left\{x_{n}\right\}_{-\infty}^{\infty} \subset \operatorname{cl}\left(N_{2} \backslash N_{0}\right)$ tal que $f\left(x_{n}\right)=x_{n+1}$ e $x_{0}=x$. Ein particular, $x \in \operatorname{cl}\left(N_{2} \backslash N_{0}\right)$, o que significa que cxistc unna sequência $\left\{y_{k}\right\}_{k=0}^{\infty} \subset N_{2} \backslash N_{0}$ tal que $y_{k} \rightarrow x$, quando $k \rightarrow \infty$. Portanto, 
para toclo $k \in \mathbb{N}$, tcmos que $y_{k} \notin N_{0}$. Suponla quc, para cada $k \in \mathbb{N}$, tenhamos

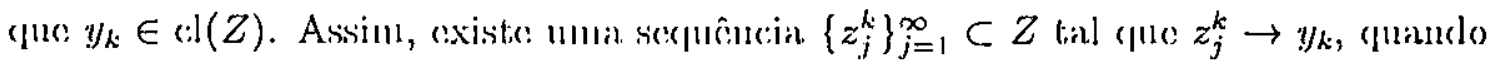
$j \rightarrow \infty$. Mas, para cadia $j, k \in \mathbb{N}$, tomos (lue $\operatorname{dist}\left(z_{j}^{k}, R\right) \geq \varepsilon_{0}$. Logo,

$$
\operatorname{dist}\left(y_{k}, R\right) \geq \varepsilon_{0}, \text { quando } j \rightarrow \infty
$$

Portinulio,

$$
\operatorname{clist}(x, R) \geq \varepsilon_{0}>0 \text {. }
$$

Isto gera uma contradição, pois $x \in R$. O que implica que $y_{k} \notin \mathrm{cl}(Z)$, para todo $k \in \mathbb{N}$. Portanto, $x \in \operatorname{cl}\left(N_{2} \backslash N_{1}\right)$. Utilizando o mesino argumento demonstramos qu1c

$$
x_{n} \in \operatorname{cl}\left(N_{2} \backslash N_{1}\right), \text { para todo } n \in \mathbb{Z} .
$$

Ou seja, $x \in \operatorname{Inv}\left(\operatorname{cl}\left(N_{2} \backslash N_{\mathrm{l}}\right)\right)$.

Resta mostrar que $\operatorname{Inv}\left(\operatorname{cl}\left(N_{2} \backslash N_{1}\right)\right) \subset R$ para concluirmos a verificação de parte do primeiro item da Definição 1.9. Para tanto, tome $x \in \operatorname{Inv}\left(\operatorname{cl}\left(N_{2} \backslash N_{1}\right)\right)$. Logo, cxiste una sequêucia $\left\{x_{n}\right\}_{-\infty}^{\infty} \subset \operatorname{cl}\left(N_{2} \backslash N_{1}\right)$ tal que $f\left(x_{n}\right)=x_{n+1}$ e $x_{0}=x$. Em particular, $x \in \operatorname{cl}\left(N_{2} \backslash N_{1}\right)$. Portanto, existe uma sequência $\left\{y_{i}\right\}_{i=1}^{\infty} \subset N_{2} \backslash N_{1}$ tal que $y_{i} \rightarrow x$, quando $i \rightarrow \infty$. On scja, $y_{i} \in N_{2}$ e $y_{i} \notin N_{1}$, para todo $i \in \mathbb{N}$. Como $(A, R)$ ć uma decomposição atratora-rcpulsora para $S$, há três possibilidades a serem consideradas:

$$
\begin{aligned}
& \text { (a) } x \in A, \\
& \text { (b) } x \in R \mathrm{e} \\
& \text { (c) } x \in \mathrm{C}(R, A ; S) \text {. }
\end{aligned}
$$

Suponha que $x \in A$. Portanto, cxiste uma sequencia $\left\{z_{j}\right\}_{j=0}^{\infty} \subset A$ tal que $z_{j} \rightarrow x$, quando $j \rightarrow \infty$. Mas $A \subset \operatorname{Int}(U)$. Logo, cxistc $j_{0} \in \mathbb{N}$ tal que $z_{j} \in \operatorname{Int}(U)$, para todo $j \geq j_{0}$. Portanto, $y_{i} \in Z$, para toclo $i \geq i_{0}$. Ou seja, $y_{i} \in N_{1}$, para todo $i \geq i_{0}$. Isso implica que $x \in N_{1}$. Porćm, isso ó unla contradição.

Agora suponla quo $x \notin R$. Eutrino, $x \in \mathrm{C}(R, A ; S)$. Isto implica que $\omega(x) \subset A$. Tome $y \in \omega(x)$. Logo, cxistcm scquências $\left\{n_{k}\right\}_{k=1}^{\infty} \subset \mathbb{N}$ e $\left\{f^{n_{k}}(x)\right\}_{k=1}^{\infty}$ tais que $f^{n_{k}}(x) \rightarrow y$ e $n_{k} \rightarrow \infty$, quando $k \rightarrow \infty$. Como $A \subset \operatorname{Int}(U)$, existe $k_{0} \in \mathbb{N}$ tal que

$$
f^{n_{k}}(x) \in \operatorname{Int}(U) \subset Z \subset N_{1} \text { para todo } k \geq k_{0}
$$


Fixc $\bar{k} \geq k_{0}$. Então $f^{n_{k}}(x) \in \operatorname{Int}(U)$. Por outro lado, $f^{n_{k}}(x) \in \operatorname{cl}\left(N_{2} \backslash N_{1}\right)$, pois $x \in \operatorname{Inv}\left(\operatorname{cl}\left(N_{2} \backslash N_{1}\right)\right)$. Logo, existe uma scquência $\left\{z_{i}\right\}_{i=1}^{\infty} \subset N_{2} \backslash N_{1}$ tal que $z_{i} \rightarrow f^{n_{k}}(x)$, quando $i \rightarrow \infty$. Novanmente, existec $i_{0} \in \mathbb{N}$ tal cluce $z_{i} \in \operatorname{Int}(U) \subset N_{\mathrm{l}}$, para todo $i \geq i_{0}$. O que é uma contradição, pois $\left\{z_{i}\right\}_{i=1}^{\infty} \subset N_{2} \backslash N_{1}$. Portanto, $x \in R$.

Mostremos que $R=\operatorname{Inv}\left(\operatorname{cl}\left(N_{2} \backslash N_{1}\right)\right) \subset \operatorname{Int}\left(N_{2} \backslash N_{1}\right)$. Seja $x \in R$. Logo, $x \in S$. Conı $x \in S \subset \operatorname{Int}\left(N_{2} \backslash N_{0}\right)$. Portanto, existe $r_{0}>0$ tall que $B\left(x, r_{0}\right) \subset N_{2} \backslash N_{0}$. Definimos

$$
r:=\min \left\{\frac{r_{0}}{2}, \frac{\varepsilon}{2}\right\}
$$

e considere $y \in B(x, r)$. Portanto, $y \in B\left(x, r_{0}\right) \subset N_{2} \backslash N_{0}$. Resta provar que $y \notin \operatorname{cl}(Z)$. Suponla quc $y \in \operatorname{cl}(Z)$. Logo, existe uma sequência $\left\{y_{k}\right\}_{k=0}^{\infty} \subset Z$ tal que $y_{k} \rightarrow y$, quando $k \rightarrow \infty$. Utilizando argumcutos análogos aos anteriormente, temos que dist $\left(y_{k}, R\right)>\varepsilon_{0}$. Logo, $\operatorname{dist}(y, R) \geq \varepsilon_{0}$. Assim,

$$
\varepsilon_{0}<\operatorname{dist}(y, R) \leq \operatorname{dist}(y, x)<r \leq \frac{\varepsilon_{0}}{2},
$$

c isso é unna contradição. Portanto, $y \notin N_{1}$. Assinn,

$$
B(x, r) \subset N_{2} \backslash N_{1} \text {. }
$$

Desta maneira terminamos a verificação do item 1 da Definição 1.9 .

Passemos a demonstração do itenn 2 da definição de par-índice. Primeiramente, destaquemos que

$$
f(Z) \cap N_{2} \subset Z
$$

De fato, tome $y \in Z$ tal que $f(y) \in N_{2}$. Então $y=f^{n}(x)$, com $x \in Z$. Ou scja, $x \in U$ e $\left\{x, f(x), \ldots, f^{n}(x)\right\} \subset N_{2}$. Logo, $f^{n+1}(x)=f(y) \in N_{2}$. Logo, $\left\{x, f(x), \ldots, f^{\prime \prime}(x), f^{n+1}(x)\right\} \subset N_{2}$. Portanto, $f(y) \in Z$.

Vamos usar (2.2) para mostrar que $N_{1}$ é positivamcnte invariante com relação a $N_{2}$, isto ć,

$$
f\left(N_{1}\right) \cap N_{2} \subset N_{1}
$$

Tome $y \in f(\operatorname{cl}(Z))$. Então $y=f(z)$, com $z \in \operatorname{cl}(Z)$. Logo, existe uma scquência $\left\{z_{k}\right\}_{k=0}^{\infty} \subset Z$ tal que $z_{k} \rightarrow z$, quiando $k \rightarrow \infty$. Considcremos dois casos. Suponha que existe $k_{0} \in \mathbb{N}$ tal que $f\left(z_{k}\right) \in N_{2}$, para todo $k \geq k_{0}$. Usando (2.2), concluímos 
que $f\left(z_{k}\right) \in Z$, para torlo $k \geq k_{0}$. Como $y=f(z)$ ć limite da sequência $\left\{f\left(z_{k}\right)\right\}_{k=0}^{\infty}$, tomos que $y \in \mathrm{cl}(Z)$. O que implica que $y \in N_{1}$.

Suponha que existe uma sequência $\left\{n_{k}\right\}_{k=1}^{\infty} \in \mathbb{N}$ tal que $f\left(z_{n_{k}}\right) \notin N_{2}$. Temos que $f\left(z_{n_{k}}\right) \rightarrow f(z)$, quando $k \rightarrow \infty$, c $z_{n_{k}} \in N_{2}$, para todo $n_{k}$. Portanto,

$$
z_{n n_{k}} \in N_{2} \subset f\left(z_{n_{k}}\right) \notin N_{2} \text {. }
$$

Como $\left(N_{2}, N_{0}\right)$ é uın par-índice parä $S$, concluímos quc

$$
z_{u_{k}} \in N_{0} \text {. }
$$

Como $z$ é o limite da sequência $\left\{z_{n_{k}}\right\}_{k=1}^{\infty}$ c $N_{0}$ é um conjunto compacto, temos que

$$
z \in N_{0} \text {. }
$$

Logo,

$$
z \in N_{0} \text { e } f(z) \in N_{2} .
$$

Novamente, o fato de $\left(N_{2}, N_{0}\right)$ ser par-índicc nos leva a concluir que $y \in N_{1}$. Portanto, em qualquer dos dois casos obtemos que $y \in N_{1}$.

Para a verificação do tcrceiro item da Definição 1.9 , basta notar que

$$
N_{2} \backslash N_{1} \subset N_{2} \backslash N_{0}
$$

Logo,

$$
f\left(N_{2} \backslash N_{1}\right) \subset f\left(N_{2} \backslash N_{0}\right) \subset N_{2}
$$

Resta provar que $\left(N_{2}, N_{1}\right)$ ć un par-índice regular. Primciramente note que

$$
f\left(N_{1}\right) \backslash N_{2} \subset f\left(N_{0}\right) \backslash N_{2}
$$

De fato, tome $x \in N_{1}=N_{0} \cup \operatorname{cl}(Z)$. Então $f(x) \in f\left(N_{0}\right) \cup f(\operatorname{cl}(Z))$. Suponha que $f(x) \in f(\operatorname{cl}(Z))$. Então, existe $z \in Z$ tal que $f(x)=f(z)$. Logo, existe uma sequência $\left\{z_{k}\right\}_{k=1}^{\infty} \subset Z$ tal quc $z_{k} \rightarrow z$. Portanto, $f\left(z_{k}\right) \rightarrow f(z)$, quando $k \rightarrow \infty$. Novamentc dividircmos a clemonstração em duas situações. Suponha que existc $k_{0} \in \mathbb{N}$ tal que $f\left(z_{k}\right) \in N_{2}$, para todo $k \geq k_{0}$. Por (2.2), temos que $f\left(z_{k}\right) \in Z$. Assim, $\left\{f\left(z_{k}\right)\right\}_{k=1}^{\infty}$ é uma seq̣ıchncia cm $Z$ tal que $f\left(z_{k}\right) \rightarrow f(z)=y$. Portanto, $y \in \operatorname{cl}(Z) \subset N_{2}$. O que é unla contradição. Agora, nos resta a seguinte possibilidadc: existem sequências $\left\{z_{n_{k}}\right\}_{k=1}^{\infty} \subset Z$ c $\left\{n_{k}\right\}_{k=1}^{\infty} \subset N$ tais que

$$
f\left(z_{n_{k}}\right) \notin N_{2}, z_{n_{k}} \rightarrow z \mathrm{c} n_{k} \rightarrow \infty \text {, quando } k \rightarrow \infty \text {. }
$$


Coıno $\left(N_{2}, N_{0}\right)$ é par-índice, $z_{k} \in N_{2}$ e $f\left(z_{n_{k}}\right) \notin N_{2}$, temos que $f\left(z_{n_{k}}\right) \in N_{0}$. Portanto, $z \in N_{0}$ e $f(z) \notin N_{2}$, ou scja, $y=f(z) \in f\left(N_{0}\right)$. Logo,

$$
f\left(N_{1}\right) \subset f\left(N_{0}\right)
$$

Com isso provamos a inclusino (2.3). Logo,

$$
\operatorname{cl}\left(N_{2} \backslash N_{1}\right) \cap \operatorname{cl}\left(f\left(N_{1}\right) \backslash N_{2}\right) \subset \operatorname{cl}\left(N_{2} \backslash N_{0}\right) \cap \operatorname{cl}\left(f\left(N_{0}\right) \backslash N_{2}\right)=\emptyset .
$$

Concluínos, assim, que $\left(N_{2}, N_{1}\right)$ é par-inclice regular para $R$.

(ii) O par $\left(N_{1}, N_{0}\right)$ ć par-índice regular para $A$.

Para a prova do primciro item da dcfinição de par-índicc, tome $x \in A$. Logo, $x \in S=\operatorname{Inv}\left(N_{2} \backslash N_{0}\right)$. Portanto, cxistc urna sequência $\left\{x_{n}\right\}_{-\infty}^{\infty} \subset N_{2} \backslash N_{0}$ tal que $f\left(x_{n}\right)=x_{n+1}$ e $x_{0}=x$. Em particular, $x \in N_{2} \backslash N_{0}$. Desta forma, existe uma sequência $\left\{y_{k}\right\}_{k=0}^{\infty} \subset N_{2} \backslash N_{0}$ tal que $y_{k} \rightarrow x$, quando $k \rightarrow \infty$. Como $A$ é um conjunto atrator, cxistc $U \subset X$ tal que $A \subset U$. Portanto, $U$ é uma vizinhança para $x$. Logo, existe $k_{0} \in \mathbb{N}$ tal que $y_{k} \in U \subset N_{\mathrm{l}}$, para todo $k \geq k_{0}$. Assim, $y_{k} \in N_{\mathrm{l}} \backslash N_{0}$, para todo $k \geq k_{0}$. Portanto,

$$
x \in \mathrm{cl}\left(N_{\mathrm{l}} \backslash N_{0}\right)
$$

Usando o Teorema 2.5 itcm 2 , tcınos quc existe una scçuência $\left\{y_{n}\right\}_{-\infty}^{\infty} \subset A$ tal que $f\left(y_{n}\right)=y_{n+1}$ × $y_{0}=x$. Mas o argmucnto acima inostra que $\left\{y_{n}\right\}_{-\infty}^{\infty} \subset N_{1} \backslash N_{0}$. Portanto,

$$
x \in \operatorname{Inv}\left(\operatorname{cl}\left(N_{1} \backslash N_{0}\right)\right)
$$

Seja $x \in \operatorname{Inv}\left(\operatorname{cl}\left(N_{1} \backslash N_{0}\right)\right) \subset \operatorname{Inv}\left(\operatorname{cl}\left(N_{2} \backslash N_{0}\right)\right)=S$. Em particular, $x \in \operatorname{cl}\left(N_{1} \backslash N_{0}\right)$. Logo, cxistc una sequência $\left\{y_{k}\right\}_{k=0}^{\infty} \subset N_{1} \backslash N_{0}$ tal que $y_{k} \rightarrow x$, quando $k \rightarrow \infty$. Conno $x \in S$, lıa três possibilidades para o ponto $x$

$$
\begin{aligned}
& \text { (a) } x \in A, \\
& \text { (b) } x \in R \mathrm{e} \\
& \text { (c) } x \in \mathrm{C}(R, A ; S) \text {. }
\end{aligned}
$$

Suponlia que $x \in R$. Assim, existe $k_{0} \in \mathbb{N}$ tal que $y_{k} \in \operatorname{Int}\left(N_{2} \backslash N_{1}\right)$, para todo $k \geq k_{0}$. Ou seja, $y_{k} \notin N_{1}$, para todo $k \geq k_{0}$. O que é uma contradição.

Agora, suponha quc $x \in \mathrm{C}(R, A ; S)$. Então $\omega(x) \subset A$. Como $x \in S$, existe scquência $\left\{x_{-n}\right\}_{n=1}^{\infty} \subset S$ tal que $\int\left(x_{-n}\right)=x_{-n+1}$ c $x_{0}=x$. Como $S$ ć uın conjunto 
compacto, existc uma subsequêcia $\left\{x_{-n_{k}}\right\}$ tal que $x_{-n_{k}} \rightarrow \bar{y}$, quando $n \rightarrow \infty$. Pelo Teorcma 2.5, itcm 3 (d) $\vec{y} \in R$. Logo, cxiste $n_{k_{0}} \in \mathbb{N}$ tal que $x_{-n_{k}} \in \operatorname{Int}\left(N_{2} \backslash N_{1}\right)$, para todo $n_{k} \geq n_{k_{0}}$. Fixe $n_{\bar{k}} \geq n_{k_{0}}$. Enttão, $x_{-n_{k}} \in \operatorname{Int}\left(N_{2} \backslash N_{1}\right)$ e $x_{-n_{k}} \in \operatorname{Inv}\left(\operatorname{cl}\left(N_{1} \backslash N_{0}\right)\right)$. $\mathrm{O}$ (que é unna contradição. Portanto, a lunica possibilidarle ć

$$
x \in A \text {. }
$$

Mostremos que $A=\operatorname{Inv}\left(\operatorname{cl}\left(N_{1} \backslash N_{0}\right)\right) \subset \operatorname{Int}\left(N_{\mathrm{l}} \backslash N_{0}\right)$. Com este intuito, tome $x \in A$. Couno $A \subset \operatorname{Int}(U) \subset Z 0 x \in S$, outino $x \in \operatorname{Int}\left(N_{2} \backslash N_{1}\right)$. Ou soja, cxistc $r_{0}>0$ tal que

$$
x \in B\left(x, r_{0}\right) \cap \operatorname{Int}(U) \subset \operatorname{Int}\left(N_{2} \backslash N_{0}\right) \cap Z \subset Z \backslash N_{0} \subset N_{1} \backslash N_{0} .
$$

Defina $B:=B\left(x, r_{0}\right) \cap \operatorname{Iut}(U)$. Note que $B$ é um aberto tal que $x \in B$ e $B \subset N_{\mathrm{l}} \backslash N_{0}$.

A prova de que $N_{\mathrm{l}}$ é positivamentc invariante com relação a $N_{2}$ é análoga a prova fcita em (i).

Para o terceiro item, primeiramente, note que

$$
N_{\mathrm{I}} \backslash N_{0} \subset \operatorname{cl}(Z) \backslash N_{0}
$$

Tome $y \in f\left(N_{1} \backslash N_{0}\right)$. Logo, $y=f(x)$, com $x \in N_{\mathrm{l}} \backslash N_{0}$. Portanto, $x \in \operatorname{cl}(Z) \subset N_{2}$ e $x \notin N_{0}$. Logo, $x \in N_{2} \backslash N_{0}$. Assim, $y=f(x) \in f\left(N_{2} \backslash N_{0}\right) \subset N_{2}$. Ou seja, $y \in f\left(N_{1}\right) \cap N_{2}$. Mas, como $\left(N_{2}, N_{1}\right)$ é par-indice para $R$, temos que $f\left(N_{1}\right) \cap N_{2} \subset N_{1}$. Logo, $y \in N_{1}$. O que implica que $N_{0}$ ć o conjunto saída para $\left(N_{1}, N_{0}\right)$.

Para cncerrar a prova cleste resultiado verifiquemos a inclusão a seguir

$$
f\left(N_{0}\right) \backslash N_{1} \subset f\left(N_{0}\right) \backslash N_{2}
$$

Tonc $y \in f\left(N_{0}\right) \backslash N_{1}$. Entĩo existe $x \in N_{0}$ tial que $y=f(x)$. Suponha que $f(x) \in N_{2}$. Logo, $x \in N_{0}$ tal rute $f(x) \in N_{2}$. Conto $\left(N_{2}, N_{0}\right)$ ć par-índice para $S$, $f(x) \in N_{0}$. Portanto, seguc que

$$
y \in N_{0} \cup \operatorname{cl}(Z)=N_{1} .
$$

O que gera una contradlição. Logo,

$$
y \in f\left(N_{0}\right) \backslash N_{2} \text {. }
$$

Decorre facilucute de (2.4) que $\left(N_{1}, N_{0}\right)$ ó par-ínclice regular para $A$. 
Observação 2.12. Nole çuc o trio-índice $\left(N_{2}, N_{1}, N_{0}\right)$ definido na demonstração da Proposição 2.11 satisfà: $N_{0} \subset N_{2}, N_{0} \subset N_{1}$ c $N_{1} \subset N_{2}$.

A partir da existência de um trio-índice para unna decomposição atratora-repulsora construiremos uma scquência longa envolvendo os índices de $S, A$ e $R$. No que segue a colıomologia dos índices de Conley serão calculados usando coeficientes sobre uln corpo.

Proposição 2.13. Se $(A, R)$ é uma decomposiçãa atratora-repulsora para o conjunto invariante isolado $S$, obtem-se a seguinte sequência longa de espaços vetoriais $e$ automorfismos

$$
\cdots \stackrel{\delta^{k-1}}{\longrightarrow} \operatorname{Con}^{k}(R) \rightarrow \operatorname{Con}^{k}(S) \rightarrow \operatorname{Con}^{k}(A) \stackrel{\delta^{k}}{\rightarrow} \operatorname{Con}^{k+1}(R) \rightarrow \cdots
$$

Demonstração. Scja $\left(N_{2}, N_{1}, N_{0}\right)$ o um trio-índice regular para $(A, R)$ construído na demonstração da Proposição 2.11. Para facilitar a exposição iremos usar a scguinte notação:

$$
\begin{aligned}
M_{0}^{0}:=N_{0} \cup f\left(N_{0}\right), \\
M_{1}^{0}:=N_{1} \cup f\left(N_{0}\right), \\
M_{2}^{0}:=N_{2} \cup f\left(N_{0}\right) \mathrm{c} \\
M_{2}^{1}:=N_{2} \cup f\left(N_{1}\right) .
\end{aligned}
$$

Assim, faz sentido definir as seguintes aplicações:

$$
\begin{gathered}
\iota:\left(N_{1}, N_{0}\right) \rightarrow\left(N_{2}, N_{0}\right), \text { dada por } \iota(x)=x, \\
\rho:\left(N_{2}, N_{0}\right) \rightarrow\left(N_{2}, N_{1}\right), \text { dada por } \rho(x)=x, \\
\tilde{\iota}:\left(M_{1}^{0}, M_{0}^{0}\right) \rightarrow\left(M_{2}^{0}, M_{0}^{0}\right), \text { dacla por } \tilde{\iota}(x)=x, \\
\tilde{\rho}:\left(M_{2}^{0}, M_{0}^{0}\right) \rightarrow\left(M_{2}^{1}, M_{1}^{1}\right) \text {, dada por } \tilde{\rho}(x)=x .
\end{gathered}
$$

Destc modo, o diagrama 


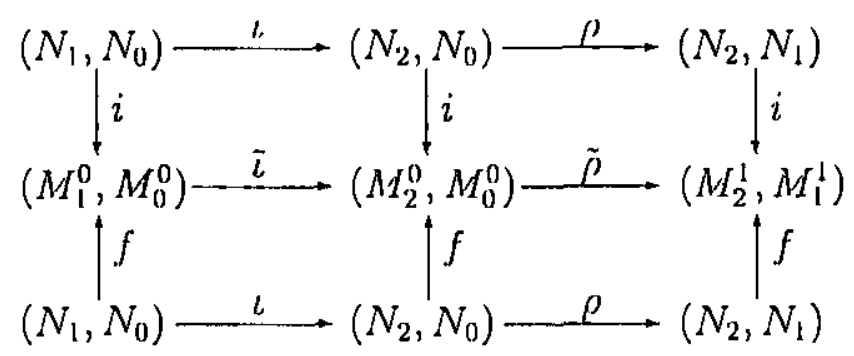

ć comutativo, onde $i$ denota aplicação inclusão. Passando ao nível de cocadeia, obtcmos o seguinte diagrama conntativo.

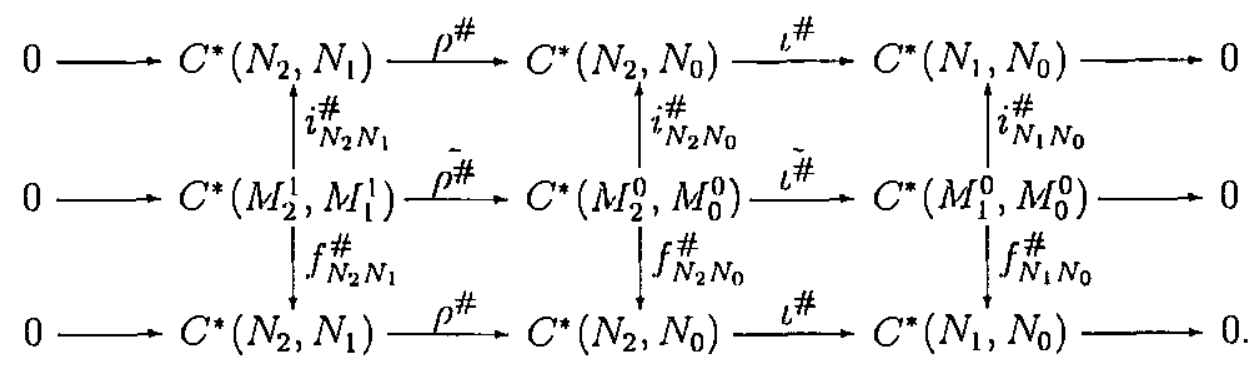

Como $i_{N_{2} N_{1}}^{\#}, i_{N_{1} N_{0}}^{\#}$ c $i_{N_{2} N_{0}}^{\#}$ são isomorfismos c lembrando que $f_{N_{2} N_{1}}^{\#}=f^{\#}$ 。 $\left(i_{N_{2} N_{1}}^{\#}\right)^{-1}, f_{N_{1} N_{0}}^{\#}=f^{\#} \circ\left(i_{N_{1} N_{0}}^{\#}\right)^{-1}$ e $f_{N_{2} N_{0}}^{\#}=f^{\#} \circ\left(i_{N_{2} N_{0}}^{\#}\right)^{-1}$, obtemos o seguinte diagrama comutativo

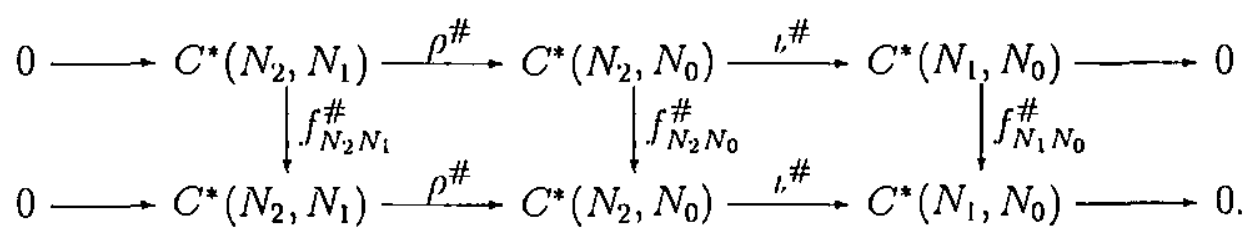

Passaundo ao nível de cohomologia c rcalizando a Redução de Leray obtemos novanente o digranıa conutativo de seçuchucias cxatas longas:

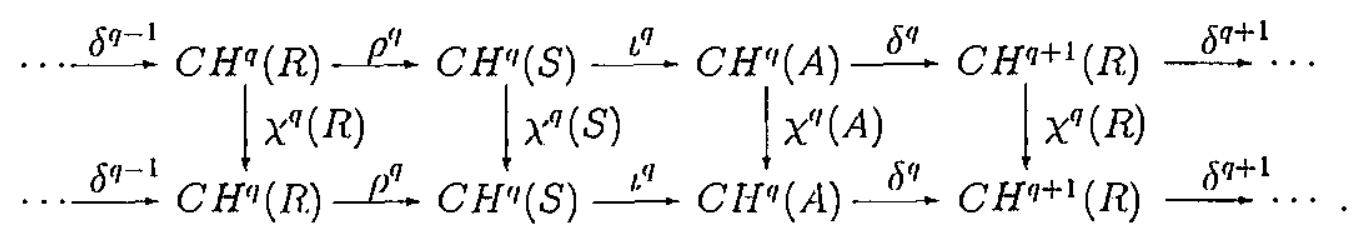


De modo mais simplificado a sequência anterior será denotada por

$$
\cdots \stackrel{\delta^{k-1}}{\longrightarrow} \operatorname{Con}^{k}(R) \rightarrow \operatorname{Con}^{k}(S) \rightarrow \operatorname{Con}^{k}(A) \stackrel{\delta^{k}}{\rightarrow} \operatorname{Con}^{k+1}(R) \rightarrow \cdots
$$

Com isso a demonstração estrí concluída.

A Propriedade de Aditividade nos fornece a seguinte inforınação sobre a aplicação $\delta^{*}$ do teorema anterior para o caso $S=A \cup R$.

Corolário 2.14. Sejam $S$ um conjunto invariante isolado e $(A, R)$ sua decomposição atratora-repulsora. Se $S=A \cup R$, entäo, para todo $q \in \mathbb{Z}, \delta^{q}=0$, $C H^{\prime \prime}(S) \cong C H^{q}(A) \times C H^{q}(R)$ e $\chi^{\prime \prime}(S)$ é conjugada a $\chi^{q}(A) \times \chi^{q}(R)$.

DemonstraÇÃo. A Propriedade de Aditividade nos leva a concluir facilmente que, para todo $q \in \mathbb{Z}, \operatorname{Con}^{q}(S) \cong \operatorname{Con}^{q}(A) \times \operatorname{Con}^{q}(R)$.

Mostremos que $\delta^{\prime}=0$. Tome a seçucucia cxata como na demonstração do tcorema anterior

$$
\ldots \stackrel{\delta^{q-1}}{\longrightarrow} \operatorname{Con}^{q}(R) \stackrel{\rho^{q}}{\longrightarrow} \operatorname{Con}^{q}(S) \stackrel{\iota^{q}}{\longrightarrow} \operatorname{Con}^{q}(A) \stackrel{\delta^{q}}{\longrightarrow} \operatorname{Con}^{q+1}(R) \stackrel{\delta^{q+1}}{\longrightarrow} \cdots
$$

Logo, $\operatorname{Im}\left(\delta^{q}\right)=\operatorname{ker}\left(\rho^{q}\right)$. Conı $\rho^{\#}$ é unna aplicação injetora, existc

$$
\varphi^{\#}: C^{*}\left(N_{2}, N_{0}\right) \rightarrow C^{*}\left(N_{2}, N_{1}\right)
$$

tal que, $\varphi^{\#}$ é uma inversa à esquerda para $\rho^{\#}$. Ou scja,

$$
\varphi^{\#} \circ \rho^{\#}=\mathrm{Id}_{N_{2} N_{1}} .
$$

Passando ao nível de colıomologia obtemos

$$
\varphi^{\eta} \circ \rho^{q}=\mathrm{Id}_{R}
$$

Logo, $\varphi^{\eta}$ é uma aplicação sobrcjetora c $\rho^{\eta}$ ć uuna aj)licação injetora. Portanto, $\operatorname{ker}\left(\rho^{q}\right)=\{0\}$.

O resultado que scgue estabelece a contra positiva do corolário anterior.

Corolário 2.15. Sejam $S$ um conjunto invariante isolado $e(A, R)$ sua decomposição atratora-repnlsora. Se $\delta^{q} \neq 0$, ou $C H^{q}(S)$ não é isomorfo a $C H^{q}(A) \times$ $C H^{\prime \prime}(R)$, ou a aplicação $\chi^{q}(S)$ nüo é conjugada a $\chi^{\prime \prime}(A) \times \chi^{\prime \prime}(R)$, então $S \neq A \cup R$. Em particular, $\mathrm{C}(R, A ; S) \neq \emptyset$. 
Finalizamos o capítılo ilustrando alguns dos conceitos vistos.

Exemplo 2.16. Scjaun $X=S^{2}=\mathbb{R}^{2} \cup\{\infty\}$ c $f: X \rightarrow X$ uma aplicação contínua que aplica dois retângulos $N_{1}$ e $N_{2}$ como na Fignra 2.1. $\operatorname{Sejam} A=\operatorname{Inv}\left(N_{1}\right)$,

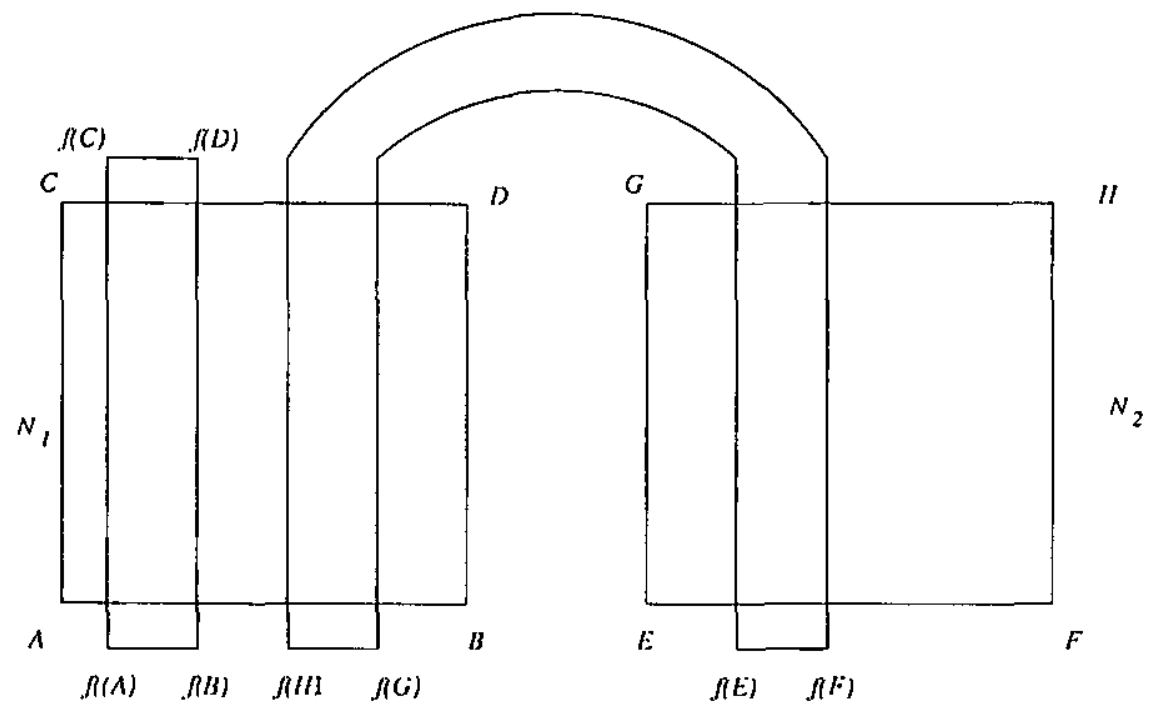

Figura 2.1: Uin par atrator-repulsor.

$R=\operatorname{Inv}\left(N_{2}\right)$ e $S=\operatorname{Inv}\left(N_{1} \cup N_{2}\right)$. Bascada na definição geométrica descrita na Figura 2.1, ircmos justificar que $(A, R)$ e unıa decomposição atratora-repulsora para $S$. Mostremos que $A$ é uın atrator cm $S$. Note que $N_{1}$ é uma vizinhança de $A$. Toine $x \in A=\operatorname{Inv}\left(N_{1}\right)$, então existe uma sequência $\left\{x_{n}\right\}_{-\infty}^{\infty} \subset N_{1} \cap S$ tal que $f\left(x_{n}\right)=x_{n+1}$ c $x_{0}=x$. Tomanclo sequências $\left\{n_{k}\right\}_{k=0}^{\infty} \subset \mathbb{N}$ e $\left\{y_{k}\right\}_{k=0}^{\infty} \subset N_{1} \cap S$ tais que $n_{k}=k$ e $\eta_{k}=x_{-k}$, temos que $n_{k} \rightarrow \infty$, quando $k \rightarrow \infty \mathrm{e}$

$$
f^{n_{k}}\left(y_{k}\right)=f^{k}\left(x_{-k}\right)=x
$$

Portanto,

$$
x \in \omega\left(N_{1} \cap S\right) \text {. }
$$

Agora, $\omega\left(N_{1} \cap S\right) \subset N_{1}$ é um conjunto invariante. Logo, $\omega\left(N_{1} \cap S\right) \subset A$. Portanto, $A$ ci lin conjunto atrator.

A presentamos, também, a prova de que $R$ é um conjunto repulsor para $S$. Tome $x \in R=\operatorname{Inv}\left(N_{2}\right)$ e suponlia que $\omega(x) \cap A \neq \emptyset$. Scja $y \in \omega(x) \cap A$. Logo, $y \in A$. Como $y \in \omega(x)$, existcm sequências $\left\{n_{k}\right\}_{k=0}^{\infty}$ c $\left\{y_{k}\right\}_{k=0}^{\infty}$ tais que $n_{k} \rightarrow \infty$ e $f^{n_{k}}\left(y_{k}\right) \rightarrow y$, quando $k \rightarrow \infty$. Como $x \in N_{2}$, cutino $f^{\mu_{k}}(x) \in N_{2}$, para todo $n_{k}$. Sendo $N_{2}$ um 
conjunto compacto c $y$ linite da sequência $\left\{f^{n_{k}}(x)\right\}$, temos que $y \in N_{2}$. O que ć muna contradição. Para finali»ar, tome $x \in S=\operatorname{Inv}\left(N_{1} \cup N_{2}\right)$ e suponha que $\omega(x) \cap A=\emptyset$. Como $x \in \operatorname{Inv}\left(N_{1} \cup N_{2}\right)$, existe una seculuencia $\left\{x_{n}\right\}_{-\infty}^{\infty} \subset N_{1} \cup N_{2}$ tal que $f\left(x_{n}\right)=x_{n+1}$ c $x_{0}=x$. Suponha que existe $n_{0} \in \mathbb{Z}$ tal que $x_{n_{0}} \in N_{1}$. Como $A=\omega\left(N_{1} \cap S\right)$, cutão $x_{n_{0}} \in N_{1} \cap S$. Ou seja, $\omega\left(x_{n_{0}}\right) \in \omega\left(N_{1} \cap S\right)$. Lógo, $\omega\left(x_{n_{n_{0}}}\right) \cap A \neq \emptyset$. Senclo $\omega(x)=\omega\left(x_{n_{0}}\right)$, tomos que $\omega(x) \cap A \neq \emptyset$. Isto é uma contradição. Portanto, a sequência $\left\{x_{n}\right\}_{-\infty}^{\infty}$ está inteiramente contida em $N_{2}$. O que significin que $x \in \operatorname{Inv}\left(N_{2}\right)$.

Passemos agora ao calculo do índice de Conley para $A, R$ e $S$. É fácil ver que $N_{\mathrm{I}} \cap f\left(N_{1}\right) \cap f^{-1}\left(N_{1}\right) \subset \operatorname{Int}\left(N_{1}\right)$. Logo, $N_{\mathrm{I}}$ c bloco isolantc. Seja $L_{1}$ o conjunto de saída innediato de $N_{1}$, isto é, $L_{1}:=N_{1}^{-}$. Na Figura 2.2 temos a representação geonétrica do par $\left(N_{1}, L_{1}\right)$. Procedendo cono $\mathrm{cm}$ exemplos anteriores, é fácil ver quc

$$
H^{q}\left(N_{1}, L_{1}\right)= \begin{cases}\mathbb{Q}, & \text { se } q=1 \\ 0, & \text { caso contrário }\end{cases}
$$

Alćnı disso,

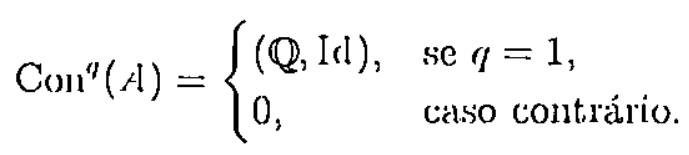

Analogamente,

$$
\operatorname{Con}^{q}(R)= \begin{cases}(\mathbb{Q}, I \mathrm{I}), & \text { se } q=1, \\ 0, & \text { caso contrário. }\end{cases}
$$

Finalucnte, voltemos nossa atcuçĩo para $S$. Note que $N_{\mathrm{I}} \cup N_{2}$ é bloco isolante para $S$. Torne $N=N_{1} \cup N_{2}$ c $L=\left(N_{1} \cup N_{2}\right)^{-}$. Logo, $(N, L)$ é um par-índice para $S$. Na Figura 2.2, encontra-se uma represcntação para o par $(N, L)$. O conjunto $L$ é a união de $L_{1}$ e $L_{2}$.

Novancute temos que

$$
H^{\prime}(N, L)= \begin{cases}\mathbb{Q}^{3}, & \text { se } q=1 \\ 0, & \text { caso contrário. }\end{cases}
$$

Um cálculo simples mostra que uma representaçĩo inatricial para $\left(f_{N L}^{1}\right)^{\prime}$ é

$$
\left(\begin{array}{cc}
1 & -1 \\
0 & 1
\end{array}\right)
$$




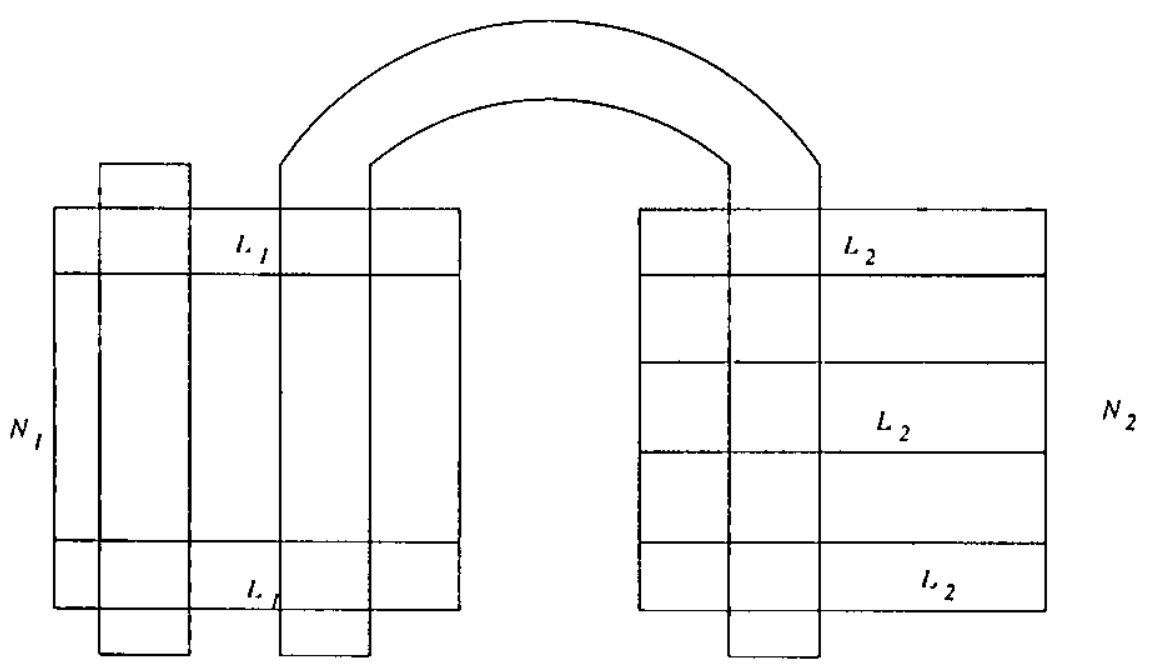

Figura 2.2: Un par-índice para $S$.

Logo,

$$
\operatorname{Con}^{q}(S)= \begin{cases}\left(\mathbb{Q} \times \mathbb{Q},\left(\begin{array}{cc}
1 & -1 \\
0 & 1
\end{array}\right)\right), & \text { se } q=1, \\
0, & \text { caso contrário. }\end{cases}
$$

Neste exemplo, lá existência de ólbitàs de conexão. No final clo próximo capítulo mostraremos resultarlos mais gerais que também implicam a existência de órbitas de concxão. 


\section{Capítulo 3}

\section{Decomposição de Morse e Par de Matrizes de Conexão}

Nestc capítulo, finalınente aprescutaremos a definição de clecomposição de Morse para um conjunto invariante isolaclo e de par cle unatrizes de conexão associado a uma decomposição de Morse. Para csse fim, torna-se necessário o conceito de filtração índice que ć aprescutado tta Seção 3.1. O par de matrizes de concxão é apresentado na Seção 3.2. As refcrêucias básicas cleste capítulo são [9] e [10]. Novamente, a colınologia dos índices de Conley scrão calculados nsando coeficientes sobre um corpo.

\subsection{Decomposição de Morse e Filtração Índice}

Nesta seção introduzircınos nma relação de orden parcial com o intuito de "ordenar" os conjuntos atratorcs o repulsores que unı conjunto invariante isolado $S$ pode apresentar.

Definição 3.1. Seja $\mathcal{P}$ unn conjunto fintito. Dizemos que < é uma relação de ordem parcial $\mathrm{em} \mathcal{P}$, se:

1. $p<p$ nunca ocorre,

2. se $p, q$ e $r \in \mathcal{P}$, sãn tais que. $p<q$ e $q<r$, entãn $p<r$.

Observação 3.2. Sc a rolaşão cstabelccida anteriornente satisfizer à seguinte conclição: sc $p \neq q$, cutĩio ou $p<q$, ou $q<p$, dizcınos quc < é uma relação de ordem 
total ell $\mathcal{P}$.

Sc $\mathcal{P}$ ć um conjunto finito munido de uma orden parcial < temos condiçōes de definir intervalos em $\mathcal{P}$.

Definição 3.3. Sejam $(\mathcal{P},<)$ um conjunulo finito e $<$ uma ordem parcial. Um sub)canjunto $I \subset \mathcal{P}$ é um intervalo cm $\mathcal{P}$, se $p<r<q$, corn $p, q \in I$, então $r \in I$.

O conjunto dos intervalos em $\mathcal{P}$ ć denotado por $\mathcal{I}(\mathcal{P},<)$. Muitas vezes usaremos a notação simplificada $\mathcal{I}$.

Definição 3.4. Seja $(\mathcal{P},<)$ um. conjunto funito munido de uma ordem parcial. Um intervalo $I \in \mathcal{I}$ é urn intervalo atrator, se $r<q$, com $q \in I$ implicar $r \in I$.

Denotannos o conjunto dos intervalos atratores cm $(\mathcal{P},<)$ por $\mathcal{A}(\mathcal{P},<)$. O mesmo ć denotado, de manteira simplificada, por $\mathcal{A}$.

Definição 3.5. Uma coleção do inblervalos cm $\mathcal{P},\left(I_{1}, I_{2}, \ldots, I_{n}\right)$ dois a dois disjuntos é uma n-upla adjaccnte ern $\mathcal{P}$, se satisfaz:

1. $I_{1} \cup I_{2} \cup \ldots \cup I_{n-1} \cup I_{n} \in \mathcal{I}$,

2. se $p \in I_{i}$ e $q \in I_{j}, \operatorname{com} i<j$, cntãaq $\not<$.

A união $\left(I_{1}, I_{2}, \ldots, I_{n}\right)$ scrá denotada simplesinente por $I_{1} I_{2} \ldots I_{n}$. Denota-se o conjunto das $n$-uplas adjacentes em $\mathcal{P}$ por $\mathcal{I}_{n}(\mathcal{P},<)$. Ou apenas por $\mathcal{I}_{n}$.

Devidamente definidos ordem parcial $\mathrm{mm}$ um conjunto finito $\mathcal{P}$, intervalos em $\mathcal{P}$, pockemos definir decomposição de Morse parn unı conjunto invariante isolado $S$.

Definição 3.6. Sejam $S$ um conjunuto invvariarate isolado, $(\mathcal{P},<)$ um conjunto finito parcialmente ordenada. Uma dcconıposição de Morse para $S$ é uma coleção de conjuntos campactos invariantes isolados

$$
\mathcal{M}(\mathcal{P},<):=\left\{M_{p} \subset S \mid p \in \mathcal{P}\right\}
$$

cam a propriedade que se

$x \in S \backslash\left(\bigcup_{p \in \mathcal{P}} M_{p}\right)$, entio exristem $p, q \in \mathcal{P}$ tais que $q<p \quad$ e $x \in \mathrm{C}\left(M_{p}, M_{q} ; S\right)$, 
onde

$$
\begin{gathered}
\mathrm{C}\left(M_{p}, M_{q} ; S\right)=\left\{x \in S \mid \text { existem } q<p \in I \text { com a propriedade } \omega(x) \subset M_{q}\right. \\
\text { e pura toda sequência } \left.\left\{n_{j}\right\}_{j=0}^{\infty} \subset \mathbb{N} \text { tal que } x_{-n_{k}} \rightarrow \bar{y} \text {, então } \bar{y} \in M_{p}\right\} .
\end{gathered}
$$

Os conjuntos $M_{p}$ são chamados de conjuntos de Morse. Muitas vezes denotaremos unua clecomposição de Morse para $S$ cle mancira curta por $\mathcal{M}$.

Exemplo 3.7. Un cxcmplo simples de unla decomposição de Morse ć a decomposição atratola-repulsora de mu conjunto invariante isolado. De fato, se $(A, R)$ é una decomposição atratora-repulsora de un conjunto invariante isolado, basta tomar $\mathcal{P}=\{1,2\}$, com a relação $1<2$. chame $M_{1}=A$ e $M_{2}=R$. Então $\left\{M_{1}, M_{2}\right\}$ ć uma decomposição de Morse para $S$.

Note que dado um conjuuto finito $\mathcal{P}$ podcrianos definir em $\mathcal{P}$ duas ou mais relaçõcs de ordem parcial < e <', e desta maneira iríamos obter duas ou mais decomposições de Morse para 111 mesmo conjunto invariante isolado $S$.

Note também que dado mua coleção de conjuntos de Morse $\mathcal{M}(\mathcal{P},<)$, poderíamos dar a $\mathcal{P}$ uma ordem parcial difereute de modo que as propriedades da Definição 3.6 continuem sendo válidas. Além clisso, $f$ define uma ordem parcial sobre os conjuntos cahmada ordem f-definida. Qualquer outra ordem parcial é uma extensão desta ordem $f$-definida c é chamada admissível.

Dado um intervalo $I \in \mathcal{I}(\mathcal{P},<)$, iremos defiuir $\mathrm{mm}$ conjunto invariante associado a $I$,

Definição 3.8. Sejam $(\mathcal{P},<)$ un conjinutulo fintito parcialmenle ordenado e $I \in \mathcal{I}$ um inlervalo em $\mathcal{P}$. Definimos

$$
M_{l}:=\left(\bigcup_{p \in I} M_{p}\right) \bigcup\left(\bigcup_{p, q \in I} \mathrm{C}\left(M_{p}, M_{q} ; S\right)\right) .
$$

O conceito de filtração índice, aprescutiaclo a seguir, generaliza o conceito de trio-índice apresentado no capítulo anterior.

Definiçāo 3.9. Sejam $S$ un conjunto invontiantc isolado, $(\mathcal{P},<)$ um conjunto finito parcialmente ordenado e $\mathcal{M}$ uma decomposição de Morse pura $S$. Uma coleção de conjuntos compactos

$$
\mathcal{N}(\mathcal{P},<):=\{N(I) \mid I \in \mathcal{A}\}
$$


é clıamada de filtração índice regular porn decomposição de Morse $\mathcal{M}$, se satisfaz:

1. para cada. $I \in \mathcal{A},(N(I), N(\emptyset))$ é um par-índicc regular para $M_{1}$,

2. para todo $I, J \in \mathcal{A}$, tcmos que $N(I) \cap N(J)=N(I \cap J)$ e $N(I) \cup N(J)=$ $N(I \cup J)$.

Observação 3.10. Scjamn $\mathcal{M}(\mathcal{P},<)$ nma decomposição de Morse para um conjunto invariante isolado $S$ e $\mathcal{N}(\mathcal{P},<)$ mma filtração ínclicc para $\mathcal{M}$. Segue de condição 2 dat Definição 3.9 que parán torlo $J \in \mathcal{A}, N(\emptyset) \subset N(J)$.

Uma filtração índice podc ser utilizada para determinar um par-índice para outros conjuntos invariantes isolados. Este resultado pode ser encontrado em Richeson [९].

Proposiçāo 3.11. Sejam $\mathcal{M}(\mathcal{P},<)$ uma decomposiçãa de Morse para o conjunto invariante isalado $S$ e $\mathcal{N}(\mathcal{P},<)$ filtração indicc para $\mathcal{M}$. Considere $I, J \in \mathcal{I}$ dois intervalos atratores, tais que $J \subset I$. Entiăo $(N(I), N(J))$ é um par-indice para $M_{\Lambda J}$.

DemonstraçÄo. Temos de verificar as condições da Definição 1.9. Seja $x \in$ $\operatorname{Inv}(\operatorname{cl}(N(I) \backslash N(J)))$. En particular, $x \in \operatorname{cl}(N(I) \backslash N(J))$. Logo, existc uma sequência $\left\{x_{k}\right\}_{k=0}^{\infty} \subset N(I) \backslash N(. J)$ tial que $x_{k} \rightarrow x$, quando $k \rightarrow \infty$. Então,

$$
x \in M_{I}=\left(\bigcup_{p \in I}\right) \cup\left(\bigcup_{p, q \in I} \mathrm{C}\left(M_{p}, M_{q} ; M_{I}\right)\right) .
$$

Supoulia que $x \in \mathrm{C}\left(M_{p}, M_{\eta} ; M_{l}\right)$, com $p, q \in I$ < $q<p$. Portanto, $\omega(x) \subset M_{q}$ c para toda sequência $\left\{n_{j}\right\}_{j=0}^{\infty} \subset \mathbb{N}$ tal que $x_{-n_{k}} \rightarrow \bar{y}$. Pelo Teorema 2.5, temos que $\ddot{y} \in M_{p}$. Suponlıa quc $q \in J$. Como $x \in M_{l}$, então $x_{-n_{j}} \in M_{I}$ c existe uma subscquêcia $\left\{x_{n_{k_{j}}}\right\}$ de $\left\{x_{-n_{j}}\right\}$ que couverge para $\bar{y} \in M_{q_{q}}$ tial cue $x_{-n_{k_{j}}} \rightarrow \bar{y} \in M_{q} \subset$ $\operatorname{Int}(N(J) \backslash N(\emptyset))$. Logo, cxiste $n_{k_{j_{0}}} \in \mathbb{N}$ tal que $x_{-n_{k_{j_{0}}}} \in \operatorname{Int}(N(J) \backslash N(\emptyset))$. Mas $x \in \operatorname{Inv}(\operatorname{cl}(N(I) \backslash N(J)))$. Isto gera uma contradição. Suponha que $\omega(x) \subset M_{p}$, com $p \in J$. Logo, $f^{n_{k}}(x) \rightarrow y \in M_{p} \subset \operatorname{Int}(N(J) \backslash N(\emptyset))$. Como $f^{n_{k}}(x) \in \operatorname{Inv}(\operatorname{cl}(N(I) \backslash$ $N(J))$ ), obtemos uma contradição. Portanto, $q, p \in I \backslash J$.

Agora tome $x \in M_{\bigwedge J}$. Coino $x \in \operatorname{Inv}(\operatorname{cl}(N(I) \backslash N(\theta)))$, existe una sequência $\left\{x_{n}\right\}_{-\infty}^{\infty} \subset \operatorname{cl}(N(I) \backslash N(\emptyset))$ tal que $x_{n} \in \operatorname{Inv}(\operatorname{cl}(N(I) \backslash N(\emptyset)))$, para todo $n \in \mathbb{Z}$. Supoula que $x \in N(J)$. Logo, $x \notin N(\emptyset)$, pois $(N(J), N(\emptyset))$ ć par-índice para $M_{J}$. 
Então, $f(x) \notin N(\emptyset)$, o que implica $f^{2}(x) \in N(J)$. Analogamente, $f^{n}(x) \in N(J)$ c $f^{n}(x) \notin N(\emptyset)$, para todo $n \in \mathbb{N}$. Portanto, $\omega(x) \in M_{p}$, para algum $p \in J$. O gunes ó una contradisão. Logo, $x \notin N(J)$. Istos significa (que $x \in N(I) \backslash N(J)$. Do mesmo modo unostra-se que $x_{n} \in N(I) \backslash N(J)$, prara todo $n \in \mathbb{Z}$. Resta mostrar que $\operatorname{Inv}(\mathrm{cl}(N(I) \backslash N(J))) \subset \operatorname{Int}(N(I) \backslash N(J))$. Tonic $x \in \operatorname{Inv}(\mathrm{cl}(N(I) \backslash N(J))) \subset$ $\operatorname{Inv}(\operatorname{cl}(N(I) \backslash N(\emptyset))) \subset \operatorname{Int}(N(I) \backslash N(\emptyset))$. Pelo (puc foi feito anteriormentc, temos que $x \notin N(J)$. Alénn disso, cxistc $r>0$ tal que $B(x, r) \subset N(I) \backslash N(\emptyset)$. Note que $B(x, r) \cap(N(J))^{c}$ um aberto contendo $x$, tal que $B(x, r) \cap(N(J))^{c} \subset N(I) \backslash$ $N(J)$. Portanto, $x \in \operatorname{Int}(N(I) \backslash N(J))$. E t.cunos conclnílo a verificação da primeira condição da Definição 1.9. Resta provar que $N(J)$ é positivamente invariante com rclação a $N(I)$ c que $N(J)$ é o conjunto saída.

Seja $x \in N(J)$ tal que $f(x) \in N(I)$. Como $N(\emptyset) \subset N(J)$, consideremos dois casos. O primciro ć $x \in N(\emptyset)$. Como $(N(I), N(\emptyset))$ ć par-índice para $M_{l}$, temos que $N(\emptyset)$ ć positivameutc invariante con rclação a $N(I)$. Portanto, $f(x) \in N(\emptyset)$ r $N(J)$. Sendo $(N(J), N(\emptyset))$ par-ínclice jara $M_{J}$, temos que $f(N(J) \backslash N(\emptyset)) \subset N($. Portanto, provamos que

$$
f(N(J)) \cap N(I) \subset N(J) .
$$

Oı seja, $N(J)$ é positivanınte invariante cou relaşāo a $N(I)$.

Para finalizar, seja $x \in N(I) \backslash N(J)$. Queremos mostrar que $f(x) f$ De fato, temos que $x \notin N(\emptyset)$. O resultado segue novamente do fato de qur $N(\emptyset))$ C) par-índice para $M_{l}$.

Como to caso de trio-índice, ó possível nostrar que dado una decomposiçāo cle Morse para um conjunto invariante, isolaclo, semunte existe ınra filtração índice regular associado à decomposição de Morse. Não apresentaremos a demonstração do resultado pois segue as mesmas idéias da Proposiçáo 2.11. Os detalhes desta prova podem ser cucontrados en Richeson [10].

Teorema 3.12. Seja $S$ um conjunto invariante isolado e $\mathcal{M}$ sua decomposição de Morse. Então existe $\mathcal{N}$ unn filtração indice para $\mathcal{M}$. 


\subsection{Pares de Matrizes de Conexão}

A decomposição de Morse generali\%a o conceito de decomposição atratora-repulsora para unu conjunto iuvariante isolaklo. Portant.o, mun perguntia (pue surge é qual seria o análogo do Tcorcma 2.13 para o caso de unla deconıposição de Morse. O primciro passo é "detectal" as deconıposiçōes atratoras-repulsoras de uma decomposição de Morse. O resultado a seguir aprescutia este fato. Sua demonstração se encontra ein Richesou [9].

Proposição 3.13. Seja $\mathcal{M}(\mathcal{P},<)$ uma decomposição de Morse paru o conjunto invariante isolado $S$. Se $(I, J) \in \mathcal{I}_{2}$, entĩo $M_{I . J}$ é um conjunto invariante isolado e $\left(M_{l}, M_{J}\right)$ é uma decomposição abratora-repulsora para $M_{I J}$.

Dado $I$ um intervalo, vamos construir uma dupla adjacente cspecial. Para isto, se $I \in \mathcal{I}$, defina

$$
Q_{1}:=\{q \in \mathcal{P} \backslash I \mid p \nless q \text { para todo } p \in I\} .
$$

Proposição 3.14. Sejam $\mathcal{M}(\mathcal{P},<)$ unna decomposição de Morse para o canjunto invvariante isolado $I \in \mathcal{I}(\mathcal{P},<)$ e $Q_{l}$ como untcriormcnte. Então $\left(Q_{1}, Q_{l} \cup I\right)$ é uma dupla adjacente $e Q_{l}, Q_{I} \cup I$ são intervalos atratores.

Demonstração. Temos de verificar as condições da definição de dupla adjacente. Tome $q_{1}, q_{2} \in Q_{l}$ e suponlia que $r \in \mathcal{P} \dot{c}$ tal que $q_{1}<r<q_{2}$. Suponla que existe $p_{0} \in I$ tal quc $p_{0}<r$. Logo, $\eta_{0}<q_{2}$. O que é uma contradição. Portanto, para todo $p \in I$, temos que $p \nless r$. Assinl, $r \in Q_{l}$. Isto nos mostar que $Q_{l}$ é um intervalo cm $\mathcal{P}$. É inuediato que $Q_{I} \cap I=\emptyset$.

Para verificar que $Q_{l} \cup I$ é um intcrvalo em $\mathcal{P}$, tome $q_{1}, q_{2} \in Q_{l} \cup I$. Aqui temos quatro casos a considerar:

$$
\begin{aligned}
& \text { (a) } q_{1} \text { с } q_{2} \in Q_{I}, \\
& \text { (b) } q_{1} \text { e } q_{2} \in I, \\
& \text { (c) } q_{1} \in Q_{l} \text { \& } q_{2} \in I \\
& \text { (d) } q_{1} \in I \text { e } q_{2} \in Q_{I} .
\end{aligned}
$$

Os casos (a) e (b) scguchn facilnucute do fato de cque $Q_{I}$ c $I$ são intervalos. Já caso (c) exige uma consideração cuidadosa. Suponlıa que $r \notin Q_{\iota}$. Logo, existe $p_{0} \in I$ tal 
que $\eta_{0}<r$ er< $r<q_{2}$. Eulĩ̃o, $r \in I$. Nole que o úllimo caso não ocorrc. De fato, suponlia quc cxistic $r \in \mathcal{P}$ tal que $q_{1}<r<q_{2}$. Portianto, $q_{1}<q_{2}$. Isto gera uma contradição.

É trivial ınostrar que a última condição para ser dupla adjaccute é verificada. Ou seja, $\left(Q_{1}, I\right) \in \mathcal{I}_{2}$.

Resta mostrar qur $Q_{I}$ e $Q_{I} \cup I$ são intervalos atratores. Seja $r<q$, com $q \in Q_{I}$ e suponha que $r \notin Q_{l}$. Logo, existc $p_{0} \in I$ tal que $p_{0}<r$ e $p_{0}<q$. O que ć uma contradição. Segue que $Q_{l}$ ć um intervalo atrator.

Tome $r<q$, com $q \in Q_{I} \cup I$. Temos que considerar os casos:

$$
q \in Q_{l} \text { с } q \in I
$$

Sc o primeiro caso ocorre, temos que $r \in Q_{l}$. Para o scgundo caso, temos que, se $r \in Q_{I}$, cutião estamos no caso anterior. Sc $r \notin Q_{l}$, então existe $p_{0} \in I$ tal que $p_{0}<r$ e $r<q$. Poltanto, $r \in I$.

Utilizando a Proposição 3.11 , temos o corolário a seguir.

Corolário 3.15. Sejam $\mathcal{M}$ uma decomposição de Morse para um conjunta invariante isolado $S$ e I, J intervalos em $\mathcal{I}$. Entõo $\left(N\left(Q_{I J} I J\right), N\left(Q_{1 . I} I\right), N\left(Q_{I J}\right)\right)$ é um trio-índice para o par atrator-repulsar $\left(M_{l}, M_{. l}\right)$.

As próxinas definições extrai as proprieclades algébricas que a colcção

$$
\left\{\left(C H^{*}\left(M_{l}\right), \chi^{*}\left(M_{l}\right)\right) \mid I \in \mathcal{I}\right\}
$$

possui.

Definiçäo 3.16. Seja $\mathcal{M}$ uma decamposição de Morse para um conjunto invariante isolado $S$. Uma trauça de couplexos de cocadeias com endomorfismos é uma coleção

$$
\mathcal{C}:=\{(C(I), \partial(I), c(I)) \mid I \in \mathcal{I}\}
$$

de complexos de cocadeia e endonnorfismas satisfazendo:

1. para cada $I \in \mathcal{I}$, cristem um complexo de cocadeia $C(I)$ e um endomorfismo de cocadeia $c(I): C(I) \rightarrow C(I)$ de grau zcro, 
2. para toda duplu adjacente $(I, J)$, existem aplicações $\iota:=\iota(I, I J)$ e $\rho:=$ $\rho(I J, J)$ tais que a seguinle sequência é exata

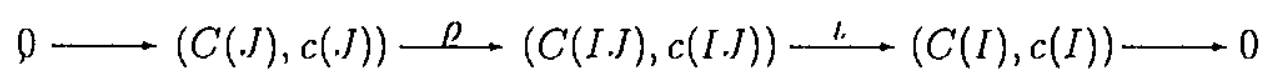

3. para todo trio adjacente $\left(I, J, K^{*}\right)$ a seguinte trança de diagramas é comutativa.

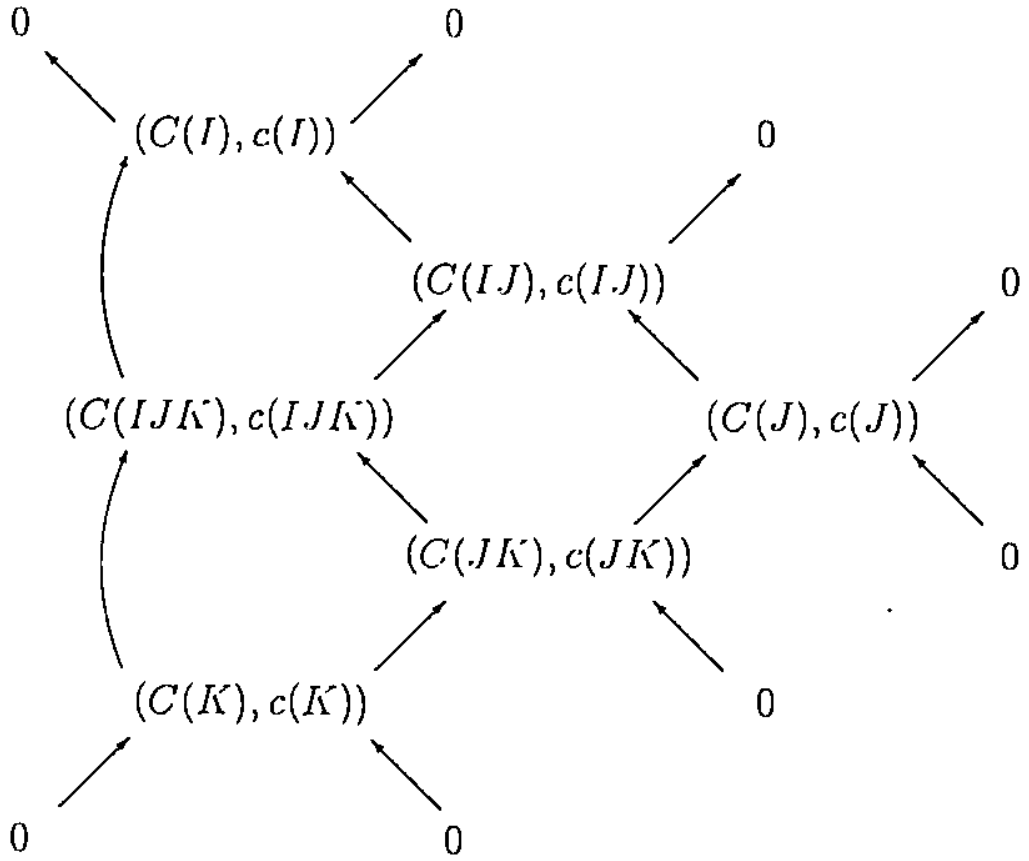

Definição 3.17. Seja $\mathcal{M}$ uma decomposição de Morse para um conjunto invariante isolado $S$. Uma trança de módulos graduados com endomorfismos é uma coleçâo $\mathcal{H}:=\{(H(I), a(I)) \mid I \in \mathcal{I})\}$ de módulos graduados e endomorfismos lineares tal que

1. paru cada $I \in \mathcal{I}$, existern um módulo gradnado $H(I)$ e um cndomorfismo linear $a(I): H(I) \rightarrow H(I)$ de grau zero,

2. para toda dupla ndjacente $(I, J)$, existe uma sequência exata longo de módulos e endomorfismos

$$
\cdots \stackrel{\delta^{n-1}}{\longrightarrow}\left(H^{n}(J), a^{n}(J)\right) \stackrel{\rho^{n}}{\longrightarrow}\left(H^{n}(I J), a^{n}(I J)\right) \stackrel{\iota^{n}}{\longrightarrow}\left(H^{n}(I), a^{n}(I)\right) \stackrel{\delta^{n}}{\longrightarrow} \cdots,
$$

3. para todo trio adjacente $\left(I, J, K^{\prime}\right)$ a scyuinte trança de diagramas é comutativa. 


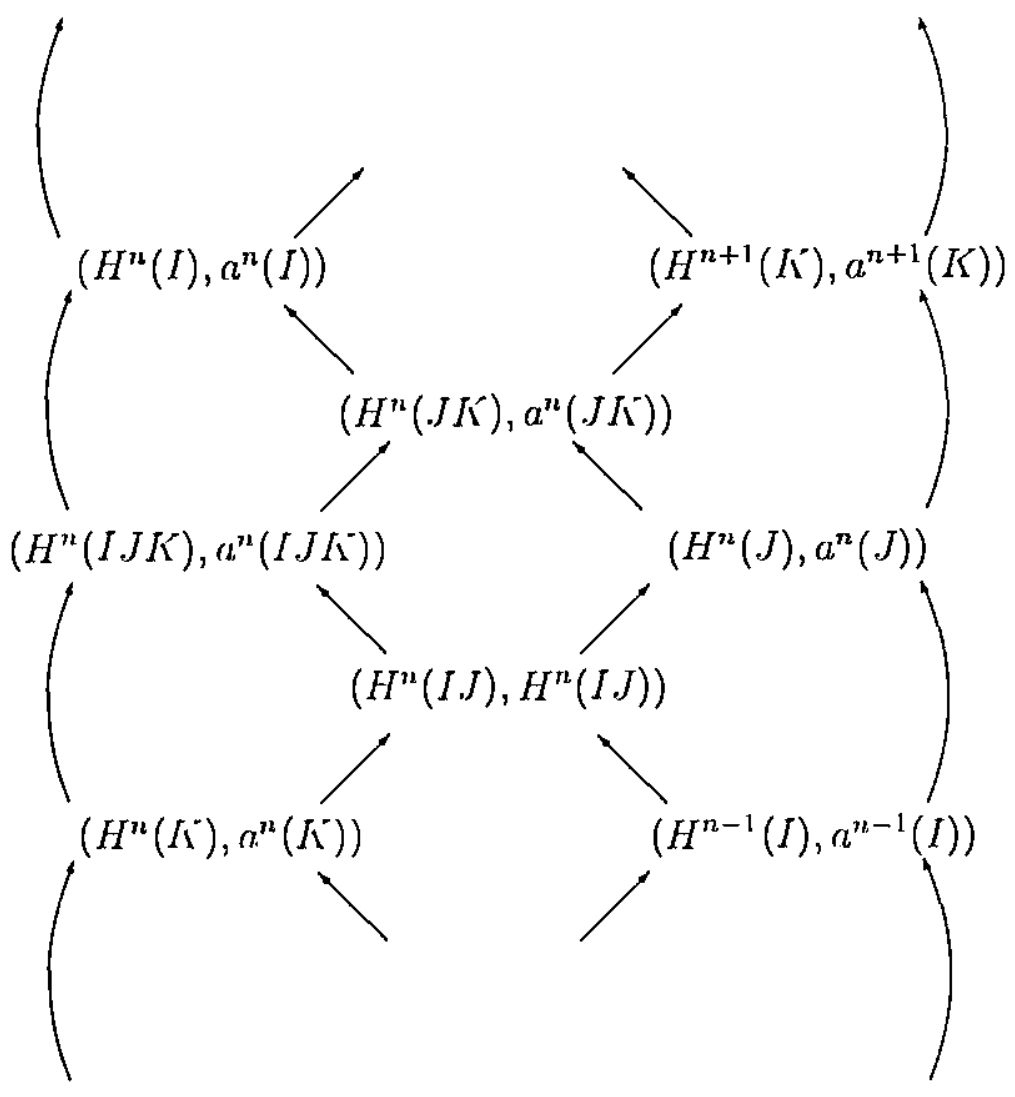

É imediato que

Proposição 3.18. Seja $\mathcal{C}$ uma trança de complexos de cocadeias com endomorfismos. Passando a.o nível de cohomologia oblemos nma trnnça de módulos graduados e endomorfismas, que denolamos por $\mathcal{H C}$.

Proposição 3.19. Seja $S$ um conjunto invarianle isolado e $\mathcal{M}(\mathcal{P},<)$ sua decamposiçäo Morse. Definimos

$$
\mathcal{C}(\mathcal{M}):=\left\{\left(\mathcal{L} C\left(M_{1}\right), \mathcal{L} f\left(M_{l}\right)\right) \mid I \in \mathcal{I}\right\}
$$

$e$

$$
\mathcal{H}(\mathcal{M}):=\left\{\left(C H^{*}\left(M_{l}\right), \chi^{*}\left(M_{I}\right)\right) \mid I \in \mathcal{I}\right\}
$$

Então $\mathcal{C}(\mathcal{M})$ é uma tranıça de conıplexos de cocadeias e endomorfismos e $\mathcal{H}(M)$ é uma trança de módulos graduados $c$ endomorfismos.

Demonstração. Pelo Corolário 3.15, daclos $I, J$ intervalos $\mathrm{cm} \mathcal{I}$, temos que $(N(Q), N(Q I), N(Q I J)),(N(Q), N(Q I), N(Q I J K)),(N(Q), N(Q I J), N(Q I J K))$ 
e $(N(Q I), N(Q I J), N(Q I J K))$ são trios-índices, onde $Q_{I J K}=Q$. Logo, pela Proposição 2.13 , temos a seguinte sequência exata curta

$$
0 \rightarrow\left(\mathcal{L} C^{*}\left(M_{J}\right), \mathcal{L} f\left(M_{, J}\right)\right) \stackrel{p^{\#}}{\longrightarrow}\left(\mathcal{L} C^{*}\left(M_{l, I}\right), \mathcal{L} \int\left(M_{l, I}\right)\right) \stackrel{\iota^{\#}}{\longrightarrow}\left(\mathcal{L} C^{*}\left(M_{I}\right), \mathcal{L} f\left(M_{I}\right)\right) \rightarrow 0 .
$$

Passando ao nível de cohomologia, obtemos o sequência exat,a longa de espaços vetoriais e autoonorfismos.

$$
\ldots \stackrel{\delta^{\eta-1}}{\longrightarrow} \operatorname{Con}^{q}\left(M_{, J}\right)^{\rho^{q}} \operatorname{Con}^{q}\left(M_{, J}\right) \stackrel{\iota^{\prime}}{\longrightarrow} \operatorname{Conl}^{q}\left(M_{l}\right) \stackrel{\delta^{q}}{\longrightarrow} \operatorname{Con}^{q+1}\left(M_{J}\right) \stackrel{\rho^{q+1}}{\longrightarrow} \cdots
$$

É fácil ver que $\mathcal{C}(\mathcal{M})$ e $\mathcal{H}(\mathcal{M})$, cono definidos no enunciado da proposição satisfazcm as condições das Definiçõcs 3.16 e 3.17 , respectivamente.

A seguir definimos o conceito de homomorfisıno cntre duas tranças de módulos.

Definição 3.20. Sejam $\mathcal{M}$ uma decomposiçă de Morse para um conjunto inuariante isolado $S, I$ intervalo em $\mathcal{I}$ e $\mathcal{H}(P)=\{(I I(I), a(I)) \mid I \in \mathcal{I}\}, \mathcal{H}^{\prime}(P)=$ $\left\{\left(H^{\prime}(I), a^{\prime}(I)\right) \mid I \in \mathcal{I}\right\}$ duass trunças de módiulos graduados $c$ endornorfismos. Um homomorfismo entre tranças de módulos graduados, $\Psi: \mathcal{H} \rightarrow \mathcal{H}^{\prime}$, é uma coleção de homomorfismos $\psi(I): H(I) \rightarrow H^{\prime}(I)$, definido parn todo interaulo $I \in \mathcal{I}$, tal que para toda dupla adjacente $(I, J)$ o diagrama

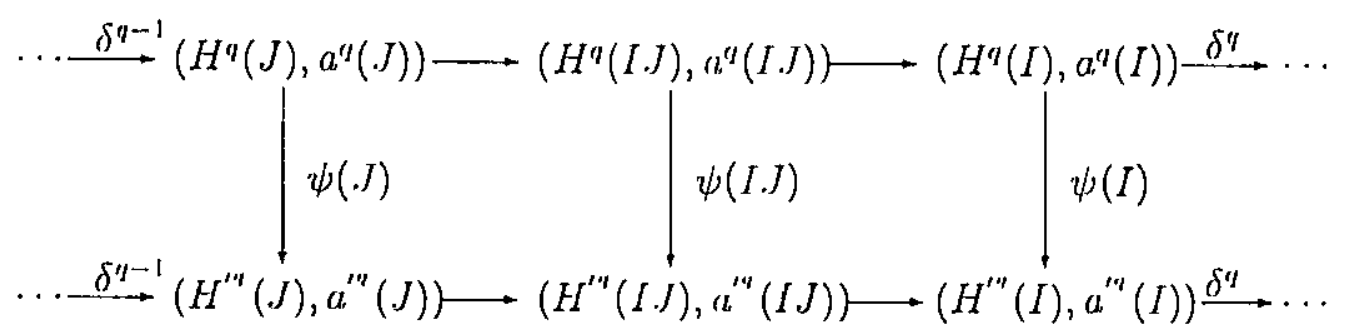

é comutativo.

Observação 3.21. Sc para cada $I \in \mathcal{I}$, da defiluição anterior, $\psi(I)$ for um isomorfismo, cntão clizemos çuc $\Psi$ ć um isonorfismo.

O resultado enunciado a seguir apresenta uma condição para que $\Psi$ seja isomorfismo.

Proposição 3.22. Supontıa que $\Psi: \mathcal{G}^{\prime} \rightarrow \mathcal{G}$ seja um homomorfismo de tranças de módulas graduados tal que $\psi(p)$ seja isomorfismo para todo $p \in \mathcal{P}$. Entäo $\Psi$ é um isomorfismo. 
Demonstração. Vamos usar indução sobre o número cle elcmentos de $I \in \mathcal{I}$.

Suponlia que $I=\{p\}$. Logo, por hipólese $\psi(p)$ ć un isomorlismo. Suponha que o resultado seja válido para todo intcrvalo $I$ com $m-1$ elementos. Mostremos que o resultado é válido para un intervalo $I$ com $m$ i clenentos.

Se existe $\bar{p} \in I$ tal que $\bar{p} \nless q$ para todo $q \in I$, clefma $\tilde{K}=I \backslash\{\bar{p}\}$. Neste caso ć fácil verificar que $(\tilde{K}, \bar{I})$, onde $\vec{I}=\{\bar{p}\}$, ć mua dupla adjacente. Caso contrário, isto ć, sc para cada $p \in I$ scmpre existe $q_{p} \in I$ tal que $p<q_{p}$ ou $q_{p}<p$. Fixe $p_{0} \in I$. Scm perda de generalidade podemos supor que $\eta_{0}<q_{p_{0}}$. Defina

$$
K_{p_{0}}:=\left\{q \in I \mid p_{0}<q\right\}
$$

Notc que $K_{p_{0}} \neq \emptyset$ с $I=\left(I \backslash K_{p_{0}}\right) \cup K_{p_{0}}$. Agrora tome $r_{1}, r_{2} \in K_{p_{0}}$ e suponha que existe $r \in I$ tal (ue) $r_{1}<r<r_{2}$. Logo, $p_{0}<r_{1}<r<r_{2}$. Isto implica que $r \in K_{p_{0}}$, ou seja, que $K_{p_{0}} \dot{c}$ un intcrvalo. De modo anćlogo mostra-se que $I \backslash K_{p_{0}}$ é um intervalo. É claro que $\left(I \backslash K_{p_{0}}\right) \cap K_{p_{0}}=\emptyset$ c se $p \in K_{p_{0}}$ então $p \nless q$, para todo $q \in\left(I \backslash K_{p_{0}}\right)$. Fazendo $\tilde{K}=\left(I \backslash K_{p_{0}}\right)$ c $\bar{I}=K_{p_{0}}$, novamente temos que $(\tilde{K}, \bar{I})$ é uma dupla adjacente. Consideraurlo o diagrama apresentado na Definição 3.20, obtemos o cliagrama

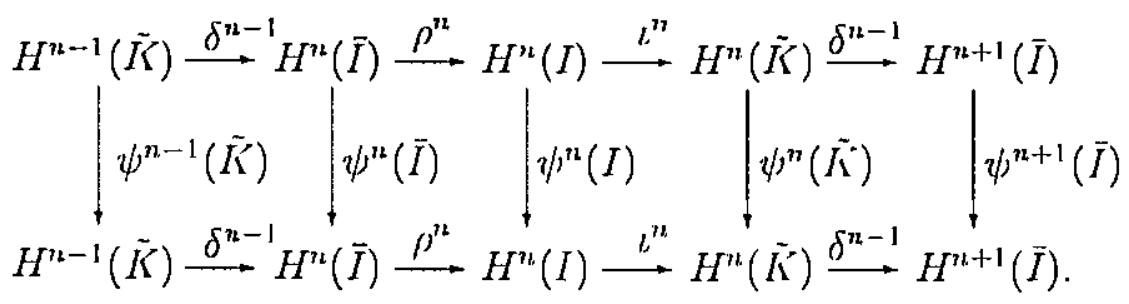

Pela hipótese dle indução temos que $\psi(\tilde{K})$ e $\psi(\bar{I})$ são isomorfismos. Aplicando o Lema alos Cinco, obtemos cute $\psi(I)$ i um isomorfismo.

A seguir aprescntaınos os preliminares para a introdução do conceito de par de matrizes de concxão.

Definição 3.23. Seja $\mathcal{M}(\mathcal{P},<)$ uma decomposição de Morse para um conjunto invariante isolado $S$. Dado $I \in \mathcal{I}$, definimos $C \Delta(I):=\bigoplus_{p \in l} C H^{*}\left(M_{p}\right)$.

Scja $\Delta: C \Delta(\mathcal{P}) \rightarrow C \Delta(\mathcal{P})$ una alplicaçĩo lincar. 
Observaçāo 3.24. A aplicação $\Delta$ pode scr vista na forma de uma matriz $|\mathcal{P}| \times|\mathcal{P}|$,

$$
\Delta=\left(\Delta_{p q}\right)_{p, l \in \mathcal{P}}
$$

onde $\Delta_{p q}: C H^{*}\left(M_{q}\right) \rightarrow C H^{*}\left(M_{p}\right)$ ć tamuló́m mma aplicação linear.

Definição 3.25. Sejam $\mathcal{M}(\mathcal{P},<)$ uma decomposição de Marse para a conjunto invariante isolado $S$ e $\Delta: C \Delta(\mathcal{P}) \rightarrow C \Delta(\mathcal{P})$ a aplicação linear. Se $p<q$ implicar $\Delta_{p q}=0$, diremos que esla é umno matrì triangular inferior. Mas, se $p \leq q$ implicar $\Delta_{l m}=0$, dizemas que $\Delta e ́$ uma matrì triangular estritantente infcrior. Se $\Delta$ for de gran uru e $\Delta^{2}=0, \Delta$ é chamada opcrarlor cobordo.

Observação 3.26. Note que para cacla $I \in \mathcal{I}$, podemos definir a aplicação

$$
\Delta(I):=\left(\Delta_{m, q}\right)_{p, q \in l}: C \Delta(I) \rightarrow C \Delta(I) .
$$

O resultado a seguir nos aprescontia concliçōes sobrc a aplicação $\Delta$ para que $(C(I), \Delta(I))$ seja um conplcxo dc cocacleia.

Lema 3.27. Seja $\mathcal{M}(\mathcal{P},<)$ uma ilecompasição de Marse para o conjunto invariante isalada $S$. Se $\Delta$ é um opcrador coborclo triangular estritamente inferior, então, para cada $I \in \mathcal{I}, \Delta(I)$ também a é. Em parlicular, $(C(I), \Delta(I))$ é um camplexo de cocadeia.

DemonstraçÃo. Scja $I \in \mathcal{I}$. Para mostrar que $\Delta(I)$ é triangular cstritamente infcrior, basta lcmbrar que se $p<q$ cm $I$, entr̃o $p<q$ em $\mathcal{P}$. O que inplica $\Delta_{p q}=0$.

Resta mostrar que $(\Delta(I))^{2}=0$. Para tanto, basta lenibrar que $(\Delta(I))^{2}$ é o produto das matrizas $\left(\Delta_{p q}\right)_{p, q \in l}$.

Scjann $\mathcal{M}(\mathcal{P},<)$ uma decomposiçã̆o do Morse para o conjunto invariante isolado $S$ c $(I, J)$ uma dupla acljacente. Assim faz scntido pensar nas aplicações $\iota: M_{I} \rightarrow$ $M_{I J}$ inclusão canônica c $\rho: M_{J, f} \rightarrow M_{J}$, projcção canônica, c assim, obtcmos as seguintes aplicaçōes indnzidas em nível de colıomologia

$$
i^{*}: C \Delta(I J) \rightarrow C \Delta(I) \mathrm{c} \rho^{*}: C \Delta(J) \rightarrow C \Delta(I J)
$$

Do mesmo morlo quc fizemos anteriormente, dado $d: C \Delta(\mathcal{P}) \rightarrow C \Delta(\mathcal{P})$ uma aplicação de cocadleia que seja lincar, triangular inforior de grau zero, podemos cscrcvê-la como $11 \mathrm{ma}$ matriz

$$
d=\left(d_{m, q}\right)_{p, q \in P}
$$


onde $d_{p q}: C H^{*}\left(M_{q}\right) \rightarrow C H^{*}\left(M_{p}\right)$. E, novantente, para cada $I \in \mathcal{I}$, podemos definir

$$
d(I)=\left(l_{m, q}\right)_{n, q \in l}: C \Delta(I) \rightarrow C \Delta(I)
$$

Proposição 3.28. Seja $\mathcal{M}(\mathcal{P},<)$ uma decomposição de Morse para o conjunto invariante isolndo $S$. Sejann $\Delta: C \Delta(\mathcal{P}) \rightarrow C \Delta(\mathcal{P})$ um operador cobordo triangular estritamente inferior $e d: C \Delta(\mathcal{P}) \rightarrow C \Delta(\mathcal{P})$ umno aplicação linear de cocadeia triangular inferior de grau zero. Então

$$
\mathcal{C} \Delta(\mathcal{M}):=\{(C(I), \Delta(I), d(I)), \mid I \in \mathcal{I}\}
$$

é uma trança de complexas de cocadeia e endomorfismos. Passando ao nível de cohomologia oblenus uma trnnuşa de módulos gradnados e endomorfismas que será denotado por $\mathcal{H} \Delta(\mathcal{M})$.

Finalmente, estamos em condições de definir o par de matrizes de conexão para unlıa decomposição de Morse.

Definição 3.29. Seja $\mathcal{M}(\mathcal{P},<)$ unua decomposiçăo de Marse para o conjunto invariante isolado $S$. Sejam $\Delta: C \Delta(\mathcal{P}) \rightarrow C \Delta(\mathcal{P})$ um operollor cobordo triangular estritamente inferior e $d: C \Delta(\mathcal{P}) \rightarrow C \Delta(\mathcal{P})$ umo aplicação linear de cocadeia triangular inferiar de grau zero. O par de matrizes $(\Delta, d)$ é chamada par de matrizes de conexão para a decomposição de Morse $\mathcal{M}(\mathcal{P},<)$ se a trança de módulos graduados e endomorfismos $\mathcal{H} \Delta(\mathcal{M})$ é isamorfa a trança de módulos graduados $e$ endomorfismos $\mathcal{H}(\mathcal{M})$.

Observação 3.30. Recorde quc $\mathcal{H}(\mathcal{M})$ foi clctinido na Proposição 3.19 . 


\section{Capítulo 4}

\section{De volta à Decomposição Atratora-repulsora}

Ao fillal clo Capítulo 3, somos levalos a perguntar sobre a existência de um par de matrizes de conexão para una clccomposição de Morse de um conjunto invariantc isolado. Neste capítulo aprescutianıs a construção de una par de matrizes de concxão para una decomposição attratura-repulsora. $\mathrm{Na}$ Seção 4.1, essa construção ó clescrita. Encerranos este: traballıo listanclo algumas consequuências do resultado principal deste capítulo, Tooroma 4.2, e de resultados de capítnlos anteriores cujo objctivo é ilustrar como o Índicc de Conlcy, atravćs do par de matrizes de conexão, resulta con alguma infornação a resspeito da dinânicia.

\subsection{Pares de Matrizes de Conexão para o Par Atrator-Repulsor}

En [10], Richeson prova a existência de un par de inatrizcs cle conexão para uına dada deconn esiçàa de Morse. Sua demonstração ć de carater purannente algébrico, utilizindo resulatarlos de: Álgebra Linear e: Ifomologia. Apresentamos aqui a demonstração para o caso de mua decomposição atratora-repulsora.

Como anteriomentce, a colımologia dos índices de Conley serão calculados usando coeficicutes sobre un corpo.

Definição 4.1. Scjam $S$ um conjunto invariante isolado e $(A, R)$ sua decomposição 
atratora-repulsorc. Scja $D^{q}=C H^{\prime}(A) \times C H^{q}(R)$ e considere a aplicaçäo linear

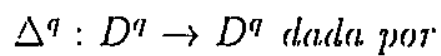

$$
\Delta^{\prime}=\left(\begin{array}{cc}
0 & 0 \\
\delta^{\prime} & 0
\end{array}\right)
$$

onde $\delta^{\prime}$ é a aplicação cobordo aprescutalda pela sequência exata longa da Proposição 2.13 .

O resultado a seguir mostra que a aplicação lincar $\Delta^{q}$ definida anteriormente é de fato o operarlor coborde ra Definiçno 3.20 e apresentia a aplicação linear $d$ que faz com que $(\Delta, l)$ seja mu par cle mallizes de concxão para $S$.

Teorema 4.2. Sejan $(D, \Delta)$ como na definição anblerior. Então $(D, \Delta)$ é um complexo de cocadeia com $H^{q}(D) \cong C H^{q}(S)$. Além disso, existe uma aplicação de cocadcia $d: D \rightarrow D$ tal que sua induzida em nivel de colomologia, $d^{*}$, é conjugada o. $\lambda^{*}(S)$.

Demonstrą̧Ão. Princiramenté mostremos que $(D, \Delta)$ é um complexo de cocadcia. Note que

$$
\Delta^{q-1} \circ \Delta^{q}=\left(\begin{array}{cc}
0 & 0 \\
\delta^{q-1} & 0
\end{array}\right)\left(\begin{array}{cc}
0 & 0 \\
\delta^{q} & 0
\end{array}\right)=\left(\begin{array}{ll}
0 & 0 \\
0 & 0
\end{array}\right) .
$$

Logo, $\Delta$ ć um operador cobordo. Temos que,

$$
H^{\prime \prime}(D)=\frac{\operatorname{licr} \Delta^{\prime}}{\operatorname{Im} \Delta^{\prime-1}}
$$

Mas $\operatorname{ker} \Delta^{q} \cong \operatorname{licr} \delta^{q} \times C H^{q}(R)$ c Im $\Delta^{q-1} \cong \operatorname{Im} \delta^{q-1}$. Portanto,

$$
H^{q}(D) \cong \frac{\operatorname{kn} \delta^{q} \times C H^{q}(R .)}{\operatorname{In} \Delta^{q-1}}
$$

Verifica-sc facilnente que

$$
\frac{\text { ker } \delta^{q} \times C H^{q}(R)}{\operatorname{In} \Delta^{q-1}} \text {; isomorfo a ker } \delta^{q} \times \frac{C H^{q}(R)}{\operatorname{In} \delta^{q-1}} \text {. }
$$

Pelo Tcorema 2.13, temos que $\operatorname{Im} \rho^{q} \subset C H^{\prime \prime}(S)$. Assim faz scntido fazer considerar o cspaço quociente $C H^{q}(S) /$ lu $\rho^{\prime}$. É lácil ver que

$$
\frac{C H^{\prime \prime}(S)}{\operatorname{In} \iota^{\prime \prime}} \text { cisomorfo a } \operatorname{Im} \iota^{\prime} \text {. }
$$


Logo,

$$
C I I^{q}(S) \cong \operatorname{Im} \rho^{q} \times \operatorname{Im} \iota^{q} .
$$

Pelo Primeiro Teorema do Isomortismo, temos gue

$$
\operatorname{In} \rho^{q} \cong \frac{C H^{\prime}(R)}{\operatorname{ker} \rho^{\eta}}
$$

Como a sequência do Teorena 2.13 é exatian, segue que

$$
\operatorname{Ln} \rho^{q} \cong \frac{C H^{q}(R)}{\operatorname{In} \delta^{q-1}} \circ \operatorname{In} \iota^{q}=\operatorname{ker} \delta^{q}
$$

Logo,

$$
C H^{q}(S) \cong \frac{C H^{q}(R)}{\operatorname{In} \delta^{q-1}} \times \operatorname{kcr} \delta^{q} \cong H^{q}(D) .
$$

E concluímos a primeira parto do teorenua. Resta mostrar que cxiste unla aplicação d. $D \rightarrow D$ tal que sna induricla $d^{*}$ o conjugarla ì $\chi^{*}(S)$.

Como $C H^{q}(S)$ á isomorfo a ker $\delta^{q} \times \frac{C H^{q}(R)}{\operatorname{lm} \delta q-1}$, wanos que $\chi^{*}(S)$ co conjungada ì mua aplicação da forma

$$
\left(\begin{array}{cc}
\frac{r^{\prime}}{d l_{k, 1}^{q}} & 0 \\
u^{q}
\end{array}\right)
$$

onde $a^{\prime \prime}$ ć a restrição de $\chi^{q}(A)$ a krr $\delta^{q} \mathrm{e} a^{q}$ ć a aplicação sobre inchuzida por $\chi^{q}(R)$. O próximo passo é encoutrar $l_{R, 1}^{\prime \prime}: C H^{q}(A) \rightarrow C H^{\prime \prime}(R)$ tal que induz $\overline{l_{R A}^{\prime \prime}}$. Completauclo una hase de ker $\delta^{q}$ para mua base de: $C H^{q}(A)$ o utilizaudo $\overline{l_{R A}^{q}}$, um argumento simples cle Álgebra Linear permite comstruir una aplicação linear com a propricdade descejacla.

Defina $d^{q}: D^{q}=C H^{q}(.4) \times C H^{q}(R) \rightarrow C H^{q}(A) \times C H^{q}(R)$ por

$$
\left(\begin{array}{cc}
\chi^{q}(A) & 0 \\
d_{R, 1}^{q} & \chi^{\prime \prime}(R)
\end{array}\right)
$$

Tambén é simples demonstrar (puc d é uma aplicação de cocadeia, isto é,

$$
d^{\eta+1} \circ \Delta^{q}=\Delta^{q} \circ d^{q} \text {. }
$$

Alóm clisso, $d^{*}$ é conjugarla à $\chi^{*}(S)$. 


\subsection{Detectando Órbitas de Conexão}

Como o par de matrizes de concxĩo implicar a existência de órbitas de concxão? Usando o Teorena 4.2 c o Teorema 2.13 , encerramos cste trabalho com algumas situações cm que ć possível concluir a cxistência de órbitas de conexão e oncle não c.

Corolário 4.3. Sejam $S$ um conjunto invuriunte isolado e $(A, R)$ sua decomposição atratora-repulsora. Se existe um nuirncro natural $n$ tal que

$$
\operatorname{Con}^{\prime}(A)= \begin{cases}\left(C H^{\prime \prime}(A), \chi^{q}(A)\right), & q=n \\ 0, & q \neq n\end{cases}
$$

$e$

$$
\operatorname{Con}^{q}(R)= \begin{cases}\left(C H^{q}(R), x^{q}(R)\right), & q=n, \\ 0, & q \neq n,\end{cases}
$$

cutão $\delta^{\eta}=0$, para todo $q \in \mathbb{Z}, C H^{\eta}(S)=0$, se $q \neq n$ e $C H^{n}(S) \cong C H^{n}(A) \times$ $C^{\prime} H^{n}(R$.$) .$

Demonstra̧̧ão. En vista clas lịpóteses, a sequência exata longa da Proposição 2.13 toma-se

$$
0 \stackrel{\delta^{n-1}}{\longrightarrow} C H^{n}(R) \stackrel{b^{n-1}}{\longrightarrow} C H H^{n}(S) \stackrel{\rho^{n}}{\longrightarrow} C H^{n}(A) \stackrel{\delta^{n}}{\longrightarrow} C H^{n+1}(R)=0
$$

(:

$$
C H^{n+1}(R)=0 \stackrel{\rho^{n+1}}{\longrightarrow} C H^{n+1}(S) \stackrel{\iota^{n+1}}{\longrightarrow} C H^{n+1}(A)=0 \stackrel{\delta^{n+1}}{\longrightarrow} 0 .
$$

A exatidão das sequênucias anteriores implica que $\operatorname{Im} \iota^{n+1}=\operatorname{ker} \delta^{n+1}$. Mas $\delta^{n+1}=$ 0. Portanto, $\operatorname{Im} \iota^{n+1}=0$. Por ontro laxlo, $\operatorname{lec} \iota^{n+1}=\operatorname{Im} \rho^{n+1}$. Como $\operatorname{Im} \rho^{n+1}=0$, temos (1ue $\operatorname{Im} \iota^{n+1}=0$ e $\operatorname{kec} \iota^{n+1}=0$. Senclo $\operatorname{dim} C I I^{n+1}(S)=\operatorname{dim} \operatorname{Im} \iota^{n+1}+$ dimker $t^{n+1}$, temos que $\operatorname{dim} C H^{n+1}(S)=0$. Mas,

$$
C H^{n}(S) \cong \operatorname{ker} \delta^{n} \times \frac{C H^{n}(R)}{\operatorname{Im} \delta^{n-1}} \cong C H^{n}(A) \times C H^{n}(R),
$$

pois Im $\delta^{n-1}=0$. Para $q \neq n, n+1$, ć imediatio ver que $\delta^{q}=0$ e $C H^{4}(S)=0$.

Corolário 4.4. Sejam $S$ umu conjunto invariante isolado e $(A, R)$ uma decomposicão atralora-repulsora para $S$. Suponha que

$$
\operatorname{Colr}^{q}(A)= \begin{cases}(\mathbb{Q}, \mid l d), & q=1 \\ 0, & q \neq 1\end{cases}
$$


e

$$
\operatorname{Cou}^{\prime \prime}(R)= \begin{cases}(\mathbb{Q}, \mathrm{Icl}), & q=1 \\ 0, & q \neq 1 .\end{cases}
$$

Se a. aplicação dl $l_{R A}$ é nüo nullu, conlào $\mathrm{C}(R, A ; S) \neq \emptyset$.

Demonstração. Pelo Corolátio $4.3, \delta^{q}=0$, para todo $q \in \mathbb{Z}, C H^{q}(S)=0$, para todo $q \neq 1$ e $C H^{1}(S)=\mathbb{Q} \times \mathbb{Q}$. Pelo Teorema $4.2, \chi^{*}(S)$ é conjugada à

$$
\left(\begin{array}{cc}
\mathrm{Idl} & 0 \\
l_{R, \lambda} & \mathrm{Id}
\end{array}\right)
$$

onde dlRA $: C H^{1}(R.) \rightarrow C H^{1}(A)$. Como $\operatorname{dim} C H^{1}(R)=\operatorname{dim} C H^{1}(A)=1$ e d $d_{R A} \neq 0$, temos culc

$$
\left(\begin{array}{cc}
\operatorname{Id} & 0 \\
d_{R, 4} & \mathrm{Id}
\end{array}\right)
$$

nũo ć conjughadia a

$$
\left(\begin{array}{cc}
\mathrm{Id} & 0 \\
0 & \mathrm{Id}
\end{array}\right)
$$

Note cue csta ć a apresculação para $\chi^{\prime}(R) \times \lambda^{\prime}(A)$. O resultado segne do Corolário 2.15 .

Observaçāo 4.5. So $d_{R A}=0110$ corolário antcerior, não tcmos garantias que $S=$ $A \cup R$.

A conchusào do corolário anterior foi possível deviclo a especial escolha dos índices

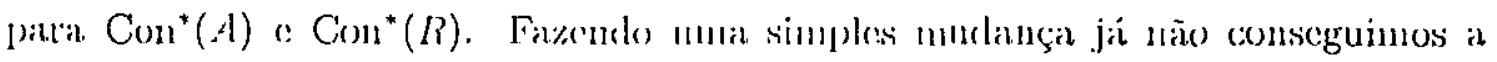
mesmar conclusão. É a (pue veu a nos mostrar o exemplo a seguir.

Exemplo 4.6. Scjan $S$ uu conjunto invariauı isolado e $(A, R)$ a decomposição alratora-repulsora para $S$ tal que

$$
\operatorname{Con}^{q}(A)= \begin{cases}(\mathbb{Q}, \operatorname{Ir}), & \text { sis } q=1, \\ 0, & \text { caso contrário }\end{cases}
$$

c

$$
\operatorname{Con}^{q}(R)= \begin{cases}(\mathbb{Q},-\operatorname{Irl}), & \text { sc } q=1, \\ 0, & \text { (aso contrairio. }\end{cases}
$$

Procedendo como anteriormente, $\chi^{*}(S)$ será conjugada à

$$
\left(\begin{array}{ll}
\mathrm{Id} & 0 \\
d_{R A 1} & -\mathrm{Il}
\end{array}\right)
$$




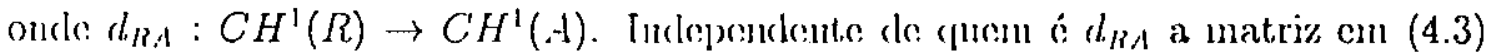
semupre co coujugadia à $\lambda^{*}(A) \times \lambda^{*}(R)$.

É fácil construir excmplos oncle os ínclices de $A$ e $R$. são cono $\mathrm{cm}$ (4.1) e (4.2), respectivannente e $\mathrm{C}(R, A ; S) \neq \emptyset$; c exemplos em que $\operatorname{Con}^{*}(A), \operatorname{Con}^{*}(R)$ sào novamente conno acima, mass $S=. A \cup R$.

No próximo resultaclo $\sigma(A)$ representa o conjunto clos autovalores de unna aplicaçào lincar $A$.

Corolário 4.7. Sejam $S$ um conjunto invariante isplado e $(A, R)$ sua decomposição alratorth-rcpulsora. Suponlin que para alyuin urm utumero natural $n$

$$
\operatorname{Con}^{\prime \prime}(A)= \begin{cases}\left(C H^{\prime}(A), \chi^{q}(A)\right), & q=n, \\ 0, & q \neq n,\end{cases}
$$

c.

$$
\operatorname{Con}^{\prime \prime}(R)= \begin{cases}\left(C H^{\prime}(R), x^{\prime \prime}(R)\right), & q=n, \\ 0, & q \neq n .\end{cases}
$$

Se $\sigma\left(\chi^{q}(S)\right)$ não está continlo ua união disjunta de $\sigma\left(\chi^{q}(A)\right)$ com $\sigma\left(\chi^{q}(R)\right)$, então $\mathrm{C}(R, A ; S) \neq \emptyset$.

DemonstraçÃo. Nesta condiçōes $\chi^{q}(S)$ não ć conjugada a $\chi^{q}(A) \times \chi^{q}(R)$ e o resultado sogue do Corolário 2.15.

O resultado a seguir foi provado por Reineck [11] para o caso de fluxos contínuos. Apresentianos a provin para o (anso do sistemas dinâunicos discretos.

Corolário 4.8. Sejam $\mathcal{M}(\mathcal{P},<)$ uma decomposição de Morse para um conjunta invariante isolado, $e(\Delta, l)$ um par rle mutrizes rle conexão. Suponha $p, q \in \mathcal{P}$ tais que $p<q$ q $\{p, q\}$ scja um internolo. Snponlıa ainda que a aplicação $\triangle_{p q}$ : $C H^{*}\left(M_{q}\right) \rightarrow C H^{*}\left(M_{p}\right)$ seja não nula. Entũa existe conexão entre $M_{q}$ e $M_{p}$.

DemonstraçÃo. Notc que a aplicação $\Delta(\{p, q\})$ ć clada pela matri\%

$$
\left(\begin{array}{cc}
0 & 0 \\
\Delta_{p q} & 0
\end{array}\right)
$$

onde $\Delta_{p l l}: C H^{*}\left(M_{q}\right) \rightarrow C H^{*}\left(M_{p}\right)$ c uma a plicação não uula. Verifica-se facilinente (que ker $\Delta(\{p, q\}) \cong C H^{*}\left(M_{p}\right)$ e In $\Delta(\{p, q\}) \cong C H^{*}\left(M_{p}\right)$. Logo, $H^{*}(C \Delta(\{p, q\})) \cong$ 
0. Como $(\Delta, d)$ o um par de matrizes de concxĩo, entiào

$$
C H^{*}\left(M_{\{p, q\}}\right) \cong 0
$$

Mass se $M_{p}=\emptyset$ on $M_{q}=\emptyset$, então $\Delta_{p q}=0$. Portanto, $M_{p} \neq \emptyset$ с $M_{q} \neq \emptyset$. Supouha que $M_{\{p, q\}}=M_{p} \cup M_{q}$. Notiando que esta união ć disjunta, usando o Corolário 2.14 juntibuente com o 'Teorema 4.2, temos (jue $\Delta(\{p, q\})=0$. O que é unta contradição. Logo, $\mathrm{C}\left(M_{p}, M_{q}, M_{\{p, q\}}\right) \neq \emptyset$. 


\section{Referências Bibliográficas}

[1] T. Arnold, C. Jones, K. Mischaikow, G. Raungel, Dyanamical Systems Montecatini Terme 1994, R. Johnson, ed., Lect. Notes Math. 1609, Springer 1995.

[2] C. Conley, Isolated Invariant, Sets and the Morse Indcr, CBMS Reg. Conf. Ser. in Math., 38, AMS, Proviclence, 1978.

[3] A. Dold, Leclures on Algebraic Topology, Springer, Berlin, 1972.

[4] J. Franks c D. Richeson, Slift e(puivalence and the Conley index, Trans. AMS, a aparecer.

[5] R. Franzosa, The connection matrix theory for Morse decompositions, Trans. AMS 311, (1989) 561-592.

[6] M. Mrozek, Lcray functor and colomological Conley index for discrete dynamical systems, Trans. AMS, 318 (1990) 149-178.

[7] .J. Muukres, Elements of Alyebrnic: Topology, Acldison-Wesley, 1984.

[8] .J. Palis e W. de Melo, Introduçãn aos Sistemas Dinânuicos, Projeto Euclides, 1978.

[9] D. Ricluson, Conmectimn Mntrix Pairs for the Discrete Conley Index, tese de doutoraclo, Northwesten University, 1998.

[10] D. Richeson, Comnection matrix pairs for the cliscrete Conley index, preprint.

[11] J. Reineck, The comnection matrix in Mense-Smale Hows, Trans. AMS, 322 (1990) 523-544.

[12] K. P. Rybakowski, The Homatopy Inder and Partial Diffcrential Equations, Universitext, Springer-Verlag, 1987. 
[13] E. Spanier, Algebraic Topology, McGraw-Hill, 1966. 